\title{
The Tamagawa number conjecture for CM elliptic curves
}

\author{
by Guido Kings
}

Preprint March 17, 2000

\section{Introduction}

In this paper we prove the Tamagawa number conjecture of Bloch-Kato for CM elliptic curves using a new explicit description of the specialization of the elliptic polylogarithm.

The Tamagawa number conjecture describes the special values of the Lfunction of a variety in terms of the regulator maps of the K-theory of the variety into Deligne and étale cohomology (see 1.1 for the exact formulation). There are only two cases proven so far in the non critical situation, both due to Bloch and Kato [Bl-Ka]: The first is the Riemann zeta function (i.e. the case of $\mathbb{Q}$ ) and the second the L-value at 2 of a CM elliptic curve defined over $\mathbb{Q}$ for regular primes. In the last case Bloch and Kato use an ad hoc method to describe the $p$-adic regulator of the K-theory. This does not extend to higher K-groups.

The regulator map to Deligne cohomology was computed by Deninger [Den2] with the help of the Eisenstein symbol. Here, the regulator can be described in terms of real analytic Eisenstein series (whence the name) and leads to a proof of the Beilinson conjecture for CM elliptic curves. For the Tamagawa number conjecture one needs an understanding of the $p$-adic regulator on the subspace of K-theory defined by the Eisenstein symbol. In an earlier paper Hu-Ki2] we established with A. Huber the relation of the $p$-adic regulator of the Eisenstein symbol with the specialization of the $p$-adic elliptic polylogarithm sheaf. The problem remains, to compute these specializations.

The elliptic polylogarithm is one of the most powerful tools in the study of special values of L-functions. All known cases of the Beilinson conjecture are proved or can be proved with specializations of the elliptic polylog. A universal property characterizes the polylog, which simplifies the explicit computations. So far, only the absolute Hodge realization of the elliptic polylog was understood, due to the extensive work of Beilinson and Levin Be-Le]. Missing was a theory of the $p$-adic realization, which give manageable étale cohomology classes. Such an explicit realization is known in the cyclotomic case. The approach there, mainly due to Deligne [Del2], uses torsors over $\mathbb{G}_{m} \backslash 1$ which 
are ramified in 1. This is not transferable to the elliptic case, because such torsors over elliptic curves do not exist (there are no Galois coverings ramified in exactly one point due to compactness). In our approach we allow instead ramification at torsion points, constructing in fact torsors over $E \backslash E\left[l^{n}\right]$. But this is not the only change of point of view compared to the cyclotomic case. The question is also, what is the group whose torsors we have to consider. The right choice is the group of torsion points of the torus with character group the augmentation ideal of the group ring of $E\left[l^{n}\right]$.

It turns out that the elliptic polylogarithm is an inverse limit of $p^{r}$-torsion points of a certain one-motive, which is essentially the generalized Jacobian defined by the divisor of all $p^{r}$-torsion points on the elliptic curve $E$. The cohomology classes of the elliptic polylogarithm sheaf can then be described by classes of sections of certain line bundles. These sections are elliptic units and going carefully through the construction one finds an analog of the elliptic Soulé elements of [So2].

Now enters Iwasawa theory: By an idea of Kato, going back in part to earlier work of Soulé [So2], the étale cohomology groups can be described in terms of Iwasawa modules and Rubin's "main conjecture" [Ru2 allows to give a bound on the kernel and the cokernel of Soulés map from elliptic units to the étale cohomology. On the way we also need some of the tools developed by Rubin to prove the main conjecture and the whole theory is the second decisive input into the proof of the Tamagawa number conjecture.

Let us finally give a rough sketch of the contents of this paper. More overviews can be found at the beginning of each section. In the first section we recall the statement of the Tamagawa number conjecture and formulate our main result 1.1.5. After this we recall Deninger's construction of the Ktheory elements leading to the Beilinson conjecture for CM elliptic curves. Here we also reduce to the computation of the specialization of the elliptic polylogarithm sheaf by using our earlier work Hu-Ki2.

The second section reviews the "main conjecture" and relates the Iwasawa modules to étale cohomology.

The next two sections are independent of the rest of the paper. The third section introduces the elliptic polylog and its specializations. The approach follows the important paper Be-Le] but puts the emphasis on different aspects, which are important for our geometric construction of the elliptic polylog.

The technical heart of the paper is section four. Here we formulate the polylog as an inverse limit of torsion points of one motives. The cohomology classes of what we call "geometric polylog" are then computed as the classes of sections of certain line bundles. 
The last section puts the various results together and gives the proof of the main theorem 1.1.5.

It is a pleasure to thank Annette Huber for her constant encouragement and for the many discussions about the contents of this paper and related results. Pierre Colmez read a first version of this paper and pointed out some inaccuracies and gave valuable hints for improvement. I like to thank him warmly. Thanks go also to Christopher Deninger for insisting strongly that my computation of the $l$-adic elliptic polylog should be turned immediately into a proof of the Tamagawa number conjecture. Also I like to thank P. Schneider for clarifying some point in Iwasawa theory.

\section{The Bloch-Kato conjecture for CM-elliptic curves}

This part of the paper contains our first main result, the Bloch-Kato conjecture for CM elliptic curves. For the precise formulation of our result we refer to 1.1.5. This part is organized as follows: First we review the Bloch-Kato conjecture in the case of interest to us. Here we also formulate the main theorem. Then we recall the construction due to Deninger of elements in the K-theory of CM elliptic curves. These elements satisfy the Beilinson conjecture for these curves as was shown by Deninger. This is the starting point of our investigations of the Bloch-Kato conjecture. In the formulation of Kato, the conjecture is concerned with $\mathbb{Z}_{p}$-lattices of $\mathbb{Q}_{p}$-vector spaces namely étale cohomology groups. Using an idea of Soulé (the elliptic Soulé elements) and the machinery of the Iwasawa "main conjecture" developed by Rubin, we can describe the étale cohomology groups, or better the complexes computing them, fairly well. The Iwasawa "main conjecture" will be reviewed in section 2.1 and the description of the étale cohomology groups is in section 2.2. Finally, the last section contains the comparison between the elliptic Soulé elements and Deninger's K-theory elements. Here we need the results of part 1 and in particular theorem 4.2.9.

\subsection{The Tamagawa number conjecture of Bloch-Kato and the main theorem}

This section recalls the Bloch-Kato conjecture Bl-Ka about special values of L-functions in the formulation of Kato [Ka1] and [Ka2]. We review this only for certain weights, which suffices for our purpose. Then we formulate our main result. 


\subsubsection{The Tamagawa number conjecture in the formulation of Kato}

Let $X / K$ be a smooth proper variety over a number field $K$ with ring of integers $\mathcal{O}_{K}$. Fix integers $m \geq 0$ and $r$ such that $m-2 r \leq-3$ and $r>$ $\inf (m, \operatorname{dim}(X))$. Let $p$ be a prime number not equal to 2 . Let $S$ be a set of finite primes of $K$ containing the primes lying over $p$ and the ones where $X$ has bad reduction. Let $\mathcal{O}_{S}$ be $\mathcal{O}_{K}\left[\frac{1}{S}\right]$ the ring, where the primes in $S$ are inverted. Define $\operatorname{Gal}(\bar{K} / K)$-modules

$$
\begin{aligned}
V_{p} & :=H^{m}\left(X \times_{K} \bar{K}, \mathbb{Q}_{p}(r)\right) \\
T_{p} & :=H^{m}\left(X \times_{K} \bar{K}, \mathbb{Z}_{p}(r)\right)
\end{aligned}
$$

Let $j: \operatorname{Spec} K \rightarrow \operatorname{Spec} \mathcal{O}_{S}$ and define the $p$-adic realizations to be

$$
H_{p}^{i}:=H^{i}\left(\mathcal{O}_{S}, j_{*} T_{p}\right) .
$$

We will omit the $j_{*}$, if no confusion is likely. Define

$$
H_{h, \mathbb{Z}}:=H_{\text {sing }}^{m}\left(X \times_{\mathbb{Q}} \mathbb{C},(2 \pi i)^{r-1} \mathbb{Z}\right)^{+}
$$

where the + denotes the fixed part under $\operatorname{Gal}(\mathbb{C} / \mathbb{R})$ of the singular cohomology of $X$. Here $\operatorname{Gal}(\mathbb{C} / \mathbb{R})$ acts on $\mathbb{C}$ and on $(2 \pi i)^{r-1} \mathbb{Z}$.

Finally we need the K-theory of $X$ : Let

$$
H_{\mathcal{M}}=\left(K_{2 r-m-1}(X) \otimes \mathbb{Q}\right)^{(r)}
$$

be the $r$-th Adams eigenspace of the $2 r-m-1$-th Quillen K-theory of $X$. There are regulator maps due to Beilinson and Soulé

$$
r_{\mathcal{D}}: H_{\mathcal{M}} \otimes_{\mathbb{Q}} \mathbb{R} \rightarrow H_{h, \mathbb{Z}} \otimes_{\mathbb{Z}} \mathbb{R}
$$

and

$$
r_{p}: H_{\mathcal{M}} \otimes_{\mathbb{Q}} \mathbb{Q}_{p} \rightarrow H_{p}^{1} \otimes_{\mathbb{Z}_{p}} \mathbb{Q}_{p}
$$

called the Deligne regulator (see [Be1]) and the $p$-adic regulator (see [So2]).

Remark: Note that because of our assumption $r>\inf (m, \operatorname{dim}(X))$, the Deligne cohomology coincides with $H_{h, \mathbb{Z}} \otimes_{\mathbb{Z}} \mathbb{R}$ (cf. [Sch2] sequence $(*)$ on page $9)$. The same condition (together with $m-2 r \leq-3$ ) also guarantees that $H_{\text {lim }}^{i}=H_{p}^{i} \otimes_{\mathbb{Z}_{p}} \mathbb{Q}_{p}$ (cf. Ka2] 2.2.6 (4)).

Let us define local Euler factors for $X$. Let for a prime $\mathfrak{p} \nmid p$ in $\mathcal{O}_{K}$

$$
P_{\mathfrak{p}}\left(V_{p}, s\right):=\operatorname{det}_{\mathbb{Q}_{p}}\left(1-\operatorname{Fr}_{\mathfrak{p}} \mathrm{Np}^{-s} \mid V_{p}^{I_{\mathfrak{p}}}\right)
$$


be the characteristic polynomial of the geometric Frobenius $\operatorname{Fr}_{\mathfrak{p}}$ at $\mathfrak{p}$ on the invariants of $V_{p}$ under the inertia group $I_{\mathfrak{p}}$ at $\mathfrak{p}$. For $\mathfrak{p} \mid p$ set

$$
P_{\mathfrak{p}}\left(V_{p}, s\right):=\operatorname{det}_{\mathbb{Q}_{p}}\left(1-\phi_{\mathfrak{p}}^{-1} \mathrm{~Np}^{-s} \mid D_{\text {cris }}\left(V_{p}\right)\right)
$$

where $\left.D_{\text {cris }}\left(V_{p}\right)\right):=\left(V_{p} \otimes_{\mathbb{Q}_{p}} B_{\text {cris }}\right)^{\operatorname{Gal}\left(\overline{\mathbb{Q}}_{p} / \mathbb{Q}_{p}\right)}$ and $\phi_{\mathfrak{p}}$ is the arithmetic Frobenius. Define the L-function of $X$ as

$$
L_{S}\left(V_{p}, s\right):=\prod_{\mathfrak{p} \notin S} P_{\mathfrak{p}}\left(V_{p}, s\right)^{-1} .
$$

Let $V_{p}^{*}$ be the dual Galois module of $V_{p}$. We now give Kato's formulation of the Tamagawa number conjecture. Here and in the rest of the paper the determinants are taken in the sense of Knudsen and Mumford Kn-Mu.

Conjecture 1.1.1. (cf. Kag]) Let $p \neq 2$ be a prime number, $r, m$ and $S$ be as above. Assume that

$$
P_{\mathfrak{p}}\left(V_{p}^{*}(1), 0\right) \neq 0
$$

for all $\mathfrak{p} \in S$ and that $L_{S}\left(V_{p}^{*}(1), s\right)$ has an analytic continuation to all of $\mathbb{C}$. Then:

a) The maps $r_{\mathcal{D}}$ and $r_{p}$ are isomorphisms and $H_{p}^{2}$ is finite.

b) $\operatorname{dim}_{\mathbb{Q}}\left(H_{h, \mathbb{Z}} \otimes \mathbb{Q}\right)=\operatorname{ord}_{s=0} L_{S}\left(V_{p}^{*}(1), s\right)$.

c) Let $\eta \in \operatorname{det}_{\mathbb{Z}}\left(H_{h, \mathbb{Z}}\right)$ be a $\mathbb{Z}$-basis and let $e:=\operatorname{dim}_{\mathbb{Q}}\left(H_{h, \mathbb{Z}} \otimes \mathbb{Q}\right)$. There is an element $\xi \in \operatorname{det}_{\mathbb{Q}}\left(H_{\mathcal{M}}\right)$ such that

$$
r_{\mathcal{D}}(\xi)=\left(\lim _{s \rightarrow 0} s^{-e} L_{S}\left(V_{p}^{*}(1), s\right)\right) \eta .
$$

This is the "Beilinson conjecture".

d) Consider $r_{p}(\xi) \in \operatorname{det}_{\mathbb{Q}_{p}}\left(H_{p}^{1} \otimes_{\mathbb{Z}_{p}} \mathbb{Q}_{p}\right)$, then $r_{p}(\xi)$ is a basis of the $\mathbb{Z}_{p}$-lattice

$$
\operatorname{det}_{\mathbb{Z}_{p}}\left(R \Gamma\left(\mathcal{O}_{S}, T_{p}\right)\right)^{-1} \subset \operatorname{det}_{\mathbb{Q}_{p}}\left(R \Gamma\left(\mathcal{O}_{S}, V_{p}\right)[-1]\right) \cong \operatorname{det}_{\mathbb{Q}_{p}}\left(H_{p}^{1} \otimes_{\mathbb{Z}_{p}} \mathbb{Q}_{p}\right),
$$

i.e.

$$
\left[\operatorname{det}_{\mathbb{Z}_{p}}\left(H_{p}^{1}\right): r_{p}(\xi) \mathbb{Z}_{p}\right]=\#\left(H_{p}^{2}\right) .
$$

Remark: a) The assumption in the conjecture is true for abelian varieties with CM.

b) The space $H_{p}^{0}$ is zero for weight reasons.

c) Part b) follows from the expected shape of the functional equation, (see e.g. Sch2 proposition page 9).

As our knowledge of K-theory is limited, let us also formulate a weak version of the above conjecture. 
Conjecture 1.1.2. (weak form of conjecture 1.1.1) There is a subspace $H_{\mathcal{M}}^{\text {constr }}$ in $H_{\mathcal{M}}$ (the constructible elements of $H_{\mathcal{M}}$ ) such that

$\left.a^{\prime}\right) r_{\mathcal{D}}$ and $r_{p}$ restricted to $H_{\mathcal{M}}^{\text {constr }}$ are isomorphisms and $H_{p}^{2}$ is finite.

b') same as b)

$\left.c^{\prime}\right)$ There is an element $\xi \in \operatorname{det}_{\mathbb{Q}}\left(H_{\mathcal{M}}^{\text {constr }}\right)$ such that

$$
r_{\mathcal{D}}(\xi)=\left(\lim _{s \rightarrow 0} s^{-e} L_{S}\left(V_{p}^{*}(1), s\right)\right) \eta .
$$

d') The element $r_{p}(\xi)$ is a basis of the $\mathbb{Z}_{p}$-lattice

$$
\operatorname{det}_{\mathbb{Z}_{p}}\left(R \Gamma\left(\mathcal{O}_{S}, T_{p}\right)\right)^{-1} \subset \operatorname{det}_{\mathbb{Q}_{p}}\left(R \Gamma\left(\mathcal{O}_{S}, V_{p}\right)[-1]\right) \cong \operatorname{det}_{\mathbb{Q}_{p}}\left(H_{p}^{1} \otimes_{\mathbb{Z}_{p}} \mathbb{Q}_{p}\right) .
$$

\subsubsection{Elliptic curves with CM}

Before we formulate the main theorem, we introduce the elliptic curves we want to consider. We follow the notations and conventions in Deninger [Den1]. Let $K$ be an imaginary quadratic field with ring of integers $\mathcal{O}_{K}$. Let $E / K$ be an elliptic curve with $\mathrm{CM}$ by $\mathcal{O}_{K}$. Note that this implies that the class number of $K$ is one. We fix an isomorphism

$$
\vartheta: \mathcal{O}_{K} \cong \operatorname{End}_{K}\left(E_{K}\right)
$$

such that for $\omega \in \Gamma\left(E_{K}, \Omega_{E_{K} / K}\right)$ and $\alpha \in \mathcal{O}_{K}$ we have $\vartheta^{*}(\alpha) \omega=\alpha \omega$. We fix also an embedding of $K$ into $\mathbb{C}$, such that the algebraic $j$-invariant of $E$ is the same as the corresponding complex analytic $j$-invariant of $\mathcal{O}_{K}$. Let us denote by

$$
\psi: \mathbb{A}_{K}^{*} \rightarrow K^{*} \subset \mathbb{C}^{*}
$$

the CM-character or Serre-Tate character of $E_{K}$ and let $\mathfrak{f}$ be its conductor. The elliptic curve $E$ has bad reduction precisely at the primes dividing $\mathfrak{f}$. Denote by $\bar{\psi}$ the complex conjugate character. Its conductor is also $\mathfrak{f}$.

Definition 1.1.3. Fix a prime number $p$. We let $S$ be the set of primes in $K$ dividing $p \mathfrak{f}$.

Associated to $\psi$ is an L-series

$$
L_{S}(\psi, s)=\prod_{\mathfrak{p} \nmid p \mathfrak{f}} \frac{1}{1-\frac{\psi(\mathfrak{p})}{N \mathfrak{p}^{s}}} .
$$

We want to relate this to the L-function $L_{S}(E, s)$ of $E$. Recall the fundamental result of Deuring: 
Theorem 1.1.4. (see $S$ TII 10.5.) Let $L_{S}(E / K, s):=L_{S}\left(V_{p}, s\right)$ be the $L$ series of the Galois representation $V_{p}:=H^{1}\left(E \times_{K} \bar{K}, \mathbb{Q}_{p}\right)$ as defined in section 1.1.1. Then

$$
L_{S}(E / K, s)=L_{S}(\psi, s) L_{S}(\bar{\psi}, s) .
$$

Let $T_{p} E=\lim _{n} E\left[p^{n}\right]$ be the Tate-module of $E$. This is a $\operatorname{Gal}(\bar{K} / K)$ module. Then $H^{1}\left(E \times_{K} \bar{K}, \mathbb{Z}_{p}\right) \cong \operatorname{Hom}\left(T_{p} E, \mathbb{Z}_{p}\right) \cong T_{p} E(-1)$, where $\mathcal{O}_{p}:=$ $\mathcal{O}_{K} \otimes \mathbb{Z}_{p}$ acts now conjugate linear on $T_{p} E$. There is a canonical isomorphism

$$
H^{1}\left(E \times_{\mathbb{Q}} \mathbb{C},(2 \pi i)^{r} \mathbb{Z}\right)^{+} \cong H^{1}\left(E \times_{K} \mathbb{C},(2 \pi i)^{r} \mathbb{Z}\right),
$$

where we used the fixed embedding $K \subset \mathbb{C}$. Let

$$
H_{\mathcal{M}}^{i}(E, j):=\left(K(E)_{2 j-i} \otimes \mathbb{Q}\right)^{(j)}
$$

be the $2 j-i$-th Quillen K-theory of $E$.

For $m=1, r=k+2$ with $k \geq 0$ in the notation of 1.1.1 we have

$$
\begin{aligned}
& H_{p}^{i}=H^{i}\left(\mathcal{O}_{S}, T_{p} E(k+1)\right) \\
& H_{h, \mathbb{Z}}=H^{1}\left(E \times_{K} \mathbb{C},(2 \pi i)^{k+1} \mathbb{Z}\right) \\
& H_{\mathcal{M}}=H_{\mathcal{M}}^{2}(E, k+2) .
\end{aligned}
$$

Note that on all these spaces we have a canonical $\mathcal{O}_{K}$-action and that $H_{h, \mathbb{Z}}$ is an $\mathcal{O}_{K}$-module of rank 1 . It is a result of Jannsen ([Ja2] corollary 1) that if $H_{p}^{2}$ is finite then the free part of $H_{p}^{1}$ is an $\mathcal{O}_{K}$-module of rank 1.

\subsubsection{The main theorem}

Now we can formulate our main result. We let $\mathcal{O}_{p}:=\mathcal{O}_{K} \otimes \mathbb{Z}_{p}$.

Theorem 1.1.5. Let $p \neq 2,3$ and $p \nmid \mathrm{N}_{K / Q} \mathfrak{f}$ and $k \geq 0$. Then, there is an $\mathcal{O}_{K}$ submodule $\mathcal{R}_{\psi} \subset H_{\mathcal{M}}$ of rank 1 such that

a) $\operatorname{det}_{\mathcal{O}_{K}}\left(r_{\mathcal{D}}\left(\mathcal{R}_{\psi}\right)\right) \cong L_{S}^{*}(\bar{\psi},-k) \operatorname{det}_{\mathcal{O}_{K}}\left(H_{h, \mathbb{Z}}\right)$ in $\operatorname{det}_{\mathcal{O}_{K} \otimes \mathbb{R}}\left(H_{h, \mathbb{Z}} \otimes \mathbb{R}\right)$ and

b) The map $r_{p}$ induces an isomorphism

$$
\operatorname{det}_{\mathcal{O}_{p}}\left(\mathcal{R}_{\psi}\right) \cong \operatorname{det}_{\mathcal{O}_{p}}\left(R \Gamma\left(\mathcal{O}_{S}, T_{p} E(k+1)\right)\right)^{-1}
$$

Here $L^{*}(\bar{\psi},-k)=\lim _{s \rightarrow-k} \frac{L(\bar{\psi}, s)}{s}$ denotes the leading coefficient of the Taylor series of $L(\bar{\psi}, s)$ at $-k$. Moreover, if $H_{p}^{2}$ is finite, $r_{p}$ is injective on $\mathcal{R}_{\psi}$ and

$$
\operatorname{det}_{\mathcal{O}_{p}} H_{p}^{1} / r_{p}\left(\mathcal{R}_{\psi}\right) \cong \operatorname{det}_{\mathcal{O}_{p}} H_{p}^{2} \text {. }
$$


Remark: i) Part a) was proven by Deninger in [Den1. This is the Beilinson conjecture for Hecke characters.

ii) For $k=0$ and CM elliptic curves defined over $\mathbb{Q}$ with CM by $\mathcal{O}_{K}$ and $p$ regular, part b) was proven in Bl-Ka. They used an ad hoc computation of the $p$-adic realization of the K-theory elements, which does not generalize.

iii) Let us explain part b) in more detail: The Soulé regulator

$$
r_{p}: \mathcal{R}_{\psi} \rightarrow H_{p}^{1} \otimes \mathbb{Q}_{p}
$$

extends to a map to $R \Gamma\left(\mathcal{O}_{S}, T_{p} E(k+1)\right)[-1] \otimes \mathbb{Q}_{p}$ because $H_{p}^{0}$ is zero for weight reasons. The determinant of this complex has as $\mathcal{O}_{p}$-lattice the determinant of $R \Gamma\left(\mathcal{O}_{S}, T_{p} E(k+1)\right)[-1]$, which is $\operatorname{det}_{\mathcal{O}_{p}}\left(R \Gamma\left(\mathcal{O}_{S}, T_{p} E(k+1)\right)\right)^{-1}$. Part b) means that the determinant of the complex

$$
\mathcal{R}_{\psi} \stackrel{r_{p}}{\longrightarrow} R \Gamma\left(\mathcal{O}_{S}, T_{p} E(k+1)\right)[-1]
$$

is trivial. If $H_{p}^{2}$ is finite, and $r_{p} \otimes \mathbb{Q}_{p}$ an isomorphism, we get from this $\operatorname{det}_{\mathcal{O}_{p}}\left(H_{p}^{1} / r_{p}\left(\mathcal{R}_{\psi}\right)\right) \cong \operatorname{det}_{\mathcal{O}_{p}} H_{p}^{2}$.

iv) Note that $H_{p}^{2}$ is finite for almost all $k \geq 0$ or for $p$ regular. See the next section for remarks on the finiteness of $H_{p}^{2}$.

v) From this result for the set of primes $S$ we get it for all other set of primes which contain $S$. This follows from Ka2 4.11.

As a corollary we get a result about $\mathbb{Z}_{p}$ determinants and the L-function of $E / K$ :

Corollary 1.1.6. Under the conditions of the theorem

a) $\operatorname{det}_{\mathbb{Z}}\left(r_{\mathcal{D}}\left(\mathcal{R}_{\psi}\right)\right)=L_{S}^{*}(E / K,-k) \operatorname{det}_{\mathbb{Z}}\left(H_{h, \mathbb{Z}}\right)$

and that

b) $\operatorname{det}_{\mathbb{Z}_{p}}\left(r_{p}\left(\mathcal{R}_{\psi}\right)\right)=\operatorname{det}_{\mathbb{Z}_{p}}\left(R \Gamma\left(\mathcal{O}_{S}, T_{p} E(k+1)\right)\right)^{-1}$.

Here $L^{*}(E / K,-k)=\lim _{s \rightarrow-k} \frac{L(E / K, s)}{s^{2}}$ denotes the leading coefficient of the Taylor series of $L(E / K, s)$ at $-k$.

Proof. This follows from the theorem and the remark that if we multiply an $\mathcal{O}_{K}$-module with an element $L^{*}(\bar{\psi},-k)$ from $\mathcal{O}_{K} \otimes \mathbb{R}$, then the determinant is multiplied by the norm $\mathrm{N}_{\mathcal{O}_{K} \otimes \mathbb{R} / \mathbb{R}}\left(L^{*}(\bar{\psi},-k)\right)$. But $\mathrm{N}_{\mathcal{O}_{K} \otimes \mathbb{R} / \mathbb{R}}\left(L^{*}(\bar{\psi},-k)\right)=$ $L^{*}(E / K,-k)$. Part b) is obvious.

Here is a short overview of the proof: We start by recalling Deninger's definition of K-theory elements and his main result about the relation of these to the $L$-value. Then we use an idea of Soulé to construct a submodule of

$$
H^{1}\left(\mathcal{O}_{S}, T_{p} E(k+1)\right)
$$


via elliptic units. Iwasawa theory allows us to compute the index of this submodule. The proof concludes with the comparison of this submodule and $\mathcal{R}_{\psi}$. This is the main step in the proof which needs the theory developed in part 1 and in particular the explicit description of the elliptic polylogarithm of theorem 4.2.9. The injectivity of $r_{p}$ in the case that $H_{p}^{2}$ is finite, will be proved in section 5.2.2.

\subsubsection{Some remarks concerning the finiteness of $H^{2}$}

Note that we do not prove that $H_{p}^{2}$ is finite. Nevertheless, there is the following result of Soulé:

Theorem 1.1.7. (So1 1.5 proposition 3) For fixed $p$ the group

$$
H^{2}\left(\mathcal{O}_{S}, T_{p} E(k+1)\right)
$$

is finite for almost all $k$.

For regular $p$, we have results of Soulé and Wingberg:

Theorem 1.1.8. (Sog 3.3.2, Win cor. 2) Let $p$ be a regular prime for $E$ (see e.g.SSOZ 3.3.1 for the definition of regular), then

$$
H^{2}\left(\mathcal{O}_{S}, E\left[p^{\infty}\right](k+1)\right)=0 .
$$

It is easy to see that this vanishing implies the finiteness of $H_{p}^{2}$ (cf. Ja2 lemma 1).

Remark: In Ja2 it is conjectured that $H_{p}^{2}$ is always finite.

\subsection{Review of the Deninger elements for CM elliptic curves over an imaginary quadratic field}

Here we describe briefly the construction by Deninger Den1] of the elements in K-theory, which interpret the L-value up to rational numbers as predicted by Beilinson's conjecture.

We are interested in the L-values $L(\bar{\psi}, k+2)$ with $k \geq 0$. Thus according to 1.1.1 we need an element in $H_{\mathcal{M}}^{2}(E, k+2)$.

\subsubsection{The Deninger-Beilinson construction}

We fix an algebraic differential $\omega \in H^{0}\left(E, \Omega_{E / K}\right)$ and let $\Gamma$ be its period lattice. Then we have an isomorphism

$$
\begin{aligned}
E(\mathbb{C}) & \rightarrow \mathbb{C} / \Gamma \\
z & \mapsto \int_{0}^{z} \omega
\end{aligned}
$$


using the fixed embedding $K \subset \mathbb{C}$. This isomorphism is equivariant for the

action of complex multiplication and because $j(E)=j\left(\mathcal{O}_{K}\right)$ the lattice is of the form $\Gamma=\Omega \mathcal{O}_{K}$ for some $\Omega \in \mathbb{C}^{*}$. Fix an $\mathcal{O}_{K}$ generator $\gamma \in H_{1}(E(\mathbb{C}), \mathbb{Z})$, then

$$
\Omega=\int_{\gamma} \omega
$$

Recall that $\mathfrak{f}$ is the conductor of $\psi$ and the locus of bad reduction of $E$. Let $\mathbb{Z}[E[\mathfrak{f}] \backslash 0]$ be the group of divisors with support in the $\mathfrak{f}$-torsion points without 0 of $E$, defined over $K$. Beilinson defines a map:

Theorem 1.2.1. (Bea) There is a non-zero map, a variant of the Eisenstein symbol,

$$
\mathbb{Z}[E[\mathfrak{f}] \backslash 0] \stackrel{\mathcal{E}_{\mathcal{M}}^{2 k+1}}{\longrightarrow} H_{\mathcal{M}}^{2 k+2}\left(E^{2 k+1}, 2 k+2\right),
$$

where $E^{n}:=E \times_{K} \ldots \times_{K} E$.

Deninger constructs a projector

$$
\mathcal{K}_{\mathcal{M}}: H_{\mathcal{M}}^{2 k+2}\left(E^{2 k+1}, 2 k+2\right) \rightarrow H_{\mathcal{M}}^{2}(E, k+2)
$$

as follows: Let $d_{K}$ be the discriminant of $K$ and $\sqrt{d_{K}}$ be a square root of $d_{K}$. Complex multiplication gives a map

$$
\delta=\left(\mathrm{id}, \vartheta\left(\sqrt{d_{K}}\right)\right): E \rightarrow E \times_{K} E
$$

and taking this $k$-times gives $\delta^{k} \times$ id $: E^{k} \times_{K} E \rightarrow E^{2 k} \times_{K} E$. Then

$$
\mathcal{K}_{\mathcal{M}}=\operatorname{pr}_{*} \circ\left(\delta^{k} \times \mathrm{id}\right)^{*}
$$

where pr is the projection $E^{k} \times{ }_{K} E \rightarrow E$ onto the last component. Hence we get a map

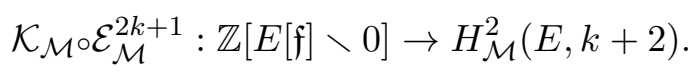

\subsubsection{The Beilinson conjecture for CM elliptic curves}

Following Deninger we define an element $\beta$ in $\mathbb{Z}[E[\mathfrak{f}] \backslash 0]$. Let $K(\mathfrak{f})$ be the ray class field associated to $\mathfrak{f}$ and note that $K(\mathfrak{f})=K(E[\mathfrak{f}])$.

Let $f$ be a generator of $\mathfrak{f}$. Then

$$
\Omega f^{-1} \in \mathfrak{f}^{-1} \Gamma
$$


defines an element in $E[\mathfrak{f}](K(\mathfrak{f}))$. This gives a divisor $\left(\Omega f^{-1}\right)$ in $\mathbb{Z}[E[\mathfrak{f}] \backslash 0]$ defined over $K(\mathfrak{f})$ on which the Galois group $\operatorname{Gal}(K(\mathfrak{f}) / K)$ acts. We define:

$$
\beta:=\mathrm{N}_{K(\mathfrak{f}) / K}\left(\left(\Omega f^{-1}\right)\right) .
$$

This is a divisor defined over $K$. Recall that $\gamma$ is an $\mathcal{O}_{K}$ generator of $H_{1}(E(\mathbb{C}), \mathbb{Z})$. By Poincaré duality we have an isomorphism (conjugate linear for the $\mathcal{O}_{K^{-}}$ action)

$$
H^{1}(E(\mathbb{C}), \mathbb{Z}(k+1)) \cong \operatorname{Hom}\left(H^{1}(E(\mathbb{C}), \mathbb{Z}), \mathbb{Z}(k)\right)=H_{1}(E(\mathbb{C}), \mathbb{Z}(k)) .
$$

Denote by $\eta$ the $\mathcal{O}_{K}$ generator of $H^{1}(E(\mathbb{C}), \mathbb{Z}(k+1))$ corresponding to $(2 \pi i)^{k} \gamma$ under this isomorphism. We can now formulate the main result of [Den1] in our case:

Theorem 1.2.2. (Den1 thm. 11.3.2) Let $\beta$ and $\eta$ be as above and define

$$
\xi:=(-1)^{k-1} \frac{(2 k+1) !}{2^{k-1}} \frac{L_{p}(\bar{\psi},-k)^{-1}}{\psi(f) \mathrm{N}_{K / \mathbb{Q}} \mathfrak{f}^{k}} \mathcal{K}_{\mathcal{M}} \mathcal{E}_{\mathcal{M}}^{2 k+1}(\beta) \in H_{\mathcal{M}}^{2}(E, k+2),
$$

where $L_{p}(\bar{\psi},-k)$ is the Euler factor of $\bar{\psi}$ at $p$, evaluated at $-k$. Then

$$
r_{\mathcal{D}}(\xi)=L_{S}^{*}(\bar{\psi},-k) \eta \in H^{1}\left(E \times_{K} \mathbb{C},(2 \pi i)^{k+1} \mathbb{R}\right),
$$

where $L_{S}^{*}(\bar{\psi},-k)=\lim _{s \rightarrow-k} \frac{L_{S}(\bar{\psi}, s)}{s}$.

Note that $L_{\mathfrak{p}}(\bar{\psi},-k)=1$, if $\mathfrak{p} \mid \mathfrak{f}$. We can now define the space $\mathcal{R}_{\psi}$ of the main theorem 1.1 .5

Definition 1.2.3. We define

$$
\mathcal{R}_{\psi}:=\xi \mathcal{O}_{K} \subset H_{\mathcal{M}}^{2}(E, k+2)
$$

to be the $\mathcal{O}_{K}$-submodule of $H_{\mathcal{M}}^{2}(E, k+2)$ generated by $\xi$.

Note that by the above theorem, $\mathcal{R}_{\psi}$ is an $\mathcal{O}_{K}$-modules of rank 1.

Corollary 1.2.4. With the above notation

$$
r_{\mathcal{D}}\left(\operatorname{det}_{\mathbb{Z}}\left(\mathcal{R}_{\psi}\right)\right)=L_{S}^{*}(E / K,-k) \operatorname{det}_{\mathbb{Z}}\left(H^{1}(E(\mathbb{C}), \mathbb{Z}(k+1))\right) .
$$

where $S$ is the set of primes in $K$ dividing $p \mathfrak{f}$.

Proof. This follows from the theorem and the remark that if we multiply an $\mathcal{O}_{K}$-module with an element $L_{S}^{*}(\bar{\psi},-k)$ from $\mathcal{O}_{K} \otimes \mathbb{R}$, then the determinant is multiplied by the norm $\mathrm{N}_{\mathcal{O}_{K} \otimes \mathbb{R} / \mathbb{R}}\left(L_{S}^{*}(\bar{\psi},-k)\right)$. But $\mathrm{N}_{\mathcal{O}_{K} \otimes \mathbb{R} / \mathbb{R}}\left(L_{S}^{*}(\bar{\psi},-k)\right)=$ $L_{S}^{*}(E / K,-k)$. 


\subsubsection{The space $r_{p}\left(\mathcal{R}_{\psi}\right)$ in terms of the specialization of the elliptic polylog}

Recall from definition 1.2 .3 that the space $\mathcal{R}_{\psi}$ is generated as an $\mathcal{O}_{K}$-module by the element $\xi$ from theorem 1.2.2. The element $\xi$ is up to some factors of

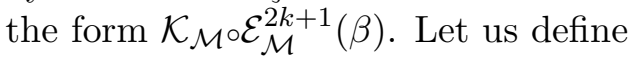

$$
t:=\Omega f^{-1}
$$

with the notation from 1. This is an $\mathrm{N}_{K / \mathbb{Q}} \mathfrak{f}$-division point. Then we have $\beta=\mathrm{N}_{K(\mathfrak{f}) / K}((t))$. Now let

$$
\left(\beta^{*} \mathcal{P}_{\mathrm{Ol}_{\mathbb{Q}_{p}}}\right)^{2 k+1} \in H^{1}\left(\mathcal{O}_{S}, \operatorname{Sym}^{2 k+1} \mathcal{H}_{\mathbb{Q}_{p}}(1)\right)
$$

be the specialization of the polylogarithm as defined in 3.5.9. We have the following comparison theorem:

Theorem 1.2.5. There is an equality

$$
r_{p}\left(\mathcal{E}_{\mathcal{M}}^{2 k+1}(\beta)\right)=-\mathrm{N} \mathfrak{f}^{4 k+2}\left(\beta^{*} \mathcal{P}_{\mathrm{ol}_{\mathbb{Q}_{p}}}\right)^{2 k+1}
$$

in $H^{1}\left(\mathcal{O}_{S}, \operatorname{Sym}^{2 k+1} \mathcal{H}_{\mathbb{Q}_{p}}(1)\right)$.

Proof. The formula is the combination of two results: Theorem 2.2.4 in Hu-Ki2, which states that

$$
r_{p}\left(\mathcal{E}_{i s_{\mathcal{M}}^{2 k+1}}(\varrho \beta)\right)=-\mathrm{N} \mathfrak{f}^{2 k}\left(\beta^{*} \mathcal{P}_{\mathrm{ol}} \mathbb{Q}_{p}\right)^{2 k+1}
$$

where $\mathcal{E} i s_{\mathcal{M}}^{2 k+1}$ is Beilinson's Eisenstein symbol and $\varrho$ the horospherical map. Note that what is here called $\left(\beta^{*} \mathcal{P}_{\mathrm{ol}_{\mathbb{Q}_{p}}}\right)^{2 k+1}$ is in loc. cit. $\left(\beta^{*} \mathcal{P}_{\mathrm{ol}_{\mathbb{Q}_{p}}}\right)^{2 k+2}$. Furthermore, according to [Den2] formula 3.35.,

$$
\mathcal{E}_{\mathcal{M}}^{2 k+1}(\beta)=\mathrm{Nf}^{2 k+2} \mathcal{E} i s_{\mathcal{M}}^{2 k+1}(\varrho \beta)
$$

Note that the formula 3.35. in Den2] uses an other normalization of the horospherical map and that there is a factor $\mathrm{Nf}$ missing because of a wrong normalization of the residue map (the residue of $\frac{d q}{q}$ in formula (3.7) in loc. cit. is not 1 but $N$ ).

We have now two tasks: To compute the specialization of the elliptic poly$\log$ and to identify the étale cohomology groups to compute the "index" of $r_{p}\left(\mathcal{R}_{\psi}\right)$ in $H^{1}\left(\mathcal{O}_{S}, \operatorname{Sym}^{2 k+1} \mathcal{H}_{\mathbb{Z}_{p}}(1)\right)$. The answer to these problems involves elliptic units and we will start to use Iwasawa theory and Rubin's proof of the main conjecture to identify the the étale cohomology groups. 


\section{Iwasawa theory}

This section treats the relation between certain Iwasawa modules and étale cohomology. Rubin's "main conjecture" is used in an essential way. We first review the results of Rubin and then use an idea of Soulé to produce elements in étale cohomology using elliptic units. An idea of Kato, which partly goes back to Soulé as well, allows to compare the elliptic units and étale cohomology. The main result of this part is theorem 2.2.11.

\subsection{Review of the "main conjecture" of Iwasawa theory for CM elliptic curves}

In this section we review the "main conjecture" of Iwasawa theory for CM elliptic curves proved by Rubin Ru2. This will be used in section 2.2 to reduce the Tamagawa number conjecture to an "index computation".

\subsubsection{Definition of the Iwasawa modules}

We follow Rubin Ru2]: Let $E / K$ be as before an elliptic curve with $\mathrm{CM}$ by $\mathcal{O}_{K}, K$ an imaginary quadratic field. We fix an embedding of $K$ into $\mathbb{C}$ and view $K$ as a subfield of $\mathbb{C}$. Fix a prime $p \nmid \# \mathcal{O}_{K}^{*}$ and a prime $\mathfrak{p}$ of $\mathcal{O}_{k}$ lying over $p$ and denote by $E\left[p^{n}\right]$ the $p^{n}$-torsion points of $E$. Let $K_{n}:=K\left(E\left[p^{n+1}\right]\right)$ be the extension field defined by these torsion points and $K_{\infty}:=\lim _{n} K_{n}$. Denote the ring of integers in these fields by $\mathcal{O}_{n}$ (resp. $\left.\mathcal{O}_{\infty}\right)$. Then $\Delta:=$ $\operatorname{Gal}\left(K_{0} / K\right)$ has order prime to $p$ and $\Gamma:=\operatorname{Gal}\left(K_{\infty} / K_{0}\right)$ is isomorphic to $\mathbb{Z}_{p}^{2}$. Let $\mathcal{G}:=\operatorname{Gal}\left(K_{\infty} / K\right)$ be the Galois group of the extension $K_{\infty} / K$. Then $\mathcal{G} \cong \Delta \times \Gamma$. Define $\mathcal{A}_{n}$ to be the $p$-part of the ideal class group of $K_{n}, \mathcal{E}_{n}$ to be the group of global units $\mathcal{O}_{n}^{*}$ of $K_{n}$ and $\mathcal{U}_{n}^{\mathfrak{p}}$ the local units of $K_{n} \otimes_{K} K_{\mathfrak{p}}$ which are congruent to 1 modulo the primes above $\mathfrak{p}$. For every prime $v$ of $K_{n}$ above $\mathfrak{p}$ there is an exact sequence

$$
1 \rightarrow \mathcal{U}_{n, v} \rightarrow K_{n, v}^{*} \rightarrow \mathbb{Z} \times \kappa_{n}^{*} \rightarrow 1
$$

and $\mathcal{U}_{n}^{\mathfrak{p}}=\bigoplus_{v \mid \mathfrak{p}} \mathcal{U}_{n, v}$. Here $\mathcal{U}_{n, v}$ are the local units congruent to 1 modulo $v$ and $\kappa_{n}$ is the residue class field of $K_{n, v}$. Let $\mathcal{C}_{n}$ be the elliptic units in $K_{n}$ as defined in Ru2 paragraph 1 . We recall their definition. For every ideal $\mathfrak{a} \in \mathcal{O}_{K}$ prime to 6 consider the function $\theta_{\mathfrak{a}}(z)$ that will be defined in 4.2.2. The function $\theta_{\mathfrak{a}}(z)$ is a 12-th root of the function in deSh II.2.4.

Let $t:=\Omega f^{-1}$ and $\mathfrak{a}$ be an ideal prime to $6 \mathfrak{f}$. 
Definition 2.1.1. (cf.Ru3 11.2) Let $C_{n}$ be the subgroup of units generated over $\mathbb{Z}\left[\operatorname{Gal}\left(K_{n} / K\right)\right]$ by

$$
\prod_{\sigma \in \operatorname{Gal}(K(\mathfrak{f}) / K)} \theta_{\mathfrak{a}}\left(t^{\sigma}+h_{n}\right),
$$

where $\mathfrak{a}$ runs through all ideals prime to $6 p \mathfrak{f}, K(\mathfrak{f})$ is the ray class field defined by $\mathfrak{f}$ and $h_{n}$ is a primitive $p^{n}$-torsion point. Define

$$
\mathcal{C}_{n}:=\mu_{\infty}\left(K_{n}\right) C_{n}
$$

the group of elliptic units of $K_{n}$.

Denote by $\overline{\mathcal{E}}_{n}$ and $\overline{\mathcal{C}}_{n}$ the closures of $\mathcal{E}_{n} \cap \mathcal{U}_{n}^{\mathfrak{p}}$ resp. $\mathcal{C}_{n} \cap \mathcal{U}_{n}^{\mathfrak{p}}$ in $\mathcal{U}_{n}^{\mathfrak{p}}$. Finally define

$$
\mathcal{A}_{\infty}:=\lim _{n} \mathcal{A}_{n}, \quad \overline{\mathcal{E}}_{\infty}:=\lim _{n} \overline{\mathcal{E}}_{n}, \quad \overline{\mathcal{C}}_{\infty}:=\lim _{n} \overline{\mathcal{C}}_{n}, \quad \mathcal{U}_{\infty}^{\mathfrak{p}}:=\lim _{n} \mathcal{U}_{n}^{\mathfrak{p}}
$$

where the limits are taken with respect to the norm maps. Denote by $M_{\infty}^{\mathfrak{p}}$ the maximal abelian $p$-extension of $K_{\infty}$ which is unramified outside of the primes above $\mathfrak{p}$, and write $\mathcal{X}_{\infty}^{\mathfrak{p}}:=\operatorname{Gal}\left(M_{\infty}^{\mathfrak{p}} / K_{\infty}\right)$. Global class field theory gives an exact sequence

$$
0 \rightarrow \overline{\mathcal{E}}_{\infty} / \overline{\mathcal{C}}_{\infty} \rightarrow \mathcal{U}_{\infty}^{\mathfrak{p}} / \overline{\mathcal{C}}_{\infty} \rightarrow \mathcal{X}_{\infty}^{\mathfrak{p}} \rightarrow \mathcal{A}_{\infty} \rightarrow 0
$$

Define the Iwasawa algebra

$$
\mathbb{Z}_{p}[[\mathcal{G}]]:=\lim _{n} \mathbb{Z}_{p}\left[\left[\operatorname{Gal}\left(K_{n} / K\right)\right]\right]
$$

this has an action of $\mathbb{Z}_{p}[\Delta]$. For any irreducible $\mathbb{Z}_{p}$-representation $\chi$ of $\Delta$, let

$$
e_{\chi}:=\frac{1}{\# \Delta} \sum_{\tau \in \Delta} \operatorname{Tr}(\chi(\tau)) \tau^{-1} \in \mathbb{Z}_{p}[\Delta]
$$

and let for every $\mathbb{Z}_{p}[\Delta]$-module $Y$ be $Y^{\chi}:=e_{\chi} Y$ be the $\chi$-isotypical component. In particular we define

$$
\Lambda^{\chi}:=\mathbb{Z}_{p}[[\mathcal{G}]]^{\chi}=R_{\chi}[[\Gamma]]
$$

where $R_{\chi}$ is the ring of integers in the unramified extension of $\mathbb{Z}_{p}$ of degree $\operatorname{dim}(\chi)$. As we will work with $\mathbb{Z}_{p}[[\Gamma]] \otimes \mathcal{O}_{p}$-modules, we let

$$
\Lambda:=\mathcal{O}_{p}[[\Gamma]] \text {. }
$$

Then $\overline{\mathcal{E}}_{\infty}^{\chi}, \overline{\mathcal{C}}_{\infty}^{\chi}, \mathcal{U}_{\infty}^{\mathfrak{p} \chi}, \mathcal{A}_{\infty}^{\chi}$ and $\mathcal{X}_{\infty}^{\chi}$ are finitely generated $\Lambda^{\chi}$-modules (see Ru2 paragraph 5). The modules $\mathcal{A}_{\infty}^{\chi}$ and $\overline{\mathcal{E}}_{\infty}^{\chi} / \overline{\mathcal{C}}_{\infty}^{\chi}$ are even torsion $\Lambda^{\chi}$-modules. 


\subsubsection{Rubin's "main conjecture" for imaginary quadratic fields}

We have the following lemma due to Kato:

Lemma 2.1.2. (see Kag proposition 6.1.) Let $Y$ be a finitely generated torsion $\Lambda^{\chi}$-module. Then

$$
\operatorname{det}_{\Lambda \chi}(Y)=\operatorname{char}(Y)
$$

where $\operatorname{char}(Y)$ is the usual characteristic ideal in Iwasawa theory (see e.g. Rug] paragraph 4) and the determinant is taken in the sense of [Kn-Mu].

With this lemma we can formulate the main result of [Ru2 as follows:

Theorem 2.1.3. (Rug theorem 4.1.) Let $p \nmid \# \mathcal{O}_{K}^{*}$.

i) Suppose that $p$ splits in $K$, then

$$
\operatorname{det}_{\Lambda \chi}\left(\mathcal{A}_{\infty}^{\chi}\right)=\operatorname{det}_{\Lambda \chi}\left(\overline{\mathcal{E}}_{\infty}^{\chi} / \overline{\mathcal{C}}_{\infty}^{\chi}\right) .
$$

ii) Suppose that $p$ remains prime or ramifies in $K$ and that $\chi$ is nontrivial on the decomposition group of $\mathfrak{p}$ in $\Delta$, then

$$
\operatorname{det}_{\Lambda \chi}\left(\mathcal{A}_{\infty}^{\chi}\right)=\operatorname{det}_{\Lambda \chi}\left(\overline{\mathcal{E}}_{\infty}^{\chi} / \overline{\mathcal{C}}_{\infty}^{\chi}\right) .
$$

Using the theory of the determinant and the exact sequence (3) we get:

Corollary 2.1.4. In the situation of the theorem 2.1.3,

$$
\operatorname{det}_{\Lambda \chi}\left(\mathcal{X}_{\infty}^{\mathfrak{p} \chi}\right)=\operatorname{det}_{\Lambda \chi}\left(\mathcal{U}_{\infty}^{\mathfrak{p} \chi} / \overline{\mathcal{C}}_{\infty}^{\chi}\right) .
$$

We need a variant of this. Let $\mathcal{X}_{\infty}$ be the Galois group of the maximal abelian $p$-extension $M_{\infty}^{p}$ of $K_{\infty}$ which is unramified outside of the primes above $p$. In the case where $p$ is inert or ramified this is the same as $\mathcal{X}_{\infty}^{\mathfrak{p}}$. Define also

$$
\mathcal{U}_{\infty}:=\mathcal{U}_{\infty}^{\mathfrak{p}} \times \mathcal{U}_{\infty}^{\mathfrak{p}^{*}}
$$

if $p=\mathfrak{p p}^{*}$ is split, and

$$
\mathcal{U}_{\infty}:=\mathcal{U}_{\infty}^{\mathfrak{p}}
$$

if $p$ is inert or ramified. Let similarly $\mathcal{Y}_{n}$ be the $p$-adic completion of $\left(K_{n} \otimes \mathbb{Q}_{p}\right)^{*}$ and $\mathcal{Y}_{\infty}:=\varliminf_{n} \mathcal{Y}_{n}$. We have an inclusion $\mathcal{U}_{\infty} \subset \mathcal{Y}_{\infty}$. Class field theory gives

$$
0 \rightarrow \overline{\mathcal{E}}_{\infty} / \overline{\mathcal{C}}_{\infty} \rightarrow \mathcal{U}_{\infty} / \overline{\mathcal{C}}_{\infty} \rightarrow \mathcal{X}_{\infty} \rightarrow \mathcal{A}_{\infty} \rightarrow 0
$$

where $\overline{\mathcal{C}}_{\infty}$ is diagonally embedded into $\mathcal{U}_{\infty}^{\mathfrak{p}} \times \mathcal{U}_{\infty}^{\mathfrak{p}^{*}}$ if $p$ is split. On this sequence acts $\mathcal{G}$ and we get: 


\section{Corollary 2.1.5.}

$$
\operatorname{det}_{\Lambda \chi}\left(\mathcal{X}_{\infty}^{\chi}\right)=\operatorname{det}_{\Lambda \chi}\left(\mathcal{U}_{\infty}^{\chi} / \overline{\mathcal{C}}_{\infty}^{\chi}\right)
$$

Lemma 2.1.6. Let $p \nmid \mathrm{Nf}$ be a prime. If $p$ splits in $K$, the inclusion $\mathcal{U}_{\infty} \rightarrow$ $\mathcal{Y}_{\infty}$ is an isomorphism and if $p$ is inert or ramified in $K$, there is an exact sequence

$$
0 \rightarrow \mathcal{U}_{\infty} \rightarrow \mathcal{Y}_{\infty} \rightarrow \mathbb{Z}_{p}\left[\Delta / \Delta_{p}\right] \rightarrow 0
$$

where $\Delta_{p}$ is the decomposition group of $p$ in $\Delta=\operatorname{Gal}\left(K_{0} / K\right)$.

Proof. We have exact sequences

$$
1 \rightarrow \mathcal{U}_{n, v} \rightarrow K_{n . v}^{*} \rightarrow \mathbb{Z} \times \kappa_{n}^{*} \rightarrow 1
$$

where $\kappa_{n}$ is the residue class field of $K_{n . v}$. By definition $\mathcal{U}_{n}=\bigoplus_{v \mid p} \mathcal{U}_{n, v}$.

As the order of the residue class field $\kappa_{n}^{*}$ is prime to $p$, we have an exact sequence

$$
0 \rightarrow \lim _{n} \mathcal{U}_{n, v} / p^{n} \rightarrow \lim _{n} K_{n, v}^{*} / p^{n} \rightarrow \mathbb{Z}_{p} \rightarrow 0 .
$$

As $E$ has good reduction at $p$, we now how $p$ decomposes in $K_{n}$ (see Ru1 prop. 3.6). If $p$ is split, the ramification degree of $v$ in $K_{n+1}$ is $p$ and the degree of $K_{n+1}$ over $K_{n}$ is $p^{2}$. Hence the norm map induces multiplication by $p$ on $\mathbb{Z}_{p}$ and the inverse limit over these maps is zero. This gives the first claim. In the case where $p$ is inert of ramified, $v$ is totally ramified in $K_{n+1}$ and the norm map from $K_{n^{\prime}, v}^{*} \rightarrow K_{n, v}^{*}$ induces the identity on $\mathbb{Z}_{p}$. Putting these sequences together for all $v \mid p$ and using $\bigoplus_{v \mid p} \mathbb{Z}_{p}=\mathbb{Z}_{p}\left[\Delta / \Delta_{p}\right]$ gives the result.

\subsection{Reductions via Iwasawa theory}

In this section we use Rubin's "main conjecture" of Iwasawa theory to reduce the Bloch-Kato conjecture to a comparison between the space $\mathcal{R}_{\psi}$ of 1.2 .3 and the elliptic units $\overline{\mathcal{C}}_{\infty}$.

We have a subspace

$$
r_{p}\left(\mathcal{R}_{\psi}\right) \subset H^{1}\left(\mathcal{O}_{S}, V_{p}\right)
$$

where $\mathcal{O}_{S}=\mathcal{O}_{K}\left[\frac{1}{S}\right]$ and $V_{p}=T_{p} E(k+1) \otimes \mathbb{Q}_{p}$. Recall that $S$ is the set of primes of $K$ dividing $p \mathfrak{f}$. We want to compute the relation of the submodule 
$r_{p}\left(\mathcal{R}_{\psi}\right)$ to $H^{1}\left(\mathcal{O}_{S}, T_{p} E(k+1)\right)$. Our method, which is inspired by Kato's paper [Ka2], relates the module $r_{p}\left(\mathcal{R}_{\psi}\right)$ to a certain submodule defined by the elliptic units $\overline{\mathcal{C}}_{\infty}$. This submodule in turn is defined using an idea of Soulé.

Our aim is to relate the determinant of $\overline{\mathcal{C}}_{\infty} \otimes T_{p} E(k)$ to the determinant of $R \Gamma\left(\mathcal{O}_{S}, T_{p} E(k+1)\right)$ (see theorem 2.2.11 for the exact formulation).

In this section $p$ is always a prime which does not divide $\# \mathcal{O}_{K}^{*}$ and where $E$ has good reduction over the primes above $p$, i.e. $p \nmid \mathrm{N} \mathfrak{f}$.

Denote by abuse of notation by $S_{p}$ the set of primes over $p$ in the ring $\mathcal{O}_{n}$ for every $n$ and by $\mathcal{O}_{n, S_{p}}$ the ring of integers in $K_{n}$ where the primes above $p$ are inverted. We define $\mathcal{O}_{\infty, S_{p}}:=\varliminf_{n} \mathcal{O}_{n, S_{p}}$. Similarly we define $\mathcal{O}_{n, S}$ and $\mathcal{O}_{\infty, S}$

\subsubsection{Review of the Soulé elements}

We keep the notations from the section 2.1.

Denote by $T_{p} E=\varliminf_{n} E\left[p^{n}\right]$ the Tate module of $E$ and let $T_{p} E(k):=$ $T_{p} E \otimes \mathbb{Z}_{p}(k)$ its Tate twist. This is a $\mathcal{O}_{p} \otimes \mathbb{Z}_{p}[[\mathcal{G}]]$-module. We start by defining a map in the spirit of Soulé

$$
\overline{\mathcal{C}}_{\infty} \otimes_{\mathbb{Z}_{p}} T_{p} E(k) \rightarrow H^{1}\left(\mathcal{O}_{S}, T_{p} E(k+1)\right)
$$

here $T_{p} E(k+1)$ is a sheaf on $\mathcal{O}_{S}$ because it is unramified outside of $S$. Write

$$
H^{1}\left(\mathcal{O}_{S}, T_{p} E(k+1)\right)=\lim _{r} H^{1}\left(\mathcal{O}_{S}, E\left[p^{r+1}\right](k+1)\right)
$$

and let for a norm compatible system of elliptic units $\left(\theta_{r}\right)_{r}$ and an element $\left(t_{r}\right)_{r} \in T_{p} E(k+1)$

$$
e_{p}\left(\left(\theta_{r} \otimes t_{r}\right)_{r}\right):=\left(\mathrm{N}_{K_{r} / K}\left(\theta_{r} \otimes t_{r}\right)\right)_{r}
$$

where $\theta_{r} \otimes t_{r}$ is an element in

$$
\mathcal{O}_{r, S}^{*} /\left(\mathcal{O}_{r, S}^{*}\right)^{p^{r+1}} \otimes E\left[p^{r+1}\right](k) \subset H^{1}\left(\mathcal{O}_{r, S}, E\left[p^{r+1}\right](k+1)\right)
$$

(the inclusion comes from Kummer theory) and $\mathrm{N}_{K_{r} / K}$ is the norm map on the cohomology. According to Soule ( So2] lemma 1.4) this gives a projective system of elements in $H^{1}\left(\mathcal{O}_{S}, T_{p} E(k+1)\right)$. The map $e_{p}$ factors through the coinvariants under $\mathcal{G}$, so that we can make the following definition:

Definition 2.2.1. The Soulé elliptic elements are defined by the map

$$
e_{p}:\left(\overline{\mathcal{C}}_{\infty} \otimes T_{p} E(k)\right)_{\mathcal{G}} \rightarrow H^{1}\left(\mathcal{O}_{S}, T_{p} E(k+1)\right) .
$$

We want to investigate to what extend this map gives generators for $H^{1}\left(\mathcal{O}_{S}, T_{p} E(k+1)\right)$, i.e. what is the kernel and cokernel of the map $e_{p}$. 


\subsubsection{The Tate-Poitou localization sequence}

Our main tool in describing $H^{1}\left(\mathcal{O}_{S}, T_{p} E(k+1)\right)$ in terms of elliptic units will be the Tate-Poitou localization sequence. It is convenient for us to write down a derived category version of it.

For technical reasons we have to work first over $K_{0}$. The reason for this is that over $K_{0}$ the module $T_{p} E(k+1)$ is unramified outside of the primes above $p$ :

Lemma 2.2.2. (Ru1]1.3) If $p \nmid \# \mathcal{O}_{K}^{*}$, then over $K_{0}$ the elliptic curve $E$ has good reduction at all places not dividing $p$. In particular there exists a model of $E$ over $\mathcal{O}_{0, S_{p}}$ and $T_{p} E(k+1)$ is unramified.

The localization sequence now reads as follows (see [Ka2] (6.3)). Here ${ }^{*}$ is the Pontryagin dual $\operatorname{Hom}_{\mathcal{O}_{p}}\left(-, \mathbb{Q}_{p} / \mathbb{Z}_{p} \otimes_{\mathbb{Z}_{p}} \mathcal{O}_{p}\right)$.

$$
\begin{aligned}
R \Gamma\left(\mathcal{O}_{0, S_{p}}, T_{p} E(k+1)\right) \rightarrow & R \Gamma\left(K_{0} \otimes \mathbb{Q}_{p}, E\left[p^{\infty}\right](-k)\right)^{*}[-2] \\
& \rightarrow R \Gamma\left(\mathcal{O}_{0, S_{p}}, E\left[p^{\infty}\right](-k)\right)^{*}[-2] \rightarrow,
\end{aligned}
$$

where we have used the identification

$$
E\left[p^{\infty}\right](-k)=\operatorname{Hom}_{\mathcal{O}_{p}}\left(T_{p} E(k+1), \mathbb{Q}_{p} / \mathbb{Z}_{p}(1) \otimes \mathcal{O}_{p}\right) .
$$

Our next task is to rewrite this Tate-Poitou sequence in terms of Iwasawa theory.

\subsubsection{Identification of some Galois cohomology groups with Iwa- sawa modules}

Let us define

$$
\begin{aligned}
H^{1}\left(K_{\infty} \otimes \mathbb{Q}_{p}, E\left[p^{\infty}\right](-k)\right): & =\varliminf_{n} H^{1}\left(K_{n} \otimes \mathbb{Q}_{p}, E\left[p^{\infty}\right](-k)\right) \\
& =\varliminf_{n} \bigoplus_{v \mid p} H^{1}\left(K_{n, v}, E\left[p^{\infty}\right](-k)\right) .
\end{aligned}
$$

Note that there are only finitely many primes above $p$ in $K_{\infty}$.

Proposition 2.2.3. There are isomorphisms of $\mathcal{O}_{p}[[\mathcal{G}]]$-modules

$$
\begin{aligned}
& \mathcal{X}_{\infty} \otimes_{\mathbb{Z}_{p}} T_{p} E(k) \cong H^{1}\left(\mathcal{O}_{\infty, S_{p}}, E\left[p^{\infty}\right](-k)\right)^{*} \\
& \mathcal{Y}_{\infty} \otimes_{\mathbb{Z}_{p}} T_{p} E(k) \cong H^{1}\left(K_{\infty} \otimes \mathbb{Q}_{p}, E\left[p^{\infty}\right](-k)\right)^{*},
\end{aligned}
$$

where ${ }^{*}$ is the Pontryagin dual $\operatorname{Hom}_{\mathcal{O}_{p}}\left(-, \mathbb{Q}_{p} / \mathbb{Z}_{p} \otimes_{\mathbb{Z}_{p}} \mathcal{O}_{p}\right)$. 
Proof. We have

$$
\begin{aligned}
H^{1}\left(\mathcal{O}_{\infty, S_{p}}, E\left[p^{\infty}\right](-k)\right)^{*} & =\operatorname{Hom}\left(\operatorname{Gal}\left(\bar{K} / K_{\infty}\right), E\left[p^{\infty}\right](-k)\right)^{*} \\
& =\operatorname{Hom}\left(\operatorname{Gal}\left(M_{\infty}^{p} / K_{\infty}\right), E\left[p^{\infty}\right](-k)\right)^{*} \\
& =\mathcal{X}_{\infty} \otimes T_{p} E(k) .
\end{aligned}
$$

In the local case, we have an isomorphism

$$
\begin{aligned}
H^{1}\left(K_{n} \otimes \mathbb{Q}_{p}, E\left[p^{n}\right](-k)\right) & =\bigoplus_{v \mid p} H^{1}\left(K_{n, v}, E\left[p^{n}\right](-k)\right) \\
& =\bigoplus_{v \mid p} \operatorname{Hom}\left(\operatorname{Gal}\left(\bar{K}_{n, v} / K\right)^{\mathrm{ab}}, E\left[p^{n}\right](-k)\right)
\end{aligned}
$$

By class field theory

$$
\operatorname{Hom}\left(\operatorname{Gal}\left(\bar{K}_{n, v} / K\right)^{\mathrm{ab}}, E\left[p^{n}\right](-k)\right)^{*} \cong K_{n, v}^{*} / p^{n} \otimes E\left[p^{n}\right](k)
$$

so that

$$
H^{1}\left(K_{\infty} \otimes \mathbb{Q}_{p}, E\left[p^{\infty}\right](-k)\right)^{*}=\bigoplus_{v \mid p} \mathcal{Y}_{\infty} \otimes T_{p}(k)
$$

\subsubsection{Rewriting the Tate-Poitou localization sequence in terms of Iwasawa theory}

To proceed further, we need the following vanishing result.

Proposition 2.2.4. The groups

$$
H^{2}\left(K_{\infty} \otimes \mathbb{Q}_{p}, E\left[p^{\infty}\right](-k)\right) \quad \text { and } \quad H^{2}\left(\mathcal{O}_{\infty, S_{p}}, E\left[p^{\infty}\right](-k)\right)
$$

are zero.

Proof. By local duality we have

$$
H^{2}\left(K_{n} \otimes \mathbb{Q}_{p}, E\left[p^{\infty}\right](-k)\right)^{*} \cong H^{0}\left(K_{n} \otimes \mathbb{Q}_{p}, T_{p} E(k+1)\right)=0 .
$$

On the other hand it is a result of Schneider Sch1 4.1 that the cohomology group $H^{2}\left(\mathcal{O}_{\infty, S_{p}}, \mathbb{Q}_{p} / \mathbb{Z}_{p}(-k)\right)$ is zero. As

$$
H^{2}\left(\mathcal{O}_{\infty, S_{p}}, E\left[p^{\infty}\right](-k)\right)=H^{2}\left(\mathcal{O}_{\infty, S_{p}}, \mathbb{Q}_{p} / \mathbb{Z}_{p}(-k)\right) \otimes_{\mathbb{Z}_{p}} T_{p} E
$$

this proves our claim. 
This vanishing result implies that we get actually a map from the Iwasawa modules to complexes computing the Galois cohomology.

Corollary 2.2.5. There are exact triangles

$\mathcal{Y}_{\infty} \otimes T_{p} E(k)[1] \rightarrow R \Gamma\left(K_{\infty} \otimes \mathbb{Q}_{p}, E\left[p^{\infty}\right](-k)\right)^{*} \rightarrow H^{0}\left(K_{\infty} \otimes \mathbb{Q}_{p}, E\left[p^{\infty}\right](-k)\right)^{*}$

$\mathcal{X}_{\infty} \otimes T_{p} E(k)[1] \rightarrow R \Gamma\left(\mathcal{O}_{\infty, S_{p}}, E\left[p^{\infty}\right](-k)\right)^{*} \rightarrow H^{0}\left(\mathcal{O}_{\infty, S_{p}}, E\left[p^{\infty}\right](-k)\right)^{*}$

Proof. The propositions 2.2.3 and 2.2.4 show that we have a canonical map from $\mathcal{Y}_{\infty} \otimes T_{p} E(k)[1]$ to $R \Gamma\left(K_{\infty} \otimes \mathbb{Q}_{p}, E\left[p^{\infty}\right](-k)\right)^{*}$ because the second cohomology vanishes. The same argument gives the result for $\mathcal{X}_{\infty} \otimes T_{p} E(k)[1]$.

To relate these groups to the cohomology groups of $\mathcal{O}_{S_{p}}$ we want to take the coinvariants under $\Gamma=\operatorname{Gal}\left(K_{\infty} / K_{0}\right)$.

Lemma 2.2.6. Let $M$ be an perfect complex of $\Lambda=\mathcal{O}_{p}[[\Gamma]]$-modules. Then there are canonical isomorphisms

$$
M^{*} \otimes_{\Lambda}^{\mathbb{L}} \mathcal{O}_{p} \cong R \Gamma(\Gamma, M)^{*}
$$

where the right hand side is the (continuous) group cohomology of $\Gamma$ and $M^{*}=$ $\operatorname{Hom}\left(M, \mathbb{Q}_{p} / \mathbb{Z}_{p} \otimes \mathcal{O}_{p}\right)$.

Proof. We have

$$
\begin{aligned}
R \operatorname{Hom}_{\Lambda}\left(\mathcal{O}_{p}, M^{*}\right) & =R \operatorname{Hom}_{\Lambda}\left(\mathcal{O}_{p}, \operatorname{Hom}\left(M, \mathbb{Q}_{p} / \mathbb{Z}_{p} \otimes \mathcal{O}_{p}\right)\right) \\
& \left.=R \operatorname{Hom}_{\Lambda}\left(M \otimes \mathbb{L}_{\Lambda} \mathcal{O}_{p}, \mathbb{Q}_{p} / \mathbb{Z}_{p} \otimes \mathcal{O}_{p}\right)\right)
\end{aligned}
$$

which by biduality $M^{* *}=M$ proves our claim.

Corollary 2.2.7. There are exact triangles

$$
\begin{aligned}
\left(\mathcal{Y}_{\infty} \otimes T_{p} E(k)\right) \otimes \otimes_{\Lambda}^{\mathbb{L}} \mathcal{O}_{p} \rightarrow & R \Gamma\left(K_{0} \otimes \mathbb{Q}_{p}, E\left[p^{\infty}\right](-k)\right)^{*}[-1] \\
& \rightarrow R \Gamma\left(\Gamma, H^{0}\left(K_{\infty} \otimes \mathbb{Q}_{p}, E\left[p^{\infty}\right](-k)\right)\right)^{*}[-1]
\end{aligned}
$$

and

$$
\begin{aligned}
\left(\mathcal{X}_{\infty} \otimes T_{p} E(k)\right) \otimes_{\Lambda}^{\mathbb{L}} \mathcal{O}_{p} \rightarrow & R \Gamma\left(\mathcal{O}_{0, S_{p}}, E\left[p^{\infty}\right](-k)\right)^{*}[-1] \\
& \rightarrow R \Gamma\left(\Gamma, H^{0}\left(\mathcal{O}_{\infty, S_{p}},\left[p^{\infty}\right](-k)\right)\right)^{*}[-1]
\end{aligned}
$$

Proof. Apply lemma 2.2.6 to the exact triangles in corollary 2.2.5. 
Now we come back to the Tate-Poitou localization sequences over $\mathcal{O}_{0, S_{p}}$

$$
\begin{aligned}
R \Gamma\left(\mathcal{O}_{0, S_{p}}, T_{p} E(k+1)\right) \rightarrow & R \Gamma\left(K_{0} \otimes \mathbb{Q}_{p}, E\left[p^{\infty}\right](-k)\right)^{*}[-2] \\
& \rightarrow R \Gamma\left(\mathcal{O}_{0, S_{p}}, E\left[p^{\infty}\right](-k)\right)^{*}[-2] \rightarrow
\end{aligned}
$$

and the map $e_{p}$. Recall that

$$
e_{p}: \overline{\mathcal{C}}_{\infty} \otimes T_{p} E(k) \rightarrow H^{1}\left(\mathcal{O}_{S_{p}}, T_{p} E(k+1)\right)
$$

(see definition 2.2.1) and taking in the definition of $e_{p}$ only the norm maps to $K_{0}$ we get a map:

$$
e_{p}: \overline{\mathcal{C}}_{\infty} \otimes T_{p} E(k) \rightarrow H^{1}\left(\mathcal{O}_{0, S_{p}}, T_{p} E(k+1)\right)
$$

As $H^{0}\left(\mathcal{O}_{0, S_{p}}, T_{p} E(k+1)\right)=0$ for weight reasons, we get a map of complexes

$$
e_{p}:\left(\overline{\mathcal{C}}_{\infty} \otimes T_{p} E(k)\right) \otimes \mathbb{L}_{\Lambda}^{\mathbb{L}} \mathcal{O}_{p} \rightarrow R \Gamma\left(\mathcal{O}_{0, S_{p}}, T_{p} E(k+1)\right)[1] .
$$

This is compatible with the maps defined before:

Lemma 2.2.8. The following diagram is commutative

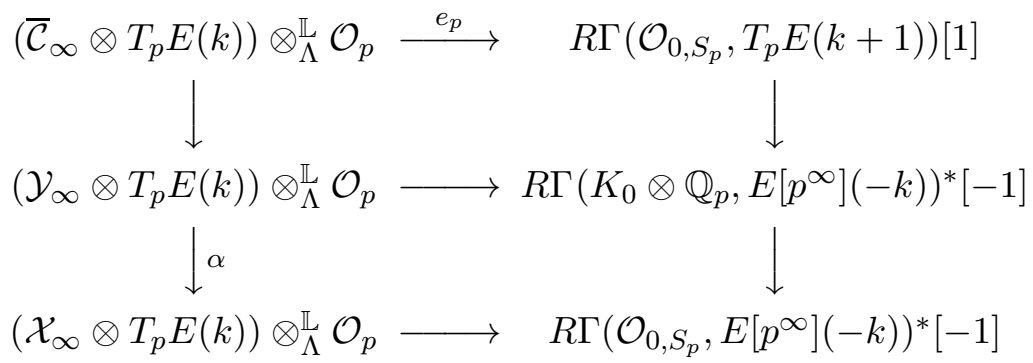

Here $\alpha$ is induced by the map $\mathcal{Y}_{\infty} / \overline{\mathcal{C}}_{\infty} \rightarrow \mathcal{X}_{\infty}$.

Proof. The commutativity of the lower square is clear. Let us treat the upper square. The map

$$
\left(\mathcal{Y}_{\infty} \otimes T_{p} E(k)\right)_{\Gamma} \rightarrow H^{1}\left(K_{0} \otimes \mathbb{Q}_{p}, E\left[p^{\infty}\right](-k)\right)^{*}
$$

is the dual of the corestriction

$$
H^{1}\left(K_{0} \otimes \mathbb{Q}_{p}, E\left[p^{\infty}\right](-k)\right) \rightarrow H^{1}\left(K_{\infty} \otimes \mathbb{Q}_{p}, E\left[p^{\infty}\right](-k)\right)^{\Gamma} .
$$

By the local duality theorem the corestriction map is dual to the norm map

$$
H^{1}\left(K_{\infty} \otimes \mathbb{Q}_{p}, T_{p} E(k+1)\right)_{\Gamma} \rightarrow H^{1}\left(K_{0} \otimes \mathbb{Q}_{p}, T_{p} E(k+1)\right) .
$$

This together with the definition of $e_{p}$ proves our claim. 


\subsubsection{The comparison theorem between elliptic units and Galois cohomology}

The next step is to relate the determinants of $\left(\overline{\mathcal{C}}_{\infty} \otimes T_{p} E(k)\right) \otimes \mathbb{L} \mathcal{O}_{p}$ and $R \Gamma\left(\mathcal{O}_{0, S_{p}}, T_{p} E(k+1)\right)$ as $\mathcal{O}_{p}[\Delta]$-modules. For this we need Rubin's "main conjecture". As the "main conjecture" is not proven for characters of $\Delta$, which are trivial on the decomposition group $\Delta_{p}$ of $p$, we need the following lemma:

Lemma 2.2.9. Let $p$ be inert or ramified, where $p$ is a prime over which $E$ has good reduction. Let $\chi$ be the $\Delta$ representation on $\operatorname{Hom}_{\mathcal{O}_{p}}\left(T_{p} E(k), \mathcal{O}_{p}\right)$. Then $\Delta_{p}=\Delta$ and $\chi$ is non trivial on $\Delta_{p}$.

Proof. Let $p$ be an inert or ramified prime and $\chi^{\prime}$ be the $\Delta$ representation on $T_{p} E$. Then $\chi^{\prime}$ is irreducible ( $\mathrm{Ru} 2$ 11.5.) and because $E$ has good reduction the prime above $p$ is totally ramified in $K_{0}$ (Ru1 3.6.) and $\Delta \cong(\mathcal{O} / \mathfrak{p})^{*}$. Now $\chi^{\prime}$ is two dimensional and $\chi$ is simply a twist of $\chi^{\prime}$ by a power of $\operatorname{det} \chi^{\prime}$. Thus $\chi$ acts non trivially on $\operatorname{Hom}_{\mathcal{O}_{p}}\left(T_{p} E(k), \mathcal{O}_{p}\right)$.

Corollary 2.2.10. Let $\chi$ be the $\Delta$ representation on $\operatorname{Hom}_{\mathcal{O}_{p}}\left(T_{p} E(k), \mathcal{O}_{p}\right)$ and $p \nmid \mathrm{N} \mathfrak{f}$ be a prime. Then

$$
\mathcal{U}_{\infty}^{\chi} \cong \mathcal{Y}_{\infty}^{\chi}
$$

Proof. If $p$ is split this follows immediately from lemma 2.1.6 and if $p$ is inert or prime in $K$ this follows from the same lemma and the above result because the $\chi$-eigenspace of $\mathcal{O}_{p}\left[\Delta / \Delta_{p}\right]$ is zero.

We can now formulate the main theorem of this section.

Theorem 2.2.11. Let $\chi$ be the $\Delta$-representation on $\operatorname{Hom}_{\mathcal{O}_{p}}\left(T_{p} E(k), \mathcal{O}_{p}\right)$ and assume that $p \nmid \mathrm{Nf}$. Then the map $e_{p}$ induces an isomorphism of $\mathcal{O}_{p}$-modules

$$
\operatorname{det}_{\mathcal{O}_{p}}\left(\left(\overline{\mathcal{C}}_{\infty}^{\chi} \otimes_{\mathcal{O}_{p}} T_{p} E(k)\right) \otimes_{\Lambda}^{\mathbb{L}} \mathcal{O}_{p}\right) \cong \operatorname{det}_{\mathcal{O}_{p}}\left(R \Gamma\left(\mathcal{O}_{S}, T_{p} E(k+1)\right)\right)^{-1}
$$

The rest of this section is concerned with the proof of this theorem. Let us first show:

Proposition 2.2.12. Let $\chi$ and $p$ be as in theorem 2.2.11, then

$$
\begin{aligned}
\operatorname{det}_{\mathcal{O}_{p}}\left(R \Gamma\left(\mathcal{G}, H^{0}\left(K_{\infty} \otimes \mathbb{Q}_{p}, E\left[p^{\infty}\right](-k)\right)\right)\right. & \cong \mathcal{O}_{p} \\
\operatorname{det}_{\mathcal{O}_{p}}\left(R \Gamma\left(\mathcal{G}, H^{0}\left(\mathcal{O}_{\infty, S_{p}}, E\left[p^{\infty}\right](-k)\right)\right)\right. & \cong \mathcal{O}_{p}
\end{aligned}
$$


Proof. The action of $\mathcal{G}$ on $T_{p}(k) \cong \mathcal{O}_{p}$ is via a character $\mathcal{G} \rightarrow \mathcal{O}_{p}^{*}$. This gives a surjection $\mathcal{O}_{p}[[\Gamma]] \rightarrow T_{p} E(k)$. As $\Gamma \cong \mathbb{Z}_{p}^{2}$ the kernel of this surjection is an ideal with height 2 and hence

$$
\operatorname{det}_{\mathcal{O}_{p}[[\mathcal{G}]]}\left(T_{p} E(k)\right) \cong \mathcal{O}_{p}[[\mathcal{G}]]
$$

This implies $\operatorname{det}_{\mathcal{O}_{p}}\left(T_{p} E(k) \otimes_{\mathcal{O}_{p}[[\mathcal{G}]]}^{\mathbb{L}} \mathcal{O}_{p}\right) \cong \mathcal{O}_{p}$. Lemma 2.2.6 then implies the claim.

Recall that by corollary 2.2 .10 we have an isomorphism

$$
\mathcal{U}_{\infty}^{\chi} \cong \mathcal{Y}_{\infty}^{\chi}
$$

Corollary 2.2.13. The triangles in corollary 2.2.7 give rise to isomorphisms

$\operatorname{det}_{\mathcal{O}_{p}}\left(\left(\mathcal{U}_{\infty}^{\chi} \otimes_{\mathcal{O}_{p}} T_{p} E(k)\right) \otimes \frac{\mathbb{L}}{\mathcal{O}_{p}}\right) \cong \operatorname{det}_{\mathcal{O}_{p}}\left(H^{0}\left(\Delta, R \Gamma\left(K_{0} \otimes \mathbb{Q}_{p}, E\left[p^{\infty}\right](-k)\right)^{*}[-1]\right)\right)$

$\operatorname{det}_{\mathcal{O}_{p}}\left(\left(\mathcal{X}_{\infty}^{\chi} \otimes_{\mathcal{O}_{p}} T_{p} E(k)\right) \otimes_{\Lambda}^{\mathbb{L}} \mathcal{O}_{p}\right) \cong \operatorname{det}_{\mathcal{O}_{p}}\left(H^{0}\left(\Delta, R \Gamma\left(\mathcal{O}_{0, S_{p}}, E\left[p^{\infty}\right](-k)\right)^{*}[-1]\right)\right)$

Proof. The complexes in the triangle in 2.2.7 are $\mathcal{O}_{p}[\Delta]$-modules and we apply $R \Gamma\left(\Delta,{ }_{-}\right)$. Then

$$
R \Gamma\left(\Delta, \mathcal{Y}_{\infty} \otimes_{\mathcal{O}_{p}} T_{p} E(k)\right) \cong \mathcal{Y}_{\infty}^{\chi} \otimes_{\mathcal{O}_{p}} T_{p} E(k)
$$

by definition of $\chi$. The same holds for $\mathcal{X}_{\infty} \otimes_{\mathcal{O}_{p}} T_{p} E(k)$. The result follows with proposition 2.2.12.

Corollary 2.2.14. There is an isomorphism of determinants

$$
\begin{aligned}
\operatorname{det}_{\mathcal{O}_{p}}\left(H^{0}(\Delta,\right. & \left.\left.R \Gamma\left(\mathcal{O}_{0, S_{p}}, T_{p} E(k+1)\right)\right)\right)^{-1} \cong \\
& \cong \operatorname{det}_{\mathcal{O}_{p}}\left(\left(\mathcal{U}_{\infty}^{\chi} \otimes_{\mathcal{O}_{p}} T_{p} E(k)\right) \otimes_{\Lambda}^{\mathbb{L}} \mathcal{O}_{p}\right) \operatorname{det}_{\mathcal{O}_{p}}\left(\mathcal{X}_{\infty}^{\chi} \otimes_{\mathcal{O}_{p}} T_{p} E(k) \otimes_{\Lambda}^{\mathbb{L}} \mathcal{O}_{p}\right)^{-1}
\end{aligned}
$$

Proof. Apply $R \Gamma\left(\Delta,{ }_{-}\right)$to the triangle

$$
\begin{aligned}
R \Gamma\left(\mathcal{O}_{0, S_{p}}, T_{p} E(k+1)\right) \rightarrow & R \Gamma\left(K_{0} \otimes \mathbb{Q}_{p}, E\left[p^{\infty}\right](-k)\right)^{*}[-2] \rightarrow \\
& \rightarrow R \Gamma\left(\mathcal{O}_{0, S_{p}}, E\left[p^{\infty}\right](-k)\right)^{*}[-2]
\end{aligned}
$$

and use the above corollary.

Finally, we need to investigate the relation of the cohomology of $\mathcal{O}_{0, S_{p}}$ and $\mathcal{O}_{0, S}$, which is the integral closure of $\mathcal{O}_{S}$ in $K_{0}$. 
Lemma 2.2.15. Let $p$ and $\chi$ be as in the theorem 2.2.11. The restriction map of cohomology of $\mathcal{O}_{0, S_{p}}$ to $\mathcal{O}_{0, S}$ induces an equality of determinants

$$
\begin{aligned}
\operatorname{det}_{\mathcal{O}_{p}}\left(H^{0}\left(\Delta, R \Gamma\left(\mathcal{O}_{0, S_{p}}, T_{p} E(k+1)\right)\right)\right) & \cong \operatorname{det}_{\mathcal{O}_{p}}\left(H^{0}\left(\Delta, R \Gamma\left(\mathcal{O}_{0, S}, T_{p} E(k+1)\right)\right)\right) \\
& \left.\cong \operatorname{det}_{\mathcal{O}_{p}}\left(R \Gamma\left(\mathcal{O}_{S}, T_{p} E(k+1)\right)\right)\right) .
\end{aligned}
$$

Proof. There is an exact triangle

$$
R \Gamma\left(\mathcal{O}_{0, S_{p}}, T_{p} E(k+1)\right) \rightarrow R \Gamma\left(\mathcal{O}_{0, S}, T_{p} E(k+1)\right) \rightarrow \bigoplus_{v \in S \backslash S_{p}} R \Gamma_{\kappa(v)}\left(\mathcal{O}_{v}, T_{p} E(k+1)\right)[1]
$$

where $\mathcal{O}_{v}$ is the local ring at $v$. As $T_{p} E(k+1)$ is unramified at the places $v$ in $K_{0}$, which are in $S \backslash S_{p}$ we have by purity

$$
R \Gamma_{\kappa(v)}\left(\mathcal{O}_{v}, T_{p} E(k+1)\right) \cong R \Gamma\left(\kappa(v), T_{p} E(k)\right) .
$$

Let us prove that

$$
H^{0}\left(\Delta, \bigoplus_{v \in S \backslash S_{p}} R \Gamma\left(\kappa(v), T_{p} E(k)\right)\right)=0 .
$$

For this note that $H^{1}\left(\kappa(v), T_{p} E(k)\right) \cong T_{p} E(k)_{\mathrm{Gal}(\overline{\kappa(v)} / \kappa(v))}$ are the coinvariants and that $H^{0}=0$. Fix a prime $v \in S \backslash S_{p}$ of $K$ dividing $\mathfrak{f}$, then the primes $v_{0} \mid v$ of $K_{0}$ are permuted by $\Delta$. Fix $v_{0}$ dividing $v$ and let $\Delta_{v_{0}}$ be the stabilizer of $v_{0}$. It suffices to prove that $\Delta_{v_{0}}$ acts non trivially on $T_{p} E(k)_{\operatorname{Gal}(\overline{\kappa(v)} / \kappa(v))}$. Let $I_{v_{0}} \subset \Delta_{v_{0}}$ be the inertia group of $v_{0}$. This group is non trivial because $K_{0} / K$ is ramified above $v$ by lemma 2.2.2 and it acts non trivially on $T_{p} E(k)$ because $v_{0} \mid \mathfrak{f}$ and by the Neron-Ogg-Shavarevich criterium. This proves our claim.

Now we can prove the theorem.

Proof. (of theorem 2.2.11) Let $\chi$ and $p$ be as in the theorem. By Rubin's "main conjecture" 2.1.5 we have

$$
\begin{aligned}
\operatorname{det}_{\mathcal{O}_{p}}\left(\left(\mathcal{U}_{\infty}^{\chi} / \overline{\mathcal{C}}_{\infty}^{\chi}\right) \otimes T_{p} E(k) \otimes_{\Lambda}^{\mathbb{L}} \mathcal{O}_{p}\right) & \cong \operatorname{det}_{\Lambda}\left(\left(\mathcal{U}_{\infty}^{\chi} / \overline{\mathcal{C}}_{\infty}^{\chi}\right) \otimes T_{p} E(k)\right) \otimes_{\Lambda} \mathcal{O}_{p} \\
& \cong \operatorname{det}_{\Lambda}\left(\mathcal{X}_{\infty}^{\chi} \otimes T_{p} E(k)\right) \otimes_{\Lambda} \mathcal{O}_{p} \\
& \cong \operatorname{det}_{\mathcal{O}_{p}}\left(\mathcal{X}_{\infty}^{\chi} \otimes T_{p} E(k) \otimes_{\Lambda}^{\mathbb{L}} \mathcal{O}_{p}\right)
\end{aligned}
$$

On the other hand,

$$
\operatorname{det}_{\Lambda \chi}\left(\mathcal{U}_{\infty}^{\chi} / \overline{\mathcal{C}}_{\infty}^{\chi}\right) \cong \operatorname{det}_{\Lambda \chi}\left(\mathcal{U}_{\infty}^{\chi}\right) \otimes \operatorname{det}_{\Lambda \chi}\left(\overline{\mathcal{C}}_{\infty}^{\chi}\right)^{-1}
$$

This together with the above corollaries gives the result. 


\section{The elliptic polylogarithm sheaf}

We start afresh with the aim of computing the specialization of the elliptic polylog. For this we have to recall the definition of the polylogarithm sheaf and give a geometric interpretation of it.

We review here mostly Beilinson and Levin [Be-Le]. Everything that follows will be in the general setting of an elliptic curve over any base $S$. Because of this we start with fixing the notations. Then we review the unipotent elliptic polylog of Beilinson and Levin. For our geometrical construction we need a different description of this polylogarithm sheaf in terms of the fundamental group of the elliptic curve. This description is in section 3.3. The comparison of these two approaches will be carried out in section 3.4. Finally we consider the specialization of the polylogarithm sheaf at torsion points. This gives the $l$-adic Eisenstein classes.

\subsection{Notations and conventions}

Let $S$ be a scheme, and $l$ be a prime number invertible on $S$. We fix a base ring $\Lambda:=\mathbb{Z} / l^{r} \mathbb{Z}, \mathbb{Z}_{l}$ or $\mathbb{Q}_{l}$. In this section we introduce some notations for elliptic curves over $S$ and for pro- $\Lambda$-sheaves.

\subsubsection{Elliptic curves and coverings}

Definition 3.1.1. An elliptic curve is a smooth proper morphism $\bar{\pi}: E \rightarrow S$ together with a section $e: S \rightarrow E$, such that the geometric fibers $E_{\bar{s}}$ of $\bar{\pi}$ are connected curves of genus 1.

We introduce the following notation: On $E$ we have the multiplication by $N$ map, which we denote by $[N]$. We let $H_{n}:=\operatorname{ker}\left[l^{n}\right]$ and we denote by $E_{n}$ the curve $E$ over $S$ considered as a $H_{n}$-torsor over $E$. The $l^{n}$-multiplication map will then be denoted by $p_{n}: E_{n} \rightarrow E$. Let $U_{n}:=E_{n} \backslash H_{n}$ and $U:=E \backslash e(S)$, so that we have a Cartesian diagram

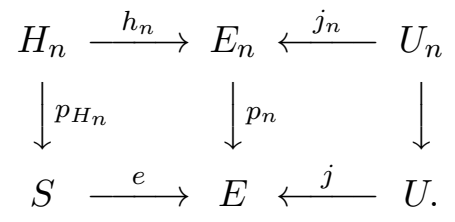

The unit section of $E_{n}$ will be $e_{n}$, if confusion is likely. The map $E_{m} \rightarrow E_{n}$ for $m \geq n$, which is the multiplication by $l^{m-n}$, is denoted by $p_{m, n}$ or even $p$. Let $\bar{\pi}_{n}: E_{n} \rightarrow S$ and $\pi_{n}: U_{n} \rightarrow S$ be the structure maps. 


\subsubsection{Pro-sheaves}

The polylogarithm is an extension of pro-sheaves and we will work in the category of pro-sheaves. For convenience of the reader we recall the definition and the main properties of pro-objects in the case we need.

Let $\mathcal{A}$ be an abelian category.

Definition 3.1.2. The category pro-A of pro-objects is the category whose objects are projective systems

$$
A: I^{\mathrm{op}} \rightarrow \mathcal{A}
$$

denoted by $\left(A_{i}\right)_{i \in I}$, where $I$ is some small filtered index category. The morphisms are

$$
\operatorname{Hom}_{\text {pro }-\mathcal{A}}\left(\left(A_{i}\right),\left(B_{j}\right)\right):=\lim _{j} \underline{\lim }_{i} \operatorname{Hom}_{\mathcal{A}}\left(A_{i}, B_{j}\right) .
$$

The category pro $-\mathcal{A}$ is again abelian (see Ar-Ma A 4.5). We call an object $\left(A_{i}\right)_{I} \in$ pro-A Mittag-Leffler zero if for every $i \in I$ there is an $i \rightarrow j$ such that $A_{j} \rightarrow A_{i}$ is the zero map. An element is zero in pro $-\mathcal{A}$ if and only if it is Mittag-Leffler zero (see Ar-Ma A 3.5). A functor $F: \mathcal{A} \rightarrow \mathcal{B}$ is extended to the pro-categories in the obvious way $F\left(\left(\mathcal{F}_{i}\right)_{i}\right):=\left(F\left(\mathcal{F}_{i}\right)\right)_{i}$.

Let us specialize to the category $\operatorname{Sh}(X)$ of étale sheaves on a scheme $X$. We denote by pro $-\operatorname{Sh}(X)$ the associated category of pro-sheaves as defined above. Pro-sheaves will usually be written as $\left(\mathcal{F}_{i}\right)_{i}$ the transition maps understood. For two pro-sheaves $\left(\mathcal{F}_{i}\right)_{i}$ and $\left(\mathcal{G}_{i}\right)_{i}$ on a scheme $X$ define $\operatorname{Ext}_{X}^{j}\left(\left(\mathcal{F}_{i}\right)_{i},\left(\mathcal{G}_{i}\right)_{i}\right)$ to be the group of $j$-th Yoneda extensions of $\left(\mathcal{F}_{i}\right)_{i}$ by $\left(\mathcal{G}_{i}\right)_{i}$ in pro $-\operatorname{Sh}(X)$.

\subsection{Review of the elliptic polylogarithm}

We first recall the definition of the elliptic logarithm sheaf from Be-Le]. Then we define the elliptic polylogarithmic sheaf.

\subsubsection{The unipotent logarithm sheaf}

Recall that $\Lambda$ is either $\mathbb{Z} / l^{r} \mathbb{Z}, \mathbb{Z}_{l}$ or $\mathbb{Q}_{l}$.

Definition 3.2.1. A lisse $\Lambda$-sheaf $\mathcal{F}$ on $E$ is unipotent of length $n$, if it admits a filtration $\mathcal{F}=\mathcal{F}^{0} \supset \mathcal{F}^{1} \supset \ldots \supset \mathcal{F}^{n} \supset 0$ such that $\mathrm{Gr}^{i} \mathcal{F}=\bar{\pi}^{*} \mathcal{G}^{i}$ for some lisse $\Lambda$-sheaf $\mathcal{G}^{i}$ on $S$.

Let $\mathcal{H}_{\Lambda}:=\underline{\operatorname{Hom}}_{S}\left(R^{1} \bar{\pi}_{*} \Lambda, \Lambda\right)$, then the boundary map for the exact se-

quence $0 \rightarrow \mathrm{Gr}^{i+1} \mathcal{F} \rightarrow \mathcal{F}^{i} / \mathcal{F}^{i+2} \rightarrow \mathrm{Gr}^{i} \mathcal{F} \rightarrow 0$ induces by duality a map

$$
\mathcal{H}_{\Lambda} \otimes \operatorname{Gr}^{i} \mathcal{F} \rightarrow \mathrm{Gr}^{i+1} \mathcal{F} \text {. }
$$


This gives an action of the ring $S \leq n:=\bigoplus_{k=0}^{n} \operatorname{Sym}^{k} \mathcal{H}_{\Lambda}$ on $\mathrm{Gr}^{\bullet} \mathcal{F}$. Beilinson and Levin prove:

Theorem 3.2.2. (Be-Le 1.2.6.) There is a k-unipotent sheaf $\mathcal{L}_{\log }{ }^{(k)}$ together with a section $1^{(k)}: \Lambda \rightarrow e^{*} \log ^{(k)}$ of the fibre at the unit section $e$ of $E$, which is unique up to isomorphism, such that for every $k$-unipotent sheaf $\mathcal{F}$ the map

$$
\begin{aligned}
& \bar{\pi}_{* \underline{\operatorname{Hom}}_{E}}(\mathcal{L} \operatorname{og}(n), \mathcal{F}) \rightarrow e^{*} \mathcal{F} \\
& f \mapsto f \circ 1^{(n)}
\end{aligned}
$$

is an isomorphism.

Recall also from Be-Le] that this is equivalent to the fact that the map $\nu: S \leq k \rightarrow \bar{\pi}_{*} \mathrm{Gr}^{\bullet} \mathcal{L} \mathrm{og}^{(k)}$ that sends 1 to $1^{(n)}$ is an isomorphism.

Definition 3.2.3. The canonical maps $\mathcal{L o g}^{(k+1)} \rightarrow \mathcal{L}{ }^{(k)}$ that map $1^{(k+1)}$ to $1^{(k)}$ make

$$
\mathcal{L} \operatorname{og}:=\left(\mathcal{L}^{(k)}\right)_{k}
$$

a pro-sheaf, which is called the logarithm sheaf. If it is necessary to indicate $\Lambda$ we write $\mathcal{L}^{(k)}{ }_{\Lambda}$ and $\log _{\Lambda}$.

Denote by $\mathcal{R}^{(k)}:=e^{*} \mathcal{L}{ }^{(k)}$ the fibre of $\mathcal{L}{ }^{(k)}$. This is a ring with identity given by $1^{(k)}$. Moreover $\mathcal{R}:=e^{*} \mathcal{L}$ og has a Hopf algebra structure. Then $\bar{\pi}^{*} \mathcal{R}^{(k)}$ acts on $\mathcal{L}_{\operatorname{og}}{ }^{(k)}$ and for every section $t: S \rightarrow E$ the sheaf $t^{*} \mathcal{L}_{\operatorname{og}}{ }^{(k)}$ is a free module of rank 1 over $\mathcal{R}^{(k)}$. The action of $\bar{\pi}^{*} \mathcal{R}^{(k)}$ on $\mathcal{L}_{\mathrm{og}}{ }^{(k)}$ induces via the isomorphism $\bar{\pi}_{*} \underline{\operatorname{Hom}}_{E}\left(\mathcal{L}_{\mathrm{og}}{ }^{(k)}, \mathcal{F}\right) \stackrel{\sim}{\rightarrow} e^{*} \mathcal{F}$ an action of $\mathcal{R}^{(k)}$ on $e^{*} \mathcal{F}$. In fact we have:

Proposition 3.2.4. (Be-L] 1 .2.10v)) The map $\mathcal{F} \mapsto e^{*} \mathcal{F}$ is an equivalence of the category of $k$-unipotent sheaves on $E$ with the category of lisse $\mathcal{R}^{(k)}$ modules on $S$.

We just remark that the inverse functor is $\mathcal{M} \mapsto \bar{\pi}^{*} \mathcal{M} \otimes_{\bar{\pi}^{*} \mathcal{R}^{(k)}} \mathcal{L} \operatorname{og}{ }^{(k)}$.

\subsubsection{Higher direct images of the logarithm sheaf}

Denote by $\mathcal{I}^{(k)}$ the augmentation ideal of the $\operatorname{ring} \mathcal{R}^{(k)}$. The pro-sheaves $\left(\mathcal{R}^{(k)}\right)_{k}$ and $\left(\mathcal{I}^{(k)}\right)_{k}$ are denoted by $\mathcal{R}$ and $\mathcal{I}$ respectively. The important fact for the definition of the polylogarithm is the computation of the higher direct images of $\mathcal{L}_{\mathrm{og}}{ }^{(k)}$. 
Proposition 3.2.5. (see $B e-L]$ 1.2.7) The higher direct images of $\mathcal{L o g}_{\Lambda}^{(k)}$ are

$$
R^{i} \bar{\pi}_{*} \mathcal{L}_{\operatorname{og}_{\Lambda}}^{(k)}=\left\{\begin{array}{lll}
\operatorname{Sym}^{k} \mathcal{H}_{\Lambda} & \text { if } & i=0 \\
\operatorname{Sym}^{k+1} \mathcal{H}_{\Lambda}(-1) & \text { if } & i=1 \\
\Lambda(-1) & \text { if } & i=2
\end{array}\right.
$$

The transition maps $R^{i} \bar{\pi}_{*} \mathcal{L}_{\operatorname{og}}{ }^{(k+1)} \rightarrow R^{i} \bar{\pi}_{*} \mathcal{L}_{\operatorname{og}}{ }^{(k)}$ are zero for $i=0,1$ and the identity for $i=2$. In particular $R^{i} \bar{\pi}_{*} \mathcal{L} \mathrm{og}=0$ for $i=0,1$ and $R^{2} \bar{\pi}_{*} \mathcal{L} \mathrm{og}=$ $\Lambda(-1)$.

For all the properties of the logarithm sheaf we refer to section 1.2. in Be-La].

Remark: Note that in the case of $\Lambda=\mathbb{Q}_{l}$ we have an isomorphism $\log ^{(k)} \cong$ $\operatorname{Sym}^{k} \mathcal{L}_{\mathrm{og}}{ }^{(1)}$ which sends $1^{(k)}$ to $1^{(1) k} / k$ !. This approach to the logarithm sheaf is used in $\mathrm{Hu}-\mathrm{Ki} 2]$.

Recall that $U:=E \backslash e$ is the complement of the unit section and $\pi: U \rightarrow S$ its structure map.

Proposition 3.2.6. The pro-sheaves $\left(R^{i} \pi_{*} \mathcal{L} \operatorname{og}{ }^{(k)}\right)_{k}$ are Mittag-Leffler zero for $i \neq 1$ and the canonical map

$$
R^{1} \pi_{*} \mathcal{L}^{(k)} \rightarrow e^{*} \log ^{(k)}(-1)=\mathcal{R}^{(k)}(-1)
$$

induces an isomorphism of pro-sheaves $\left(R^{1} \pi_{*} \mathcal{L}_{\mathrm{og}}(k)(1)\right)_{k} \cong\left(\mathcal{I}^{(k)}\right)_{k}$.

Proof. Consider the localization sequence

$$
\rightarrow R^{i} \bar{\pi}_{*} \mathcal{L o g}^{(k)} \rightarrow R^{i} \pi_{*} \mathcal{L}^{(k)} \rightarrow R^{i+1} e^{!} \mathcal{L}^{(k)}{ }^{(k)} \rightarrow
$$

and the purity isomorphism $R^{2} e^{!} \mathcal{L}_{\operatorname{og}}{ }^{(k)}=e^{*} \mathcal{L}_{\operatorname{og}}{ }^{(k)}(-1)$. Moreover $R^{i} e^{!} \mathcal{L}_{\operatorname{og}}{ }^{(k)}=$ 0 for $i \neq 2$. This together with the above values of $R^{i} \bar{\pi}_{*} \mathcal{L}$ og gives the desired result.

\subsubsection{The polylogarithm sheaf}

We are now going to define the elliptic polylogarithm. We will not use the usual approach using an identification of an Ext with a Hom-group but an other direct construction due to Beilinson and Levin [Be-Le] 1.3.6. This has the advantage of giving directly a pro-sheaf and not only an extension class. Moreover this sheaf can be easily compared to the geometric construction we give later. 
For every sheaf $\mathcal{F}$ on $E$ we denote by $\mathcal{F}_{U}$ its restriction to $U$. Let $\mathcal{F}$ be a lisse $\Lambda$-sheaf on $E$ and consider the open immersion

$$
j: U \times{ }_{S} U \backslash \Delta \hookrightarrow U \times{ }_{S} U
$$

where $\Delta$ is the diagonal. Define a lisse $\Lambda$-sheaf $H_{e}(\mathcal{F})$ on $U$ as follows:

Definition 3.2.7. Define a functor from lisse $\Lambda$-sheaves on $E$ to lisse $\Lambda$ sheaves on $U$ by

$$
H_{e}(\mathcal{F}):=R^{1} \operatorname{pr}_{1 *} j ! \operatorname{pr}_{2}^{*} \mathcal{F}_{U},
$$

where $\mathrm{pr}_{2}$ is the projection of $U \times{ }_{S} U \backslash \Delta$ to the second factor.

The exact sequence

$$
0 \rightarrow j ! \operatorname{pr}_{2}^{*} \mathcal{F}_{U} \rightarrow \operatorname{pr}_{2}^{*} \mathcal{F}_{U} \rightarrow \Delta_{*} \mathcal{F}_{U} \rightarrow 0
$$

induces an exact sequence

$$
0 \rightarrow \pi^{*} \bar{\pi}_{*} \mathcal{F} \rightarrow \mathcal{F}_{U} \stackrel{\alpha}{\rightarrow} H_{e}(\mathcal{F}) \rightarrow \pi^{*} R^{1} \pi_{*} \mathcal{F}_{U} \rightarrow 0 .
$$

Obviously this sequence is functorial in $\mathcal{F}$ so that we have the same sequence for pro-sheaves $\left(\mathcal{F}_{k}\right)_{k}$.

Lemma 3.2.8. The sequence (5) induces an exact sequence of pro-sheaves

$$
0 \rightarrow \mathcal{L} \operatorname{og}(1)_{U} \rightarrow H_{e}(\mathcal{L} \operatorname{og}(1)) \rightarrow \pi^{*} \mathcal{I} \rightarrow 0 .
$$

Proof. By proposition 3.2.6 we have $\bar{\pi}_{*} \mathcal{L} \operatorname{og}(1)=0$ and $R^{1} \pi_{*} \mathcal{L} \operatorname{og}(1) \cong \mathcal{I}$. This implies the claim.

Thus $H_{e}(\mathcal{L} \operatorname{og}(1))$ gives a class in $\operatorname{Ext}_{U}^{1}\left(\pi^{*} \mathcal{I}, \mathcal{L} \operatorname{og}(1)_{U}\right)$ which was defined in section 3.1 .2 as the group of Yoneda extensions in the abelian category pro $-\operatorname{Sh}(U)$. We have $H_{e}\left(\mathcal{L} \operatorname{og}(1) \otimes \pi^{*} \mathcal{R}\right) \cong H_{e}(\mathcal{L} \operatorname{og}(1)) \otimes \pi^{*} \mathcal{R}$ so that the action of $\pi^{*} \mathcal{R}$ on $\mathcal{L}$ og gives a $\pi^{*} \mathcal{R}$-module structure on $H_{e}(\mathcal{L} \operatorname{og}(1))$. In particular $H_{e}(\mathcal{L} \operatorname{og}(1))$ is a class in $\operatorname{Ext}_{U, \pi^{*} \mathcal{R}}^{1}\left(\pi^{*} \mathcal{I}, \mathcal{L} \operatorname{og}(1)_{U}\right)$, i.e. a Yoneda extension of $\pi^{*} \mathcal{R}$-modules.

Definition 3.2.9. The pro-sheaf

$$
\mathcal{P} \text { ol }:=H_{e}(\mathcal{L} \operatorname{og}(1))
$$

is the elliptic polylogarithm sheaf. If we need to indicate the dependence on $\Lambda$ we write $\mathcal{P}_{\mathrm{ol}}$. We also define $\mathcal{P}_{\mathrm{ol}}{ }^{(k)}:=H_{e}\left(\mathcal{L}_{\mathrm{og}}{ }^{(k)}(1)\right)$. 


\subsection{A geometric approach to the elliptic polylog sheaf}

We now present a different construction of the logarithm sheaf in the case $\Lambda=\mathbb{Z} / l^{r} \mathbb{Z}$. This makes explicit the remark in Be-Le 1.2.5.

\subsubsection{The geometric logarithm sheaf}

Recall that $p_{n}=\left[l^{n}\right]: E_{n} \rightarrow E$ and consider the sheaves

$$
\mathcal{L}_{\log _{n}^{\mathrm{g}}}:=p_{n *} \Lambda
$$

on $E$. For $m \geq n$ we have the trace map $p_{m_{*}} \Lambda \rightarrow p_{n_{*}} \Lambda$ and we define:

Definition 3.3.1. The geometric logarithm sheaf is the pro-sheaf

$$
\mathcal{L}^{\mathrm{g}} \mathrm{g}:=\left(\mathcal{L}_{\operatorname{og}_{n}^{\mathrm{g}}}^{\mathrm{g}}\right)_{n}
$$

where the transition maps are the above trace maps. Let

$$
\mathcal{R}^{\mathrm{g}}:=\left(\mathcal{R}_{n}^{\mathrm{g}}\right)_{n}:=\left(e^{*} \mathcal{L}_{\mathrm{og}_{n}^{\mathrm{g}}}^{\mathrm{g}}\right)_{n}
$$

be the pro-sheaf defined by the pull-back of $\mathcal{L}_{\mathrm{og}_{n}^{\mathrm{g}}}$ along the unit section e. Let $\mathcal{I}^{\mathrm{g}}:=\left(\mathcal{I}_{n}^{\mathrm{g}}\right)_{n}:=\operatorname{ker}\left(\mathcal{R}^{\mathrm{g}} \rightarrow \Lambda\right)$ be the augmentation ideal of $\mathcal{R}^{\mathrm{g}}$.

Note that the existence of the section $e_{n}$ of $H_{n}=p_{n}^{-1}(e)$ implies that there is a map $1_{n}: \Lambda \rightarrow e^{*} \mathcal{L}_{0_{n}}^{\mathrm{g}}=\mathcal{R}_{n}^{\mathrm{g}}$. The action of $H_{n}$ on $E_{n}$ over $E$ gives an action of $H_{n}$ on $\mathcal{L}_{\operatorname{og}_{n}^{\mathrm{g}}}^{\mathrm{g}}$, hence an action of $\bar{\pi}^{*} \mathcal{R}_{n}^{\mathrm{g}}$ on $\mathcal{L}_{\mathrm{og}_{n}^{\mathrm{g}}}^{\mathrm{g}}$.

The sheaf $\mathcal{L}_{g_{n}}^{\mathrm{g}}$ has the following important property. Recall that $\Lambda=$ $\mathbb{Z} / l^{r} \mathbb{Z}$

Proposition 3.3.2. For every lisse $\Lambda$-sheaf $\mathcal{F}$ the map

$$
\begin{aligned}
\stackrel{\lim }{\longrightarrow}_{n} \bar{\pi}_{*} \underline{\operatorname{Hom}}_{E}\left(\mathcal{L}_{\mathrm{Oog}_{n}^{\mathrm{g}}}^{\mathrm{g}}, \mathcal{F}\right) & \rightarrow e^{*} \mathcal{F} \\
f & \mapsto f \circ 1_{n}
\end{aligned}
$$

is an isomorphism.

Proof. The map $p_{n}$ is finite étale, so that

$$
\underline{\operatorname{Hom}}_{E}\left(p_{n *} \Lambda, \mathcal{F}\right)=\underline{\operatorname{Hom}}_{E_{n}}\left(\Lambda, p_{n}^{*} \mathcal{F}\right)=p_{n}^{*} \mathcal{F} .
$$

As $\mathcal{F}$ is a lisse $\Lambda$-sheaf, there is an $n$ such that $p_{n}^{*} \mathcal{F}$ comes from $S$, i.e. $p_{n}^{*} \mathcal{F} \cong$ $\bar{\pi}_{n}^{*} e_{n}^{*} p_{n}^{*} \mathcal{F}$. Thus

$$
\underline{\lim }_{n} \bar{\pi}_{*} \underline{\operatorname{Hom}}_{E}\left(\mathcal{L}_{\operatorname{og}_{n}^{\mathrm{g}}}^{\mathrm{g}}, \mathcal{F}\right)=\underline{\lim }_{n} \bar{\pi}_{n *} \bar{\pi}_{n}^{*} e_{n}^{*} p_{n}^{*} \mathcal{F}=\underline{\lim }_{n} e_{n}^{*} p_{n}^{*} \mathcal{F}=e^{*} \mathcal{F}
$$

which proves our claim. 
Let $\mathcal{F}$ be a lisse $\Lambda$-sheaf, then the action of $\mathcal{R}_{n}^{\mathrm{g}}$ on $\mathcal{L}_{\operatorname{og}_{n}^{\mathrm{g}}}$ induces via the above proposition an action of $\mathcal{R}_{n}^{\mathrm{g}}$ on $e^{*} \mathcal{F}$ for some $n$.

Corollary 3.3.3. The functor $\mathcal{F} \mapsto e^{*} \mathcal{F}$ induces an equivalence of the category of lisse $\Lambda$-modules on $E$ and lisse $\Lambda$-modules on $S$ with a continuous action of the pro-sheaf $\left(\mathcal{R}_{n}^{\mathrm{g}}\right)_{n}$ (i.e. the action factors through $\mathcal{R}_{m}^{\mathrm{g}}$ for some $m)$.

Proof. The inverse functor is given by

$$
\mathcal{M} \mapsto \bar{\pi}^{*} \mathcal{M} \otimes_{\bar{\pi}^{*} \mathcal{R}_{m}^{\mathrm{g}}} \mathcal{L} \log _{m}^{\mathrm{g}}
$$

if the action of $\left(\mathcal{R}_{n}^{\mathrm{g}}\right)_{n}$ factors through $\mathcal{R}_{m}^{\mathrm{g}}$.

\subsubsection{The higher direct images of the geometric logarithm sheaf}

As for $\mathcal{L}$ og we can compute the higher direct images of $\mathcal{L}_{\mathrm{og}}{ }^{\mathrm{g}}$ :

Lemma 3.3.4. The pro-sheaf

$$
\left(R^{i} \bar{\pi}_{*} \mathcal{L}_{\operatorname{og}_{n}^{\mathrm{g}}}^{\mathrm{g}}\right)_{n}
$$

is Mittag-Leffler zero for $i \neq 2$ and

$$
\left(R^{2} \bar{\pi}_{*} \mathcal{L}_{\operatorname{og}_{n}^{\mathrm{g}}}^{\mathrm{g}}\right)_{n} \cong \Lambda(-1) .
$$

Proof. We have to compute the transition maps in

$$
\left(R^{i} \bar{\pi}_{*} p_{n *} \Lambda\right)_{n}=\left(R^{i} \bar{\pi}_{n *} \Lambda\right)_{n} \cong\left(R^{i} \bar{\pi}_{*} \Lambda\right)_{n}
$$

where now the transition maps $R^{i} \bar{\pi}_{*} \Lambda \rightarrow R^{i} \bar{\pi}_{*} \Lambda$ are given by multiplication with $\left(l^{m-n}\right)^{2-i}$. This map is zero for $m \geq n+r$.

Corollary 3.3.5. The pro-sheaves $\left(R^{i} \pi_{*} \mathcal{L}_{\operatorname{og}_{n}^{\mathrm{g}}}^{\mathrm{g}}\right)_{n}$ are Mittag-Leffler zero for $i \neq 1$ and the canonical map

$$
R^{1} \pi_{*} \mathcal{L}_{\operatorname{og}_{n}^{\mathrm{g}}}^{\mathrm{g}} \rightarrow e^{*} \mathcal{L}_{\log _{n}^{\mathrm{g}}}^{\mathrm{g}}(-1)=\mathcal{R}_{n}^{\mathrm{g}}
$$

induces an isomorphism of pro-sheaves $\left(R^{1} \pi_{*} \mathcal{L}_{\operatorname{og}_{n}^{\mathrm{g}}}\right)_{n} \cong\left(\mathcal{I}_{n}^{\mathrm{g}}\right)_{n}$.

Proof. See the proof of 3.2 .6 . 


\subsubsection{The geometric polylogarithm sheaf}

The geometric polylog sheaf can now be defined in the same way as in 3.2.9. Recall the functor $H_{e}$ from definition 3.2.7.

Definition 3.3.6. The geometric elliptic polylog sheaf is the pro-sheaf

$$
\mathcal{P}_{\mathrm{ol}}^{\mathrm{g}}:=H_{e}\left(\mathcal{L}_{\operatorname{og}^{\mathrm{g}}}(1)\right)
$$

To indicate the dependence on $\Lambda$ we write $\mathcal{P}_{\mathrm{ol}}^{\mathrm{g}}$ and we define

$$
\mathcal{P}_{n}^{\mathrm{g}}:=H_{e}\left(\mathcal{L}_{n}^{\mathrm{g}}(1)\right) .
$$

As before the pro-sheaf $\mathcal{P}_{\mathrm{ol}^{\mathrm{g}}}$ is a sheaf of $\pi^{*} \mathcal{R}^{\mathrm{g}}$-modules and defines a Yoneda extension class in

$$
\operatorname{Ext}_{U, \pi^{*} \mathcal{R}^{\mathrm{g}}}^{1}\left(\pi^{*} \mathcal{I}^{\mathrm{g}}, \mathcal{L}_{\operatorname{og}^{\mathrm{g}}}^{\mathrm{g}}(1)\right)
$$

\subsection{The comparison of $\mathcal{P}$ ol and $\mathcal{P}_{\mathrm{ol}}^{\mathrm{g}}$}

In this section we compare $\mathcal{P} \mathrm{ol}$ and $\mathcal{P}_{\mathrm{ol}}{ }^{\mathrm{g}}$. Recall that $\mathcal{P}_{\mathrm{ol}}{ }^{\mathrm{g}}$ is only defined for $\Lambda=\mathbb{Z} / l^{r} \mathbb{Z}$.

\subsubsection{Comparison of the logarithm sheaves}

We first reformulate the property of being a continuous $\mathcal{R}^{\mathrm{g}}$-module on $S$. Choose a geometric point $\bar{s} \in S$ and define a ring

$$
\Lambda\left[\left[\mathcal{H}_{\left.\mathbb{Z}_{l}, \bar{s}\right]}\right]:=\varliminf_{n}\left(\mathcal{R}_{n}^{\mathrm{g}}\right)_{\bar{s}}=\varliminf_{n} \Lambda\left[H_{n, \bar{s}}\right] .\right.
$$

Then a lisse $\Lambda$-sheaf $\mathcal{M}$ on $S$ with an action of $\mathcal{R}_{n}^{\mathrm{g}}$ for some $n$ is the same as a finite $\Lambda$-module with a continuous action of $\Lambda\left[\left[\mathcal{H}_{\left.\mathbb{Z}_{l}, \bar{s}\right]}\right]\right.$. We call these continuous $\Lambda\left[\left[\mathcal{H}_{\mathbb{Z}_{l}, \bar{s}}\right]\right]$-modules. We define two ideals in $\Lambda\left[\left[\mathcal{H}_{\mathbb{Z}_{l}, \bar{s}}\right]\right.$ :

$$
\begin{aligned}
\mathfrak{J}_{n} & :=\operatorname{ker}\left(\Lambda\left[\left[\mathcal{H}_{\left.\mathbb{Z}_{l}, \bar{s}\right]}\right] \rightarrow \mathcal{R}_{n}^{\mathrm{g}}\right)\right. \\
\mathfrak{a} & :=\operatorname{ker}\left(\Lambda\left[\left[\mathcal{H}_{\mathbb{Z}_{l}, \bar{s}}\right]\right] \rightarrow \Lambda\right) .
\end{aligned}
$$

The universal property 3.3 .2 of $\mathcal{L}_{\mathrm{og}} \mathrm{g}$ and the section $1^{(k)}$ of $e^{*} \mathcal{L}_{\Lambda}^{(k)}$ implies:

Lemma 3.4.1. Let $\Lambda=\mathbb{Z} / l^{r} \mathbb{Z}$, then there is a unique map

$$
\varrho^{(k)} \in \underline{\lim }_{n} \bar{\pi}_{*} \underline{\operatorname{Hom}}_{E}\left(\mathcal{L}_{\operatorname{og}_{n}^{\mathrm{g}}}^{\mathrm{g}}, \mathcal{L}_{\mathrm{og}_{\Lambda}^{(k)}}\right)
$$

corresponding to $1^{(k)}: \Lambda \rightarrow e^{*} \mathcal{L}_{\mathrm{og}}^{(k)}$. 
In fact we want to show that $\varrho^{(k)}$ induces an isomorphism of pro-sheaves $\varrho:\left(\mathcal{L}_{\log }^{\mathrm{g}}\right)_{n} \rightarrow\left(\mathcal{L}_{\operatorname{og}_{\Lambda}^{(k)}}^{(k)}\right)_{k}$.

Lemma 3.4.2. Let $\Lambda=\mathbb{Z} / l^{r} \mathbb{Z}$ and let $\mathfrak{a}^{k}$ be the $k$-th power of $\mathfrak{a}$, then $\mathfrak{a}^{k} / \mathfrak{a}^{k+1} \cong$ $\operatorname{Sym}^{k}\left(\mathcal{H}_{\mathbb{Z}_{l}} \otimes \Lambda\right)$ and there is a canonical surjection

$$
\varrho_{r+k-2}^{(k)}: \mathcal{R}_{r+k-2, \bar{s}}^{\mathrm{g}}=\Lambda\left[\left[\mathcal{H}_{\mathbb{Z}_{l}, \bar{s}}\right]\right] / \mathfrak{J}_{r+k-2} \rightarrow \Lambda\left[\left[\mathcal{H}_{\mathbb{Z}_{l}, \bar{s}}\right]\right] / \mathfrak{a}^{k}
$$

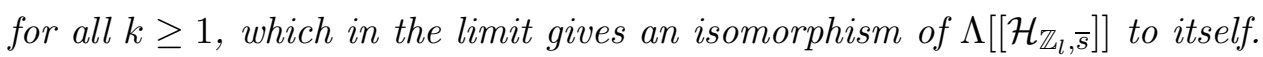

Proof. Let $\bar{s} \in S$ be a geometric point. Then we choose two topological generators $\gamma, \eta$ of $\mathcal{H}_{\mathbb{Z}_{l}, \bar{s}}$. It is a standard fact in Iwasawa theory that we have a ring isomorphism $\Lambda\left[\left[\mathcal{H}_{\left.\mathbb{Z}_{l}, \bar{s}\right]}\right] \cong \Lambda[[X, Y]]\right.$, mapping $\gamma-1 \mapsto X$ and $\eta-1 \mapsto Y$, with the power series ring in two variables. This implies that the augmentation ideal $\mathfrak{a}$ is mapped to the ideal $(X, Y)$. The claim that $\mathfrak{a}^{k} / \mathfrak{a}^{k+1} \cong \operatorname{Sym}^{k}\left(\mathcal{H}_{\mathbb{Z}_{l}} \otimes \Lambda\right)$ follows immediately. Now $\mathfrak{J}_{k}$ corresponds under this isomorphism to the ideal $\left((X+1)^{l^{k}}-1,(Y+1)^{l^{k}}-1\right)$. By induction one sees that this ideal is contained in $(l, X, Y)^{k+1}$. As $l^{r}=0$ in $\Lambda$ we have $(l, X, Y)^{r+k} \subset(X, Y)^{k+1}$. This gives the map as indicated, which in the limit is clearly an isomorphism.

Denote by $G^{(k)}$ the sheaf on $E$ defined by the $\mathcal{R}_{r+k-2}^{\mathrm{g}}$-module $\Lambda\left[\left[\mathcal{H}_{\mathbb{Z}_{l}, \bar{s}}\right]\right] / \mathfrak{a}^{k}$ via the equivalence of categories in 3.3.3. This is a unipotent sheaf with a section 1 of $e^{*} G^{(k)}$ given by the identity in $\Lambda\left[\left[\mathcal{H}_{\mathbb{Z}_{l}, \bar{s}}\right]\right] / \mathfrak{a}^{k}$. Thus, by 3.2 .2 there is a unique map $\mathcal{L}_{\mathrm{og}}{ }^{(k)} \rightarrow G^{(k)}$ mapping $1^{(k)}$ to 1 .

Proposition 3.4.3. Let $\Lambda=\mathbb{Z} / l^{r} \mathbb{Z}$. The map $\log ^{(k)} \rightarrow G^{(k)}$ is an isomorphism. In particular the map of pro-sheaves $\varrho: \mathcal{L}_{\mathrm{og}_{\Lambda}^{\mathrm{g}}} \rightarrow \mathcal{L}_{\mathrm{og}_{\Lambda}}$ defined above is an isomorphism.

Proof. The surjection $\mathcal{L}_{\mathrm{og}_{r+k-2}^{\mathrm{g}}} \rightarrow G^{(k)}$ factors through $\mathcal{L}_{\operatorname{og}}(k) \rightarrow G^{(k)}$ so that this map is also surjective. As these two sheaves are finite of the same cardinality, this is also an isomorphism.

\subsubsection{Comparison with the $\mathbb{Z}_{l}$-version}

Let $\Lambda_{r}:=\mathbb{Z} / l^{r} \mathbb{Z}$ and consider the pro-sheaf in $r$ and $n$

$$
\left(\mathcal{L}_{\Lambda_{\Lambda}, n}^{\mathrm{g}}\right)_{r, n}
$$

where $\mathcal{L}_{\operatorname{og}_{\Lambda_{r+1}, n}^{\mathrm{g}}}^{\mathrm{g}} \rightarrow \mathcal{L}_{\mathrm{og}_{\Lambda_{r}, n}^{\mathrm{g}}}^{\mathrm{g}}$ is induced by the reduction map $\Lambda_{r+1} \rightarrow \Lambda_{r}$. We observe:

Lemma 3.4.4. For every $k \geq 0$, the projective system $\left(\mathcal{L}_{\operatorname{og}_{\Lambda_{r}, r+k-2}^{\mathrm{g}}}\right)_{r}$ is cofinal in $\left(\mathcal{L}_{\operatorname{og}_{\Lambda_{r}, n}}^{\mathrm{g}}\right)_{r, n}$. 
The $\mathbb{Z}_{l}$-version of $\mathcal{L} \mathrm{og}$ is reconstructed from the $\Lambda_{r}$-version of $\mathcal{L}_{\mathrm{og}}{ }^{\mathrm{g}}$ as follows:

Proposition 3.4.5. The map $\varrho^{(k)}$ induces an morphism of pro-sheaves

$$
\left(\mathcal{L}_{\Lambda^{\prime}, r+k-2}^{\mathrm{g}}\right)_{r} \rightarrow\left(\mathcal{L}_{\Lambda^{\prime}}^{(k)}\right)_{r}=\mathcal{L}_{\Lambda_{r}}^{(k)}
$$

The induced morphism of pro-sheaves

$$
\left.\varrho:\left(\mathcal{L}_{\Lambda^{\prime}, r}^{\mathrm{g}}\right)_{r} \cong\left(\mathcal{L}_{\Lambda^{\prime}, r+k-2}^{\mathrm{g}}\right)_{r, k} \rightarrow\left(\mathcal{L}_{\Lambda^{\prime}}^{(k)}\right)_{\mathbb{Z}_{l}}\right)_{k}=\mathcal{L}_{\operatorname{og}_{\mathbb{Z}_{l}}}
$$

is an isomorphism.

Proof. From lemma 3.4 .2 and the universal property of $\mathcal{L}_{\mathrm{og}} \mathrm{g}$ we get the morphism of $\left(\mathcal{L}_{\mathrm{og}_{\Lambda_{r}, r+k-2}^{\mathrm{g}}}^{\mathrm{g}}\right)_{r}$ to $\mathcal{L}_{\mathrm{og}_{\mathbb{Z}_{l}}}^{(k)}$. To check that this induces an isomorphism if take pro-sheaves in the index $k$, it is enough to check this after pull-back with $e^{*}$. We get the map

$$
\varliminf_{k} \lim _{r} \Lambda_{r}\left[H_{r+k-2, \bar{s}}\right] \rightarrow \lim _{k} \mathbb{Z}_{l}\left[\left[\mathcal{H}_{\left.\mathbb{Z}_{l}, \bar{s}\right]}\right]\right] / \mathfrak{a}^{k},
$$

which is an isomorphism.

\subsubsection{Comparison of polylogarithm sheaves}

Using the functor $H_{e}$ from 3.2.7 we can translate the comparison results for the logarithm sheaves to the polylog.

Proposition 3.4.6. The isomorphism @ form 3.4.5 induces an isomorphism of pro-sheaves

$$
H_{e}(\varrho):\left(\mathcal{P O l}_{\Lambda_{r}, r}^{\mathrm{g}}\right)_{r}=\left(H_{e}\left(\mathcal{L}_{\mathrm{og}_{\Lambda_{r}, r}^{\mathrm{g}}}^{\mathrm{g}}(1)\right)\right)_{r} \stackrel{\cong}{\rightarrow} \mathcal{P}_{\mathrm{ol}_{\mathbb{Z}_{l}}} .
$$

Proof. Clear from the definition.

\subsection{Specialization of the elliptic polylogarithm sheaf, $l$-adic Eisenstein classes}

The specialization along torsion sections of the elliptic polylog gives interesting cohomology classes. These are the $l$-adic Eisenstein classes investigated in Hu-Ki1, Hu-Ki2]. We recall their construction. 


\subsubsection{Invariance of the logarithm sheaf under translation by torsion sections}

Let $N \in \mathbb{Z}$ be invertible on $S$ and $[N]: E \rightarrow E$ the $N$-multiplication. The universal property 3.2 .2 gives us canonical maps sending $1^{(k)}$ to $[N]^{*} 1^{(k)}$

$$
\mathcal{L}_{\Lambda}^{(k)} \rightarrow[N]^{*} \mathcal{L}_{\Lambda}^{(k)}
$$

for $\Lambda=\mathbb{Z} / l^{r} \mathbb{Z}, \mathbb{Z}_{l}$ or $\mathbb{Q}_{l}$. Thus for every $N$-torsion point $t: S \rightarrow E$ we get a map of pro-sheaves

$$
\operatorname{pr}_{t}^{N}: t^{*} \mathcal{L}_{\operatorname{og}} \rightarrow e^{*} \mathcal{L}_{\operatorname{og}_{\Lambda}}
$$

Similarly, we have a map

$$
\operatorname{pr}_{t}^{N}: \mathcal{L}_{\operatorname{og}_{n}^{\mathrm{g}}}^{\mathrm{g}} \rightarrow[N]^{*} \mathcal{L}_{\operatorname{og}_{n}^{\mathrm{g}}}^{\mathrm{g}}
$$

for the geometric logarithm sheaf.

Lemma 3.5.1. If $l \nmid N$, then the map $\mathrm{pr}_{t}^{N}$ is an isomorphism.

Proof. Let us first treat the unipotent case. It suffices to show that $\log _{\Lambda} \rightarrow$ $[N]^{*} \mathcal{L}_{\operatorname{og}_{\Lambda}}$ is an isomorphism. Using the equivalence of categories 3.2.4, this can be tested after pull-back with $e^{*}$. The resulting map $\mathcal{R}_{\Lambda} \rightarrow \mathcal{R}_{\Lambda}$ is on the the $k$-th graded piece $\operatorname{Sym}^{k} \mathcal{H}_{\Lambda}$ induced by the map $\mathcal{H}_{\Lambda} \stackrel{[N]^{*}}{\longrightarrow} \mathcal{H}_{\Lambda}$, which is just the $N$-multiplication and thus an isomorphism. In the geometric case we have $t^{*} \mathcal{L}_{\mathrm{og}_{n}^{\mathrm{g}}}^{\mathrm{g}}=\Lambda\left[p_{n}^{-1}(t)\right]$ and $e^{*} \mathcal{L}_{\mathrm{og}_{n}^{\mathrm{g}}}^{\mathrm{g}}=\Lambda\left[H_{n}\right]$. The map $\operatorname{pr}_{t}^{N}$ is by definition induced by $t_{n} \mapsto[N] t_{n}$ for $t_{n} \in p_{n}^{-1}(t)$. This is obviously an isomorphism if $l \nmid N$.

To define a morphism $\mathrm{pr}_{t}$ independent of $N$, we let

$$
\operatorname{pr}_{t}:=\operatorname{pr}_{t}^{N} \circ\left(\operatorname{pr}_{e}^{N}\right)^{-1}: t^{*} \mathcal{L}_{\mathrm{og}_{\Lambda}} \rightarrow e^{*} \mathcal{L}_{\mathrm{og}_{\Lambda}}
$$

We need an explicit description of the map $\mathrm{pr}_{t}$ on the geometric logarithm sheaf.

We have $t^{*} \mathcal{L}_{\operatorname{og}_{n}^{\mathrm{g}}}^{\mathrm{g}}=\Lambda\left[p_{n}^{-1}(t)\right]$ and $e^{*} \mathcal{L}_{\operatorname{og}_{n}^{\mathrm{g}}}=\Lambda\left[H_{n}\right]$. Here $p_{n}^{-1}(t) \subset E\left[N l^{n}\right]$ and if $l \nmid N$ we have $E\left[N l^{n}\right]=E[N] \oplus E\left[l^{n}\right]$.

Lemma 3.5.2. The map from (8)

$$
\operatorname{pr}_{t}: \Lambda\left[p_{n}^{-1}(t)\right] \rightarrow \Lambda\left[H_{n}\right]
$$

is induced by the projection of $t_{n} \in p_{n}^{-1}(t) \subset E[N] \oplus E\left[l^{n}\right]$ to $H_{n}=E\left[l^{n}\right]$. 
Proof. The map $\operatorname{pr}_{t}^{N}: \Lambda\left[p_{n}^{-1}(t)\right] \rightarrow \Lambda\left[H_{n}\right]$ maps $t_{n} \in p_{n}^{-1}(t)$ to $[N] t_{n} \in H_{n}$. This gives the result.

Passing to the limit we get a map of pro-sheaves

$$
\operatorname{pr}_{t}: \mathbb{Z}_{l}\left[\left[\mathcal{H}_{\mathbb{Z}_{l}, t}\right]\right] \rightarrow \mathbb{Z}_{l}\left[\left[\mathcal{H}_{\mathbb{Z}_{l}}\right]\right]
$$

where $\mathcal{H}_{\mathbb{Z}_{l}, t}:=\varliminf_{n} p_{n}^{-1}(t)$. This is induced by the projection $\mathcal{H}_{\mathbb{Z}_{l}, t} \rightarrow \mathcal{H}_{\mathbb{Z}_{l}}$.

\subsubsection{The moment map}

The pro-sheaf $e^{*} \log _{\mathbb{Q}_{l}}=\mathcal{R}_{\mathbb{Q}_{l}}$ is a Hopf algebra (see Be-Le 1.2.10 iv)) and

the map $\nu: \operatorname{Sym}^{\leq k} \mathcal{H}_{\mathbb{Q}_{l}} \rightarrow \mathrm{Gr}^{\leq k} \mathcal{R}_{\mathbb{Q}_{l}}^{(k)}$ is an isomorphism. Let $\hat{\mathfrak{U}}\left(\mathcal{H}_{\mathbb{Q}_{l}}\right)$ be the completion of the universal enveloping algebra of the abelian Lie algebra $\mathcal{H}_{\mathbb{Q}_{l}}$. The canonical filtration makes this a pro-sheaf $\left(\mathfrak{U}^{k}\left(\mathcal{H}_{\mathbb{Q}_{l}}\right)\right)_{k}$.

The structure theorem, Bour ch. II, paragraph 1, no. 6, gives:

Lemma 3.5.3. The map $\nu: \mathcal{H}_{\mathbb{Q}_{l}} \rightarrow \mathcal{R}_{\mathbb{Q}_{l}}$ extends to an isomorphism of Hopf algebra pro-sheaves

$$
\nu: \hat{\mathfrak{U}}\left(\mathcal{H}_{\mathbb{Q}_{l}}\right) \cong \mathcal{R}_{\mathbb{Q}_{l}}
$$

which on the $k$-th graded piece $\operatorname{Sym}^{k} \mathcal{H}_{\mathbb{Q}_{l}}$ is multiplication by $k$ !

Recall from 3.4.5 that we have an isomorphism

$$
\varrho:\left(\mathcal{R}_{\Lambda_{r}}^{\mathrm{g}}\right)_{r} \cong \mathcal{R}_{\mathbb{Z}_{l}} .
$$

Definition 3.5.4. We define the $k$-th moment map $\mu^{k}$ to be the composition

$$
\mu^{k}:\left(\mathcal{R}_{\Lambda_{r}}^{\mathrm{g}}\right)_{r} \cong \mathcal{R}_{\mathbb{Z}_{l}} \rightarrow \mathcal{R}_{\mathbb{Q}_{l}} \cong \hat{\mathfrak{U}}\left(\mathcal{H}_{\mathbb{Q}_{l}}\right) \rightarrow \operatorname{Sym}^{k} \mathcal{H}_{\mathbb{Q}_{l}}
$$

where the last map is the projection onto the $k$-th factor.

We need an explicit description of the moment map. For this we write $\left(\mathcal{R}_{n+k-2, \Lambda_{n}}^{\mathrm{g}}\right)_{n}=\left(\mathcal{R}_{\Lambda_{r}}^{\mathrm{g}}\right)_{r}$ and we describe $\mu^{k}$ on $\Lambda_{n}\left[H_{n+k-2}\right]=\mathcal{R}_{n+k-2, \Lambda_{n}}^{\mathrm{g}}$.

Lemma 3.5.5. Write $\operatorname{Sym}^{k} \mathcal{H}_{\mathbb{Q}_{l}}=\operatorname{Sym}^{k} \mathcal{H}_{\mathbb{Z}_{l}} \otimes \mathbb{Q}_{l}$, then

$$
\begin{aligned}
\mu^{k}:\left(\Lambda_{n}\left[H_{n+k-2}\right]\right)_{n} & \rightarrow\left(\operatorname{Sym}^{k} H_{n}\right)_{n} \otimes \mathbb{Q}_{l} \\
\left(\sum_{h \in H_{n+k-2}} n_{h}(h)\right)_{n} & \mapsto\left(\sum_{h \in H_{n}} n_{h}\left(h^{\otimes k}\right)\right)_{n} \otimes \frac{1}{k !} .
\end{aligned}
$$

Proof. This follows immediately from the definition of the maps $\mu^{k}$ and $\varrho$. 


\subsubsection{A splitting}

Lemma 3.5.6. Let $\Lambda=\mathbb{Z} / l^{r} \mathbb{Z}, \mathbb{Z}_{l}$ or $\mathbb{Q}_{l}$. Then the inclusion $\mathcal{I}_{\Lambda} \hookrightarrow \mathcal{R}_{\Lambda}$ induces an injective map

$$
\mathcal{R}_{\Lambda}(1)=\underline{\operatorname{Hom}}_{S, \mathcal{R}_{\Lambda}}\left(\mathcal{R}_{\Lambda}, \mathcal{R}_{\Lambda}(1)\right) \rightarrow \underline{\operatorname{Hom}}_{S, \mathcal{R}_{\Lambda}}\left(\mathcal{I}_{\Lambda}, \mathcal{R}_{\Lambda}(1)\right),
$$

which is an isomorphism for $\Lambda=\mathbb{Q}_{l}$.

Proof. This follows from $\underline{\operatorname{Hom}}_{S, \mathcal{R}_{\Lambda}}\left(\Lambda, \mathcal{R}_{\Lambda}(1)\right)=0$ and $\underline{\operatorname{Ext}}_{S, \mathcal{R}_{\mathbb{Q}_{l}}}^{1}\left(\mathbb{Q}_{l}, \mathcal{R}_{\mathbb{Q}_{l}}(1)\right)=$ 0 , which is a consequence of the Kozsul resolution as $\mathcal{R}_{\mathbb{Q}_{l}}$ is a sheaf of regular rings.

Corollary 3.5.7. The local to global spectral sequence for Ext gives an injection

$\operatorname{Ext}_{S, \mathbb{Q}_{l}}^{1}\left(\mathbb{Q}_{l}, \mathcal{R}_{\mathbb{Q}_{l}}(1)\right) \cong \operatorname{Ext}_{S, \mathcal{R}_{\mathbb{Q}_{l}}}^{1}\left(\mathcal{R}_{\mathbb{Q}_{l}}, \underline{\operatorname{Hom}}_{S, \mathcal{R}_{\mathbb{Q}_{l}}}\left(\mathcal{I}_{\mathbb{Q}_{l}}, \mathcal{R}_{\mathbb{Q}_{l}}(1)\right)\right) \stackrel{a}{\rightarrow} \operatorname{Ext}_{S, \mathcal{R}_{\mathbb{Q}_{l}}}^{1}\left(\mathcal{I}_{\mathbb{Q}_{l}}, \mathcal{R}_{\mathbb{Q}_{l}}(1)\right)$

Using the isomorphism $\nu: \mathcal{R}_{\mathbb{Q}_{l}} \cong \hat{\mathfrak{U}}\left(\mathcal{H}_{\mathbb{Q}_{l}}\right)$ we have an exact sequence (Kozsul resolution for the Lie algebra $\left.\mathcal{H}_{\mathbb{Q}_{l}}\right)$

$$
0 \rightarrow \mathcal{R}_{\mathbb{Q}_{l}}(1) \rightarrow \mathcal{H}_{\mathbb{Q}_{l}} \otimes_{\mathbb{Q}_{l}} \mathcal{R}_{\mathbb{Q}_{l}} \stackrel{b}{\rightarrow} \mathcal{I}_{\mathbb{Q}_{l}} \rightarrow 0
$$

Here we used $\Lambda^{2} \mathcal{H}_{\mathbb{Q}_{l}} \cong \mathbb{Q}_{l}(1)$ induced by the Weil-pairing. This map $b$ induces

$$
\begin{aligned}
& \operatorname{Ext}_{S, \mathbb{Q}_{l}}^{1}\left(\mathbb{Q}_{l}, \mathcal{R}_{\mathbb{Q}_{l}}(1)\right) \stackrel{a}{\longrightarrow} \quad \operatorname{Ext}_{S, \mathcal{R}_{\mathbb{Q}_{l}}}^{1}\left(\mathcal{I}_{\mathbb{Q}_{l}}, \mathcal{R}_{\mathbb{Q}_{l}}(1)\right) \\
& \downarrow b^{*} \\
& \operatorname{Ext}_{S, \mathcal{R}_{\mathbb{Q}_{l}}}^{1}\left(\mathcal{H}_{\mathbb{Q}_{l}} \otimes_{\mathbb{Q}_{l}} \mathcal{R}_{\mathbb{Q}_{l}}, \mathcal{R}_{\mathbb{Q}_{l}}(1)\right) \\
& \downarrow= \\
& \operatorname{Ext}_{S, \mathbb{Q}_{l}}^{1}\left(\mathcal{H}_{\mathbb{Q}_{l}}, \mathcal{R}_{\mathbb{Q}_{l}}(1)\right) \text {. }
\end{aligned}
$$

Lemma 3.5.8. The map

$$
\operatorname{Ext}_{S, \mathbb{Q}_{l}}^{1}\left(\mathbb{Q}_{l}, \mathcal{R}_{\mathbb{Q}_{l}}(1)\right) \stackrel{b^{*} \circ a}{\longrightarrow} \operatorname{Ext}_{S, \mathbb{Q}_{l}}^{1}\left(\mathcal{H}_{\mathbb{Q}_{l}}, \mathcal{R}_{\mathbb{Q}_{l}}(1)\right)
$$

has a canonical splitting. 
Proof. We have an isomorphism

$$
\operatorname{Ext}_{S, \mathbb{Q}_{l}}^{1}\left(\mathcal{H}_{\mathbb{Q}_{l}}, \mathcal{R}_{\mathbb{Q}_{l}}(1)\right) \cong \operatorname{Ext}_{S, \mathbb{Q}_{l}}^{1}\left(\mathbb{Q}_{l}, \underline{\operatorname{Hom}}_{S, \mathbb{Q}_{l}}\left(\mathcal{H}_{\mathbb{Q}_{l}}, \mathcal{R}_{\mathbb{Q}_{l}}(1)\right)\right)
$$

and a contraction map

$$
\left.\underline{\operatorname{Hom}}_{S, \mathbb{Q}_{l}}\left(\mathcal{H}_{\mathbb{Q}_{l}}, \mathcal{R}_{\mathbb{Q}_{l}}(1)\right)\right) \rightarrow \mathcal{R}_{\mathbb{Q}_{l}}(1)
$$

given on

$$
\underline{\operatorname{Hom}}_{S, \mathbb{Q}_{l}}\left(\mathcal{H}_{\mathbb{Q}_{l}}, \operatorname{Sym}^{k} \mathcal{H}_{\mathbb{Q}_{l}}(1)\right) \cong \underline{\operatorname{Hom}}_{S, \mathbb{Q}_{l}}\left(\mathcal{H}_{\mathbb{Q}_{l}}, \mathbb{Q}_{l}\right) \otimes \operatorname{Sym}^{k} \mathcal{H}_{\mathbb{Q}_{l}}(1)
$$

by

$$
\begin{aligned}
& \underline{\operatorname{Hom}}_{S, \mathbb{Q}_{l}}\left(\mathcal{H}_{\mathbb{Q}_{l}}, \mathbb{Q}_{l}\right) \otimes \operatorname{Sym}^{k} \mathcal{H}_{\mathbb{Q}_{l}}(1) \rightarrow \operatorname{Sym}^{k-1} \mathcal{H}_{\mathbb{Q}_{l}}(1) \\
& f \otimes h_{1} \otimes \ldots \otimes h_{k} \mapsto \frac{1}{k+1} \sum_{i=1}^{k} f\left(h_{i}\right) h_{1} \otimes \ldots \hat{h}_{i} \ldots \otimes h_{k} .
\end{aligned}
$$

This gives the required map and it is straightforward to check that this is indeed a splitting of $b^{*} \circ a$.

\subsubsection{The specialization of the polylogarithm, $l$-adic Eisenstein classes}

We now define the specialization of the elliptic polylogarithm. Let $\beta \in$ $\mathbb{Z}[E[N](S) \backslash e]$ be of the form

$$
\beta=\sum_{t \in E[N](S) \backslash e} n_{t} t
$$

We want to define an element

$$
\left(\beta^{*} \mathcal{P}_{\mathrm{ol}_{\mathbb{Q}_{l}}}\right)^{k} \in H^{1}\left(S, \operatorname{Sym}^{k} \mathcal{H}_{\mathbb{Q}_{l}}(1)\right) .
$$

First observe that $\operatorname{pr}_{t} t^{*} \mathcal{P}_{\mathrm{Ol}_{\mathbb{Q}_{l}}}$ is an element in

$\operatorname{Ext}_{S, \mathcal{R}_{\mathbb{Q}_{l}}}^{1}\left(\mathcal{I}_{\mathbb{Q}_{l}}, t^{*} \mathcal{L}_{\operatorname{og}_{\mathbb{Q}_{l}}}(1)\right) \stackrel{\operatorname{pr}_{t}}{\longrightarrow} \operatorname{Ext}_{S, \mathcal{R}_{\mathbb{Q}_{l}}}^{1}\left(\mathcal{I}_{\mathbb{Q}_{l}}, e^{*} \mathcal{L}_{\operatorname{og}_{\mathbb{Q}_{l}}}(1)\right)=\operatorname{Ext}_{S, \mathcal{R}_{\mathbb{Q}_{l}}}^{1}\left(\mathcal{I}_{\mathbb{Q}_{l}}, \mathcal{R}_{\mathbb{Q}_{l}}(1)\right)$.

Define

$$
\sigma: \operatorname{Ext}_{S, \mathcal{R}_{\mathbb{Q}_{l}}}^{1}\left(\mathcal{I}_{\mathbb{Q}_{l}}, \mathcal{R}_{\mathbb{Q}_{l}}(1)\right) \rightarrow \operatorname{Ext}_{S, \mathbb{Q}_{l}}^{1}\left(\mathbb{Q}_{l}, \mathcal{R}_{\mathbb{Q}_{l}}(1)\right)
$$

to be the composition of $b^{*}$ and the splitting of lemma 3.5.8. This is a splitting of $a$. Denote by $\sigma^{k}$ the projection onto

$$
\operatorname{Ext}_{S, \mathbb{Q}_{l}}^{1}\left(\mathbb{Q}_{l}, \operatorname{Sym}^{k} \mathcal{H}_{\mathbb{Q}_{l}}(1)\right)=H^{1}\left(S, \operatorname{Sym}^{k} \mathcal{H}_{\mathbb{Q}_{l}}(1)\right) .
$$


Definition 3.5.9. For $\beta=\sum_{t \in E[N](S) \backslash e} n_{t} t \in \mathbb{Z}[E[N](S) \backslash e]$ define

$$
\left(\beta^{*} \mathcal{P}_{\mathrm{ol}_{\mathbb{Q}_{l}}}\right)^{k}:=\sum_{t \in E[N](S) \backslash e} n_{t}\left(\sigma^{k} \operatorname{pr}_{t} t^{*} \mathcal{P}_{\mathrm{ol}_{\mathbb{Q}_{l}}}\right) \in H^{1}\left(S, \operatorname{Sym}^{k} \mathcal{H}_{\mathbb{Q}_{l}}(1)\right)
$$

These are the $l$-adic Eisenstein classes associated to $\beta$.

Remark: This numbering disagrees with the one of [Hu-Ki2], where we wrote $\left(\beta^{*} \mathcal{P}_{\mathrm{ol}_{\mathbb{Q}_{l}}}\right)^{k+1}$ for this class.

Our aim is to compute not the specialization but a variant of it. Namely let $[\mathfrak{a}]: E \rightarrow E$ be an isogeny of degree $\mathrm{Na}:=\operatorname{deg}[\mathfrak{a}]$ prime to $l$ (the notations are chosen to fit the $\mathrm{CM}$ case which is our ultimate goal). Consider the operator $\left([\mathfrak{a}]^{*}-\mathrm{N} \mathfrak{a}\right)$ on $H^{1}\left(S, \operatorname{Sym}^{k} \mathcal{H}_{\mathbb{Q}_{l}}(1)\right)$. Here $[\mathfrak{a}]^{*}$ is the pull-back with $[\mathfrak{a}]$ and $\mathrm{Na}$ is the multiplication by the number $\operatorname{deg}[\mathfrak{a}] \in \mathbb{Z}$. This acts on $H^{1}\left(S, \operatorname{Sym}^{k} \mathcal{H}_{\mathbb{Q}_{l}}(1)\right)$ as $\mathfrak{a}^{k} \mathrm{Na}-\mathrm{N} \mathfrak{a}$, which is not zero if $k \neq 0$. Thus for $k \neq 0$ it is the same to compute

$$
\left([\mathfrak{a}]^{*}-\mathrm{Na}\right)\left(\beta^{*} \mathcal{P}_{\mathrm{ol}_{\mathbb{Q}_{l}}}\right)^{k} \in H^{1}\left(S, \operatorname{Sym}^{k} \mathcal{H}_{\mathbb{Q}_{l}}(1)\right)
$$

or $\left(\beta^{*} \mathcal{P}_{\mathrm{ol}_{\mathbb{Q}_{l}}}\right)^{k}$.

\section{The l-adic realization of the elliptic polylog}

This part is concerned with the construction of the polylog on elliptic curves in a geometric way, which allows to compute its specializations explicitly. This is the technical heart of the paper and in our opinion our main contribution to the problem of the Tamagawa number conjecture for elliptic curves.

\subsection{The polylog as a one-motive}

From the definition of the polylogarithm it is quite obvious that it is a prosheaf consisting of $\mathbb{Z} / l^{r} \mathbb{Z}$-realizations of one-motives. In this section we make this connection more explicit.

\subsubsection{A reformulation}

Let $\Lambda_{r}=\mathbb{Z} / l^{r} \mathbb{Z}$ and recall from (5) that the geometric polylog sits in an exact sequence

$$
0 \rightarrow \pi^{*} \bar{\pi}_{*} \mathcal{L}_{\operatorname{og}_{n}^{\mathrm{g}}}^{\mathrm{g}}(1) \rightarrow \mathcal{L}_{\operatorname{og}_{n}^{\mathrm{g}}}^{\mathrm{g}}(1)_{U} \rightarrow \mathcal{P}_{\mathrm{ol}}^{\mathrm{g}} \rightarrow \pi^{*} R^{1} \pi_{*} \mathcal{L}_{\operatorname{og}_{n, U}^{\mathrm{g}}}^{\mathrm{g}}(1) \rightarrow 0
$$


By the definition of the geometric logarithm $\mathcal{L}_{0_{n}^{g}}^{\mathrm{g}}=p_{n *} \Lambda_{r}$ and hence $\bar{\pi}_{*} \mathcal{L}_{\mathrm{og}_{n}^{\mathrm{g}}}^{\mathrm{g}}=$ $\Lambda_{r}$. We get

$$
0 \rightarrow \Lambda_{r}(1) \rightarrow p_{n *} \Lambda_{r, U}(1) \rightarrow \mathcal{P}_{\mathrm{ol}}^{\mathrm{g}} \rightarrow \pi^{*} R^{1} \pi_{n *} \Lambda_{r, U}(1) \rightarrow 0
$$

For a lisse sheaf $\mathcal{F}$ on $U$ consider the dual

$$
(\mathcal{F})^{\vee}:=\underline{\operatorname{Hom}}_{U}(\mathcal{F}, \Lambda)
$$

Then, using Poincaré duality, we get by dualizing and twisting by 1 an exact sequence

$$
0 \rightarrow \pi_{n}^{*} R^{1} \pi_{n !} \Lambda_{r, U}(1) \rightarrow\left(\mathcal{P}_{\mathrm{ol}}^{\mathrm{g}}\right)^{\vee}(1) \rightarrow p_{n *} \Lambda_{r, U} \rightarrow \Lambda_{r, U} \rightarrow 0 .
$$

Denote by $I_{n, \Lambda_{r}}$ the kernel of the map $p_{n *} \Lambda_{r, U} \rightarrow \Lambda_{r, U}$, then $\left(\mathcal{P}_{\mathrm{ol}}^{\mathrm{g}}\right)^{\vee}(1)$ gives a class in

$$
\operatorname{Ext}_{U}^{1}\left(I_{n, \Lambda_{r}}, \pi_{n}^{*} R^{1} \pi_{n !} \Lambda_{r, U}(1)\right)
$$

We want to give a geometric interpretation of this class. For this we will relate $\left(\mathcal{P}_{0} l_{n}^{\mathrm{g}}\right)^{\vee}(1)$ with the $l^{r}$-torsion points of a one-motive, which is defined via a generalized Picard scheme.

\subsubsection{The generalized Picard scheme}

We will give a geometric interpretation of $\left(\mathcal{P}_{\mathrm{ol}}^{\mathrm{g}}\right)^{\vee}(1)$. For this we need the Picard scheme of line bundles on $E_{n}$ trivialized along $H_{n}$ (cf. the article by Raynaud [Ra]).

Definition 4.1.1. Let $P_{H_{n}}$ be the generalized Picard scheme representing the functor, which associates to $S^{\prime} \rightarrow S$ the isomorphism classes of pairs $(\mathcal{L}, \alpha)$, where $\mathcal{L}$ is a line bundle on $E_{n} \times_{S} S^{\prime}$ and $\alpha: h_{n}^{*} \mathcal{L} \cong \mathcal{O}_{H_{n}}$ is a trivialization of $\mathcal{L}$ along $H_{n} \times{ }_{S} S^{\prime}$.

That $H_{n}$ is a rigidificator in the sense of [Ra] follows from the fact that $H_{n}$ contains the section $e_{n}: S \rightarrow E_{n}$. Denote by $P_{n}$ the Picard scheme of $E_{n}$. Then we have an exact sequence

$$
0 \rightarrow T_{H_{n}} \rightarrow P_{H_{n}} \rightarrow P_{n} \rightarrow 0,
$$

where $T_{H_{n}}$ is the torus with character group $I\left[H_{n}\right]:=\operatorname{ker}\left(p_{n *} \mathbb{Z} \rightarrow \mathbb{Z}\right)$. The $l^{r}$-torsion of $P_{H_{n}}$ can be identified as follows: 
Lemma 4.1.2. There is a canonical isomorphism

$$
R^{1} \pi_{n !} \Lambda_{r}(1) \cong P_{H_{n}}\left[l^{r}\right]
$$

Proof. Define $H_{n} \mathbb{G}_{m}:=\operatorname{ker}\left(\mathbb{G}_{m} \rightarrow h_{n *} h_{n}^{*} \mathbb{G}_{m}\right)$. Then $P_{H_{n}}=R^{1} \bar{\pi}_{*}\left(H_{n} \mathbb{G}_{m}\right)$ (see [SGA4,III, Expose XVIII proposition 1.5.14), where the higher direct image is taken for the flat topology. The sequence from loc. cit. lemma 1.6.1

$$
0 \rightarrow j_{n !} \mu_{l^{r}} \rightarrow_{H_{n}} \mathbb{G}_{m} \stackrel{\left[l^{r}\right]}{\longrightarrow} H_{n} \mathbb{G}_{m} \rightarrow 0
$$

gives an isomorphism $R^{1} \bar{\pi}_{*} j_{n !} \mu_{l^{r}} \cong P_{H_{n}}\left[l^{r}\right]$.

On $E_{n} \times{ }_{S} U_{n}$ we have the line bundle $\mathcal{O}\left(\Delta_{n}\right)$ associated to the Cartier divisor defined by the diagonal $\Delta_{n}$. By definition $\mathcal{O}\left(\Delta_{n}\right)$ sits in an exact sequence

$$
0 \rightarrow \mathcal{O} \rightarrow \mathcal{O}\left(\Delta_{n}\right) \rightarrow \Delta_{n *} \mathcal{O}_{U_{n}} \rightarrow 0
$$

which induces a trivialization of $\mathcal{O}\left(\Delta_{n}\right)$ along $H_{n} \times{ }_{S} U_{n}$. Thus we get a section $\Delta_{n}: \mathbb{Z} \rightarrow \pi_{n}^{*} P_{H_{n}}$ of étale sheaves. Adjunction gives a map

$$
\Delta_{n}: p_{n *} \mathbb{Z} \rightarrow \pi^{*} P_{H_{n}}
$$

also denoted by $\Delta_{n}$ by abuse of notation.

\subsubsection{Comparison with a one-motive}

Consider $p_{n *} \mathbb{Z} \rightarrow \pi^{*} P_{H_{n}}$ as a complex of sheaves in degree 0 and 1 . Note that $p_{n *} \mathbb{Z} \rightarrow \pi^{*} P_{H_{n}}$ is not a one-motive because $P_{H_{n}}$ is not a semi-abelian scheme. We have an exact sequence $0 \rightarrow P_{H_{n}}^{0} \rightarrow P_{H_{n}} \rightarrow \mathbb{Z} \rightarrow 0$ and if we let $I\left[H_{n}\right]$ be the kernel of the composition $p_{n *} \mathbb{Z} \rightarrow \mathbb{Z}$ we get a quasi-isomorphism

$$
\left[I\left[H_{n}\right] \rightarrow P_{H_{n}}^{0}\right] \cong\left[p_{n *} \mathbb{Z} \rightarrow \pi^{*} P_{H_{n}}\right]
$$

Here $I\left[H_{n}\right] \rightarrow P_{H_{n}}^{0}$ is of course a one-motive.

Theorem 4.1.3. There is a canonical isomorphism of étale sheaves

$$
\underline{H}^{0}\left(\left[p_{n *} \mathbb{Z} \rightarrow \pi^{*} P_{H_{n}}\right] \otimes^{\mathbb{L}} \mathbb{Z} / l^{r} \mathbb{Z}\right) \cong\left(\mathcal{P}_{\mathrm{ol}}^{\mathrm{g}}\right)^{\vee}(1)
$$

which is compatible with the morphism $\left(\mathcal{P}_{\mathrm{ol}}^{\mathrm{g}}\right)^{\vee}(1) \rightarrow\left(\mathcal{P}_{\mathrm{ol}}^{\mathrm{g}}\right)^{\vee}(1)$ induced by the trace map $\mathcal{L}_{\operatorname{og}_{m}^{\mathrm{g}}}^{\mathrm{g}} \rightarrow \mathcal{L}_{\mathrm{og}_{n}^{\mathrm{g}}}^{\mathrm{g}}$. 
Proof. Let $t: S \rightarrow E$ be a section and $H_{t, n}:=p_{n}^{-1}(t)$ be its preimage in $E_{n}$. Let $\varrho_{n}: H_{t, n} \rightarrow E_{n}$ be the embedding and denote by $g_{n}$ the open immersion of the complement

$$
g_{n}: E_{n} \backslash H_{t, n} \hookrightarrow E_{n} .
$$

Denote also by $p_{t, n}: H_{t, n} \rightarrow S$ the structure map. We have an exact sequence of étale sheaves

$$
0 \rightarrow \mathbb{G}_{m} \rightarrow g_{n *} g_{n}^{*} \mathbb{G}_{m} \rightarrow \varrho_{n *} \mathbb{Z} \rightarrow 0 .
$$

On the other hand let $H_{n} \mathbb{G}_{m}:=\operatorname{ker}\left(\mathbb{G}_{m} \rightarrow h_{n *} h_{n}^{*} \mathbb{G}_{m}\right)$, where $h_{n}: H_{n} \rightarrow E_{n}$ is the embedding. Then we get an exact sequence

$$
0 \rightarrow_{H_{n}} \mathbb{G}_{m} \rightarrow g_{n *} g_{n}^{*}\left(H_{n} \mathbb{G}_{m}\right) \rightarrow \varrho_{n *} \mathbb{Z} \rightarrow 0 .
$$

The long exact cohomology sequence for $R \bar{\pi}_{n *}$ taken for the flat topology gives

$$
\left(p_{t, n}\right)_{*} \mathbb{Z} \rightarrow \pi^{*} R^{1} \bar{\pi}_{n *}\left(H_{n} \mathbb{G}_{m}\right) \rightarrow R^{1} \bar{\pi}_{n *} g_{n *} g_{n}^{*}\left(H_{n} \mathbb{G}_{m}\right) \rightarrow 0
$$

because $R^{1} p_{t, n} \mathbb{Z}=0$. We have an identification $R^{1} \bar{\pi}_{n *}\left(H_{n} \mathbb{G}_{m}\right) \cong P_{H_{n}}$ and it is clear that the map $\left(p_{t, n}\right)_{*} \mathbb{Z} \rightarrow R^{1} \bar{\pi}_{n *}\left(H_{n} \mathbb{G}_{m}\right) \cong P_{H_{n}}$ is injective. We get an isomorphism of complexes

$$
\left[\left(p_{t, n}\right)_{*} \mathbb{Z} \rightarrow P_{H_{n}}\right] \otimes^{\mathbb{L}} \mathbb{Z} / l^{r} \mathbb{Z} \cong\left[R^{1} \bar{\pi}_{n *} g_{n *} g_{n}^{*}\left(H_{n} \mathbb{G}_{m}\right)[1]\right] \otimes^{\mathbb{L}} \mathbb{Z} / l^{r} \mathbb{Z} .
$$

Using the sequence

$$
0 \rightarrow j_{n !} \mu_{l^{r}} \rightarrow_{H_{n}} \mathbb{G}_{m} \stackrel{\left[l^{r}\right]}{\longrightarrow} H_{n} \mathbb{G}_{m} \rightarrow 0
$$

as in the proof of lemma 4.1.2, we see that

$$
\underline{H}^{0}\left(\left[\left(p_{t, n}\right)_{*} \mathbb{Z} \rightarrow \pi^{*} P_{H_{n}}\right] \otimes^{\mathbb{L}} \mathbb{Z} / l^{r} \mathbb{Z}\right) \cong R^{1} \bar{\pi}_{n *} g_{n *} g_{n}^{*}\left(j_{n !} \mu_{l^{r}}\right),
$$

where $j_{n}: U_{n} \rightarrow E_{n}$. Let us compute $t^{*} \mathcal{P}_{\mathrm{ol}}^{\mathrm{g}}$. By base change and the definition 3.2 .9 it is a straightforward computation that

$$
t^{*} \mathcal{P}_{\mathrm{ol}}^{\mathrm{g}}=R^{1} \bar{\pi}_{n *} g_{n !} g_{n}^{*} j_{n *} \mu_{l^{r}}
$$

and by Poincaré duality we get

$$
t^{*}\left(\mathcal{P}_{\mathrm{O}}^{\mathrm{g}}\right)^{\vee}(1)=R^{1} \bar{\pi}_{n *} g_{n *} g_{n}^{*} j_{n !} \mu_{l^{r}}
$$

If we apply this to the universal section $\Delta: U \rightarrow E \times{ }_{S} U$ we get the desired result. 
Here is an explicit way to get the extension $\left(\mathcal{P}_{\mathrm{ol}}^{\mathrm{g}}\right)^{\vee}(1)$ from $\left[p_{n *} \mathbb{Z} \rightarrow \pi^{*} P_{H_{n}}\right]$. Consider the exact sequence

$$
0 \rightarrow \pi^{*} P_{H_{n}}\left[l^{r}\right] \rightarrow \pi^{*} P_{H_{n}} \stackrel{l^{r}}{\rightarrow} \pi^{*} P_{H_{n}} \rightarrow \mathbb{Z} / l^{r} \mathbb{Z} \rightarrow 0
$$

defined by the $l^{r}$-multiplication and the map $p_{n *} \mathbb{Z} \rightarrow \pi^{*} P_{H_{n}}$. The pull-back gives an extension

$$
0 \rightarrow \pi^{*} P_{H_{n}}\left[l^{r}\right] \rightarrow \mathcal{E} \rightarrow p_{n *} \mathbb{Z} \rightarrow \mathbb{Z} / l^{r} \mathbb{Z} \rightarrow 0 .
$$

If we tensor this with $\mathbb{Z} / l^{r} \mathbb{Z}$ over $\mathbb{Z}$, then we get an exact sequence

$$
0 \rightarrow \pi^{*} P_{H_{n}}\left[l^{r}\right] \rightarrow \mathcal{E} \otimes_{\mathbb{Z}} \mathbb{Z} / l^{r} \mathbb{Z} \rightarrow p_{n *} \mathbb{Z} / l^{r} \mathbb{Z} \rightarrow \mathbb{Z} / l^{r} \mathbb{Z} \rightarrow 0,
$$

because the kernel of $p_{n *} \mathbb{Z} \rightarrow \mathbb{Z} / l^{r} \mathbb{Z}$ is a free $\mathbb{Z}$-module. With the above theorem we conclude that $\left(\mathcal{P}_{\mathrm{ol}}^{\mathrm{g}}\right)^{\vee}(1) \cong \mathcal{E} \otimes_{\mathbb{Z}} \mathbb{Z} / l^{r} \mathbb{Z}$.

\subsubsection{The class of the geometric polylog}

Recall from section 4.1.1 that the polylog $\left(\mathcal{P}_{\mathrm{ol}}^{\mathrm{g}}\right)^{\vee}(1)$ defines a class in

$$
\operatorname{Ext}_{U}^{1}\left(I_{n, \Lambda_{r}}, \pi_{n}^{*} R^{1} \pi_{n !} \Lambda_{r, U}(1)\right)
$$

where $I_{n, \Lambda_{r}}$ is the kernel of the map $p_{n *} \Lambda_{r, U} \rightarrow \Lambda_{r, U}$. With lemma 4.1 .2 we can write

$$
\operatorname{cl}\left(\left(\mathcal{P}_{\mathrm{ol}}^{\mathrm{g}}\right)^{\vee}(1)\right) \in \operatorname{Ext}_{U}^{1}\left(I_{n, \Lambda_{r}}, P_{H_{n}}\left[l^{r}\right]\right) .
$$

Let $[\mathfrak{a}]$ be an isogeny of $E$ of degree $\mathrm{Na}$ prime to $l$. We can consider the pull-back $[\mathfrak{a}]^{*}\left(\mathcal{P}_{\mathrm{ol}}^{\mathrm{g}}\right)^{\vee}(1)$ on $[\mathfrak{a}]^{-1} U$. Recall from lemma 3.5.1 that we have an isomorphism $p_{n *} \Lambda_{r, U} \cong[\mathfrak{a}]^{*} p_{n *} \Lambda_{r, U}$. Thus $\left.[\mathfrak{a}]^{*}\left(\mathcal{P}_{\mathrm{ol}}^{\mathrm{g}}\right)^{\vee}\right)^{\vee}(1)$ defines a class in

$$
\operatorname{Ext}_{[\mathfrak{a}]^{-1} U}^{1}\left(I_{n, \Lambda_{r}}, P_{H_{n}}\left[l^{r}\right]\right) \text {. }
$$

Similarly consider the $\mathrm{N} \mathfrak{a}$-multiplication on $\left(\mathcal{P}_{\mathrm{ol}}^{\mathrm{g}}\right)_{n}^{\vee}(1)$ restricted to $[\mathfrak{a}]^{-1} U$. This gives also an extension class in the above group. Denote by

$$
\operatorname{cl}\left(\left([\mathfrak{a}]^{*}-\mathrm{N} \mathrm{a}\right)\left(\mathcal{P}_{\mathrm{ol}}^{\mathrm{g}}\right)^{\vee}(1)\right)
$$

the difference of these two classes. Our aim is to compute the specialization of this class. For this we show first, that this class is in the image of

$$
\operatorname{Ext}_{[\mathfrak{a}]^{-1} U}^{1}\left(p_{n *} \Lambda_{r}, P_{H_{n}}\left[l^{r}\right]\right) \rightarrow \operatorname{Ext}_{[\mathfrak{a}]^{-1} U}^{1}\left(I_{n, \Lambda_{r}}, P_{H_{n}}\left[l^{r}\right]\right)
$$


Lemma 4.1.4. The class of $\operatorname{cl}\left(\left([\mathfrak{a}]^{*}-\mathrm{Na}\right)\left(\mathcal{P}_{\mathrm{ol}}^{\mathrm{g}}\right)^{\vee}(1)\right)$ on $[\mathfrak{a}]^{-1} U$ maps to zero under the boundary map

$$
\operatorname{Ext}_{[\mathfrak{a}]^{-1} U}^{1}\left(I_{n, \Lambda_{r}}, P_{H_{n}}\left[l^{r}\right]\right) \rightarrow \operatorname{Ext}_{[\mathfrak{a}]^{-1} U}^{2}\left(\Lambda, P_{H_{n}}\left[l^{r}\right]\right) .
$$

Proof. The sheaf $[\mathfrak{a}]^{*}\left(\mathcal{P}_{\mathrm{ol}}^{\mathrm{g}}\right)^{\vee}(1)$ can be described as the pull-back of the sequence

$$
0 \rightarrow \pi^{*} P_{H_{n}}\left[l^{r}\right] \rightarrow \pi^{*} P_{H_{n}} \stackrel{l^{r}}{\rightarrow} \pi^{*} P_{H_{n}} \rightarrow \mathbb{Z} / l^{r} \mathbb{Z} \rightarrow 0
$$

by the map

$$
[\mathfrak{a}]^{*} \Delta_{n}: p_{n *} \mathbb{Z} \rightarrow[\mathfrak{a}]^{*} p_{n *} \mathbb{Z} \rightarrow[\mathfrak{a}]^{*} \pi^{*} P_{H_{n}} \cong \pi^{*} P_{[\mathfrak{a}]^{-1} H_{n}} \rightarrow \pi^{*} P_{H_{n}},
$$

where the last map is forgetting the trivialization on $[\mathfrak{a}]^{*} H_{n} \backslash H_{n}$. The sheaf $\mathrm{N} \mathrm{a}\left(\mathcal{P}_{\mathrm{ol}}^{\mathrm{g}}\right)^{\vee}(1)$ is the pull-back by the map N a $\Delta_{n}: p_{n *} \mathbb{Z} \rightarrow \pi^{*} P_{H_{n}} \stackrel{\mathrm{Na}}{\longrightarrow} \pi^{*} P_{H_{n}}$ of the same sequence. We have to compute the composition of these maps with $\pi^{*} P_{H_{n}} \rightarrow \mathbb{Z}$. This composition factors in both cases as $p_{n *} \mathbb{Z} \rightarrow \mathbb{Z} \stackrel{\mathrm{Na}}{\longrightarrow} \mathbb{Z}$. Thus, the difference is zero. This implies the claim.

Consider the difference of the two maps in the above proof and denote this by $\left([\mathfrak{a}]^{*}-\mathrm{Na}\right) \Delta_{n}$. By adjunction this defines a map

$$
\left([\mathfrak{a}]^{*}-\mathrm{Na}\right) \Delta_{n}: \mathbb{Z} \rightarrow \pi_{n}^{*} P_{H_{n}},
$$

which by the above proof even factors through $\pi_{n}^{*} P_{H_{n}}^{0}$. The line bundle which is associated with this map is easily described:

Recall from the end of section 4.1 .2 that the line bundle $\mathcal{O}\left(\Delta_{n}\right)$ on $E_{n} \times{ }_{S} U_{n}$ with its canonical trivialization gives a section $\Delta_{n}: \mathbb{Z} \rightarrow \pi_{n}^{*} P_{H_{n}}$. Then the line bundle $[\mathfrak{a}]^{*} \mathcal{O}\left(\Delta_{n}\right) \otimes \mathcal{O}\left(\Delta_{n}\right)^{\otimes-N \mathfrak{a}}$ on $E_{n} \times_{S}[\mathfrak{a}]^{-1} U_{n}$ defines the section $\left([\mathfrak{a}]^{*}-\mathrm{N} \mathfrak{a}\right) \Delta_{n}$.

Denote by $\delta$ the composition

$$
\delta: H^{0}\left([\mathfrak{a}]^{-1} U_{n}, T_{H_{n}}\right) \rightarrow H^{1}\left([\mathfrak{a}]^{-1} U_{n}, T_{H_{n}}\left[l^{r}\right]\right) \rightarrow H^{1}\left([\mathfrak{a}]^{-1} U_{n}, P_{H_{n}}\left[l^{r}\right]\right),
$$

where the first map is the boundary map for the short exact sequence

$$
0 \rightarrow T_{H_{n}}\left[l^{r}\right] \rightarrow T_{H_{n}} \stackrel{\left[l^{r}\right]}{\longrightarrow} T_{H_{n}} \rightarrow 0
$$

Proposition 4.1.5. The section $\left([\mathfrak{a}]^{*}-\mathrm{Na}\right) \Delta_{n}$ factors through $T_{H_{n}}$ and up to sign, there is an equality

$$
\operatorname{cl}\left(\left([\mathfrak{a}]^{*}-\mathrm{N} \mathfrak{a}\right)\left(\mathcal{P}_{\mathrm{ol}}^{\mathrm{g}}\right)^{\vee}(1)\right)= \pm \delta\left(\left([\mathfrak{a}]^{*}-\mathrm{Na}\right) \Delta_{n}\right)
$$

in $H^{1}\left([\mathfrak{a}]^{-1} U_{n}, P_{H_{n}}\left[l^{r}\right]\right)$. 
Proof. The composition of $\left([\mathfrak{a}]^{*}-\mathrm{Na}\right) \Delta_{n}$ with the map $P_{H_{n}}^{0} \rightarrow P_{n}^{0}$ to the Picard scheme is zero, because $[\mathfrak{a}]^{*} \mathcal{O}\left(\Delta_{n}\right) \cong \mathcal{O}\left(\Delta_{n}\right)^{\otimes N \mathfrak{a}}$. This gives the first claim. The extension class $\delta\left(\left([\mathfrak{a}]^{*}-\mathrm{N} \mathfrak{a}\right) \Delta_{n}\right)$ is given by the pull-back of the sequence (11) with the map $\left([\mathfrak{a}]^{*}-\mathrm{Na}\right) \Delta_{n}: \mathbb{Z} \rightarrow \pi_{n}^{*} T_{H_{n}}$. Using the explicit description following the proof of theorem 4.1 .3 proves the result.

\subsection{Computation of the specialization of the polylog}

Let $t: S \rightarrow U$ be an $N$-torsion point. We want to compute the specialization at $t$ of the polylog.

\subsubsection{Specialization of the class of the geometric polylog}

Let $t: S \rightarrow U$ be an $N$-torsion point and $[\mathfrak{a}]: E \rightarrow E$ an isogeny of degree $\mathrm{N} \mathfrak{a}$ relatively prime to $N l$. Let $H_{n, t}:=p_{n}^{-1}(t) \subset U_{n}$ be the inverse image of the $N$-torsion point $t: S \rightarrow U$ in $U_{n}$. To compute the specialization of the polylog following section 3.5 we need to compute the restriction of the class $\left([\mathfrak{a}]^{*}-\mathrm{Na}\right)\left(\mathcal{P}_{0} l_{n}^{\mathrm{g}}\right)^{\vee}(1)$ in $H^{1}\left([\mathfrak{a}]^{-1} U_{n}, T_{H_{n}}\left[l^{r}\right]\right)$ to $H_{n, t}$. We let $t$ : $H_{n, t} \rightarrow[\mathfrak{a}]^{-1} U_{n}$ also be the inclusion of $H_{n, t}$ by abuse of notation. Denote the restriction of the polylog by

$$
\operatorname{cl}\left(t^{*}\left([\mathfrak{a}]^{*}-\mathrm{N} \mathfrak{a}\right)\left(\mathcal{P}_{\mathrm{ol}}^{\mathrm{g}}\right)^{\vee}(1)\right) \in H^{1}\left(H_{n, t}, T_{H_{n}}\left[l^{r}\right]\right) .
$$

We have to compute the section $t^{*}\left([\mathfrak{a}]^{*}-\mathrm{N} \mathrm{a}\right) \Delta_{n}: \mathbb{Z} \rightarrow t^{*} T_{H_{n}}$, which is defined by the trivialization of the (trivial) line bundle $t^{*}[\mathfrak{a}]^{*} \mathcal{O}\left(\Delta_{n}\right) \otimes t^{*} \mathcal{O}\left(\Delta_{n}\right)^{\otimes-\mathrm{N} \mathfrak{a}}$ on $H_{n, t}$.

To describe this section in terms of functions, we extend our base to a Galois covering $S_{n}$ of $S$, where $H_{n, t}$ is rational and then use descent: Let $G_{n}$ be the Galois covering group of $S_{n} / S$. Consider $\prod_{h_{n} \in H_{n}\left(S_{n}\right)} \mathbb{G}_{m}$ and write a typical element of this product as $\sum_{h_{n}} g_{h_{n}}\left(h_{n}\right)$. Similar definitions apply to $\prod_{h_{n} \in H_{n}\left(S_{n}\right)} \mu_{l^{r}}$. Let us write $T_{H_{n}}$ as a quotient:

Lemma 4.2.1. The group $T_{H_{n}}\left(S_{n}\right)$ can be identified as the $G_{n}$-invariant elements of the quotient

$$
\begin{aligned}
\prod_{h_{n} \in H_{n}\left(S_{n}\right)} \mathbb{G}_{m}\left(S_{n}\right) & \rightarrow T_{H_{n}}\left(S_{n}\right) \\
\sum_{h_{n}}\left(g_{h_{n}}\right)\left(h_{n}\right) & \mapsto\left(\frac{g_{h_{n}}}{g_{e_{n}}}\right)\left(h_{n}\right) .
\end{aligned}
$$

Proof. Clear because the character group of $T_{H_{n}}$ is $\operatorname{ker}\left(p_{H_{n} *} \mathbb{Z} \rightarrow \mathbb{Z}\right)$. 
This gives

$$
H^{0}\left(H_{n, t} \times{ }_{S} S_{n}, T_{H_{n}}\right)=\prod_{t_{n} \in H_{n, t}\left(S_{n}\right)} T_{H_{n}}\left(S_{n}\right) .
$$

With this notation we rewrite the section $t^{*}\left([\mathfrak{a}]^{*}-\mathrm{Na}\right) \Delta_{n}: \mathbb{Z} \rightarrow t^{*} T_{H_{n}}$. Note first that $t^{*}[\mathfrak{a}]^{*} \mathcal{O}\left(\Delta_{n}\right)=[\mathfrak{a}]^{*} \mathcal{O}\left([\mathfrak{a}] H_{n, t}\right)$ and $t^{*} \mathcal{O}\left(\Delta_{n}\right)^{\otimes-\mathrm{N} \mathfrak{a}}=\mathcal{O}\left(H_{n, t}\right)^{\otimes-\mathrm{N} \mathfrak{a}}$. The section $t^{*}\left([\mathfrak{a}]^{*}-\mathrm{N} \mathfrak{a}\right) \Delta_{n}: \mathbb{Z} \rightarrow t^{*} T_{H_{n}}$ can now be written as

$$
\sum_{t_{n} \in H_{n, t}\left(S_{n}\right)} s\left(t_{n}\right)
$$

where $s\left(t_{n}\right) \in T_{H_{n}}\left(S_{n}\right)$. This section is given by the trivialization of the line bundle $[\mathfrak{a}]^{*} \mathcal{O}\left([\mathfrak{a}] t_{n}\right) \otimes \mathcal{O}\left(t_{n}\right)^{\otimes-N \mathfrak{a}}$. Using the lemma, we write $s\left(t_{n}\right)=$ $\sum_{h_{n}} s\left(t_{n}\right)_{h_{n}}\left(h_{n}\right)$, with $s\left(t_{n}\right)_{h_{n}} \in \mathbb{G}_{m}$.

We want to compute $s\left(t_{n}\right)_{h_{n}}$ for $h_{n} \in H_{n}\left(S_{n}\right) \backslash e_{n}$. For this we need the elliptic units on $E_{n}$.

\subsubsection{Elliptic units}

Elliptic units were introduced and studied by Robert and Gillard. An algebraic approach to elliptic units was proposed by Kato.

Let us recall the definition of the elliptic units and their characterization in Ka2 III 1.1.5. (see also Scho]).

With Kato let us make the definition that $a, b \in \operatorname{End}_{\mathcal{O}_{S}}(E)$ are relatively prime $(a, b)=1$, if $\operatorname{ker}(a) \cap \operatorname{ker}(b)=e$ where $e$ is endowed with the reduced subscheme structure and $a b=b a$.

Theorem 4.2.2. (see Kag III 1.1.5. and $S \mathrm{Sch}$ thm 1.2.1) Let $a \in \operatorname{End}_{\mathcal{O}_{S}}(E)$ be an endomorphism with $(a, 6)=1$. There is a unique section

$$
\theta_{a} \in \mathcal{O}^{*}(E \backslash \operatorname{ker} a)
$$

compatible with base change in $S$, with the following properties:

i) $\operatorname{Div}\left(\theta_{a}\right)=\operatorname{deg}(a)(e)-\operatorname{ker} a$

ii) for any $b \in \operatorname{End}_{\mathcal{O}_{S}}(E)$ with $(a, b)=1$

$$
b_{*} \theta_{a}=\theta_{a} .
$$

iii) Moreover, for $b \in \operatorname{End}_{\mathcal{O}_{S}}(E)$ with $(6, b)=1$ and $a b=b a$

$$
\frac{\theta_{a^{\circ} b}}{\theta_{a}^{\operatorname{deg}(b)}}=\frac{\theta_{b} \circ a}{\theta_{b}^{\operatorname{deg}(a)}} .
$$

Definition 4.2.3. The values of $\theta_{a}$ at torsion sections $t: \mathcal{O}_{S} \rightarrow E \backslash$ ker $a$ are called elliptic units. 


\subsubsection{The specialization of the polylog in terms of elliptic units}

We turn to the computation of $s\left(t_{n}\right)=\sum_{h_{n}} s\left(t_{n}\right)_{h_{n}}\left(h_{n}\right)$ from the end of section 4.2 .1 .

Proposition 4.2.4. Let $[\mathfrak{a}]$ be relatively prime to $N l$. Then the section $s\left(t_{n}\right)_{h_{n}} \in$ $\mathbb{G}_{m}\left(S_{n}\right)$ is given by

$$
s\left(t_{n}\right)_{h_{n}}=\left(\frac{\theta_{\mathfrak{a}}\left(h_{n}-t_{n}\right)}{\theta_{\mathfrak{a}}\left(-t_{n}\right)}\right)^{-1}
$$

for $h_{n} \in H_{n}\left(S_{n}\right) \backslash e_{n}$ using the identification from lemma 4.2.1.

Proof. The section $s\left(t_{n}\right)_{h_{n}}$ is given by evaluating an isomorphism

$$
[\mathfrak{a}]^{*} \mathcal{O}\left([\mathfrak{a}] t_{n}\right) \cong \mathcal{O}\left(t_{n}\right)^{\otimes N \mathfrak{a}}
$$

at $h_{n}$. Let $T_{-t_{n}}$ be the translation with $-t_{n}$ on $E_{n}$, then

$$
[\mathfrak{a}]^{*} \mathcal{O}\left([\mathfrak{a}] t_{n}\right) \cong T_{-t_{n}}^{*}[\mathfrak{a}]^{*} \mathcal{O}\left(e_{n}\right) \text { and } \mathcal{O}\left(t_{n}\right)^{\otimes N \mathfrak{a}} \cong T_{-t_{n}}^{*} \mathcal{O}\left(e_{n}\right)^{\otimes N \mathfrak{a}}
$$

The function $\theta_{\mathfrak{a}}$ gives a section of $\mathcal{O}\left(e_{n}\right)^{\otimes \mathrm{Na}} \otimes[\mathfrak{a}]^{*} \mathcal{O}\left(e_{n}\right)^{\otimes-1}$ and thus $T_{-t_{n}}^{*} \theta_{\mathfrak{a}}$ gives a section of

$$
\mathcal{O}\left(t_{n}\right)^{\otimes \mathrm{N} \mathfrak{a}} \otimes[\mathfrak{a}]^{*} \mathcal{O}\left([\mathfrak{a}] t_{n}\right)^{\otimes-1}
$$

This proves the claim.

Remark: Note that in this proposition we do not really need the elliptic units, because any function with the right divisor would also describe the sections $s\left(t_{n}\right)$. The point is that there are two more problems to solve before we get the specialization of the elliptic polylog. The first problem is rather trivial. It is the computation of the map $\operatorname{pr}_{t}$ from (8), which is nothing but the identification of $H_{n, t}$ with $H_{n}$ via the projection map. The second problem is that we still have to compute the splitting from 3.5 .8 and it is here that the elliptic units will be necessary because of their norm compatibility property.

Recall the definition of the map $\mathrm{pr}_{t}$ from 8. We write

$$
\begin{aligned}
\operatorname{pr}_{t}: H_{n, t}\left(S_{n}\right) & \rightarrow H_{n}\left(S_{n}\right) \\
t_{n} & \mapsto \widetilde{t}_{n}
\end{aligned}
$$


for the projection of $t_{n}$ to $H_{n}$. Consider the section

$$
\prod_{\widetilde{t}_{n} \in H_{n}\left(S_{n}\right)} \sum_{h_{n}}\left(\frac{\theta_{\mathfrak{a}}\left(h_{n}-t_{n}\right)}{\theta_{\mathfrak{a}}\left(-t_{n}\right)}\right)\left(h_{n}\right)
$$

of $\prod_{\tilde{t}_{n} \in H_{n}\left(S_{n}\right)} T_{H_{n}}\left(S_{n}\right)$. It is invariant under the group $G_{n}$ and thus defines a section in $H^{0}\left(H_{n}, T_{H_{n}}\right)$. Denote by

$$
\delta: H^{0}\left(H_{n}, T_{H_{n}}\right) \rightarrow H^{1}\left(H_{n}, T_{H_{n}}\left[l^{r}\right]\right)
$$

the boundary map. The main result of this section can now be formulated as follows:

Proposition 4.2.5. The class of $\pm \operatorname{pr}_{t} t^{*}\left([\mathfrak{a}]^{*}-\mathrm{Na}\right)\left(\mathcal{P o l}_{n}^{\mathrm{g}}\right)^{\vee}(1)$ is given by

$$
\delta\left(\prod_{\tilde{t}_{n} \in H_{n}\left(S_{n}\right)} \sum_{h_{n}}\left(\frac{\theta_{\mathfrak{a}}\left(h_{n}-t_{n}\right)}{\theta_{\mathfrak{a}}\left(-t_{n}\right)}\right)\left(h_{n}\right)\right) \in H^{1}\left(H_{n}, T_{H_{n}}\left[l^{r}\right]\right),
$$

where $t_{n}$ is an $l^{n} N$-division point.

\subsubsection{The splitting}

In the last section we have computed

$$
\operatorname{cl}\left(\operatorname{pr}_{t} t^{*}\left([\mathfrak{a}]^{*}-\mathrm{Na}\right)\left(\mathcal{P o l}_{n}^{\mathrm{g}}\right)^{\vee}(1)\right) \in H^{1}\left(H_{n}, T_{H_{n}}\left[l^{r}\right]\right) .
$$

Recall that the torus $T_{H_{n}}$ is defined by the character group $I\left[H_{n}\right]:=\operatorname{ker}\left(p_{H_{n} *} \mathbb{Z} \rightarrow\right.$ $\mathbb{Z})$. By definition of $\mathcal{I}_{n}^{\mathrm{g}}$, we get that $I\left[H_{n}\right] \otimes \Lambda=\mathcal{I}_{n}^{\mathrm{g}}$ is the augmentation ideal in $\mathcal{R}_{n}^{\mathrm{g}}$. In particular

$$
T_{H_{n}}\left[l^{r}\right]=\underline{\operatorname{Hom}}_{S}\left(\mathcal{I}_{n}^{\mathrm{g}}, \mu_{l^{r}}\right) .
$$

This gives $H^{1}\left(H_{n}, T_{H_{n}}\left[l^{r}\right]\right)=H^{1}\left(S, \underline{\operatorname{Hom}}_{S}\left(\mathcal{I}_{n}^{\mathrm{g}}, \mathcal{R}_{n}^{\mathrm{g}}(1)\right)\right)$. We have a map (cf. 3.5.6) $\mathcal{R}_{n}^{\mathrm{g}}(1) \rightarrow \underline{\operatorname{Hom}}_{S}\left(\mathcal{I}_{n}^{\mathrm{g}}, \mathcal{R}_{n}^{\mathrm{g}}(1)\right)$ given explicitly as follows: Write $\mathcal{R}_{n}^{\mathrm{g}}(1)=$ $\Lambda(1)\left[H_{n}\right]$ and observe that $\mathcal{I}_{n}^{\mathrm{g}} \subset \Lambda\left[H_{n}\right]$ is generated by $\left(h_{n}\right)-\left(e_{n}\right)$ with $h_{n} \in$ $H_{n}$. Then

$$
\begin{aligned}
\Lambda(1)\left[H_{n}\right] & \rightarrow \underline{\operatorname{Hom}}_{S}\left(\mathcal{I}_{n}^{\mathrm{g}}, \Lambda(1)\left[H_{n}\right]\right) \\
\left(h_{n}\right) & \mapsto\left\{\left(h_{n}^{\prime}\right)-\left(e_{n}\right) \mapsto\left(h^{\prime}{ }_{n}+h_{n}\right)-\left(h_{n}\right)\right\} .
\end{aligned}
$$

This induces a map (cf. corollary 3.5.7)

$$
H^{1}\left(S, \mathcal{R}_{n}^{\mathrm{g}}(1)\right)=H^{1}\left(H_{n}, \mu_{l^{r}}\right) \rightarrow H^{1}\left(H_{n}, T_{H_{n}}\left[l^{r}\right]\right) .
$$

To compute the splitting it suffices to write down a norm compatible element in $H^{1}\left(H_{n}, \mu_{l^{r}}\right)$, which maps to the class of $\pm \operatorname{pr}_{t} t^{*}\left([\mathfrak{a}]^{*}-\mathrm{N} \mathfrak{a}\right)\left(\mathcal{P}_{\mathrm{ol}}^{\mathrm{g}}\right)^{\vee}(1)$ in $H^{1}\left(H_{n}, T_{H_{n}}\left[l^{r}\right]\right)$. Recall that $\widetilde{t}_{n}$ is the projection to $H_{n}$. 
Proposition 4.2.6. The element

$$
\delta \sum_{\widetilde{t}_{n} \in H_{n}\left(S_{n}\right)} \theta_{\mathfrak{a}}\left(-t_{n}\right)\left(\widetilde{t}_{n}\right) \in H^{1}\left(H_{n}, \mu_{l^{r}}\right)
$$

maps under (19) to $\pm \operatorname{cl}\left(\operatorname{pr}_{t} t^{*}\left([\mathfrak{a}]^{*}-\mathrm{Na}\right)\left(\mathcal{P o l}_{n}^{\mathrm{g}}\right)^{\vee}(1)\right)$.

Proof. This is a straightforward computation. The element

$$
\sum_{\widetilde{t}_{n}} \theta_{\mathfrak{a}}\left(-t_{n}\right)\left(\widetilde{t}_{n}\right)
$$

maps to the mapping, which sends $\left(h_{n}\right)-\left(e_{n}\right)$ to

$$
\sum_{\widetilde{t}_{n}} \theta_{\mathfrak{a}}\left(-t_{n}\right)\left(h_{n}+\widetilde{t}_{n}\right)-\sum_{\widetilde{t}_{n}} \theta_{\mathfrak{a}}\left(-t_{n}\right)\left(\widetilde{t}_{n}\right)
$$

The first sum can be rewritten as

$$
\sum_{\widetilde{t}_{n}} \theta_{\mathfrak{a}}\left(h_{n}-t_{n}\right)\left(\widetilde{t}_{n}\right)
$$

so that we get $\sum_{\widetilde{t}_{n}} \frac{\theta_{\mathfrak{a}}\left(h_{n}-t_{n}\right)}{\theta_{\mathfrak{a}}\left(-t_{n}\right)}\left(\widetilde{t}_{n}\right)$. This is the desired result.

We show now that the element defined in proposition 4.2 .6 is norm compatible if we vary $n$. This will imply that we have actually computed the splitting and thus the $l$-adic Eisenstein classes.

Let $\mathrm{N}_{n, n^{\prime}}$ be the norm map from $E_{n}$ to $E_{n^{\prime}}$ for $n \geq n^{\prime} .$.

Proposition 4.2.7. In $H^{1}\left(H_{n^{\prime}}, \mu_{l^{r}}\right)$ the following equality holds:

$$
\mathrm{N}_{n, n^{\prime}} \delta \sum_{\widetilde{t}_{n} \in H_{n}(S)} \theta_{\mathfrak{a}}\left(-t_{n}\right)\left(\widetilde{t}_{n}\right)=\delta \sum_{\widetilde{t}_{n^{\prime}} \in H_{n^{\prime}}(S)} \theta_{\mathfrak{a}}\left(-t_{n^{\prime}}\right)\left(\widetilde{t}_{n^{\prime}}\right)
$$

where $\left[l^{n}\right] t_{n}=t$ and $\left[l^{n-n^{\prime}}\right] t_{n}=t_{n^{\prime}}$.

Proof. From theorem 4.2.2 we know that $\mathrm{N}_{n, n^{\prime}} \theta_{\mathfrak{a}}\left(-t_{n}\right)=\theta_{\mathfrak{a}}\left(-t_{n^{\prime}}\right)$ because $\mathfrak{a}$ is prime to $N l$. This proves the claim.

It is clear that the element $\delta \sum_{\widetilde{t}_{n} \in H_{n}\left(S_{n}\right)} \theta_{\mathfrak{a}}\left(-t_{n}\right)\left(\widetilde{t}_{n}\right)$ is compatible with the reduction map $\Lambda_{r} \rightarrow \Lambda_{r^{\prime}}$ for $r \geq r^{\prime}$. Hence we get an element

$$
\left(\delta \sum_{\widetilde{t}_{n} \in H_{n}\left(S_{n}\right)} \theta_{\mathfrak{a}}\left(-t_{n}\right)\left(\widetilde{t}_{n}\right)\right)_{n} \in H^{1}\left(S, \mathcal{R}_{\mathbb{Z}_{l}}^{\mathrm{g}}(1)\right) .
$$


Recall the map

$$
H^{1}\left(S, \mathcal{R}_{\mathbb{Z}_{l}}^{\mathrm{g}}(1)\right)=\operatorname{Ext}_{S, \mathbb{Z}_{l}}^{1}\left(\mathbb{Z}_{l}, \mathcal{R}_{\mathbb{Z}_{l}}^{\mathrm{g}}(1)\right) \stackrel{a}{\rightarrow} \operatorname{Ext}_{S, \mathcal{R}_{\mathbb{Z}_{l}}^{\mathrm{g}}}^{1}\left(\mathcal{I}_{\mathbb{Z}_{l}}^{\mathrm{g}}, \mathcal{R}_{\mathbb{Z}_{l}}^{\mathrm{g}}(1)\right)
$$

from corollary 3.5.7.

Lemma 4.2.8. The element $\left(\delta \sum_{\widetilde{t}_{n} \in H_{n}\left(S_{n}\right)} \theta_{\mathfrak{a}}\left(-t_{n}\right)\left(\widetilde{t}_{n}\right)\right)_{n}$ maps to the class of $\mathcal{P O l}_{\mathbb{Z}_{l}}^{\mathrm{g}}$ under the map a.

Proof. This follows immediately from the above proposition.

\subsubsection{The main theorem on the specialization of the elliptic polylog}

It remains to compute the moment map to get the $l$-adic Eisenstein classes explicitly.

Theorem 4.2.9. Let $\beta=\sum_{t \in E[N](S) \backslash e} n_{t}(t)$ and $[\mathfrak{a}]: E \rightarrow E$ an isogeny relatively prime to $\mathrm{Nl}$. Then for $k>0$ the l-adic Eisenstein class

$$
\left(\beta^{*}\left([\mathfrak{a}]^{*}-\mathrm{N} \mathrm{a}\right) \mathcal{P}_{\mathrm{ol}_{\mathbb{Q}_{l}}}\right)^{k} \in H^{1}\left(S, \operatorname{Sym}^{k} \mathcal{H}_{\mathbb{Q}_{l}}(1)\right)
$$

is given by

$$
\pm \frac{1}{k !}\left(\delta \sum_{t \in E[N](S) \backslash e} n_{t} \sum_{\left[l^{n}\right] t_{n}=t} \theta_{\mathfrak{a}}\left(-t_{n}\right) \widetilde{t}_{n}^{\otimes k}\right)_{n}
$$

where $\widetilde{t}_{n}$ is the projection of $t_{n}$ to $E\left[l^{n}\right]$.

Proof. The recipe to compute the moment map from lemma 3.5.5 combined with lemma 4.2 .8 gives immediately the result.

\section{Proof of the main theorem}

In this section we will carry out the actual comparison between the space $r_{p}\left(\mathcal{R}_{\psi}\right)$ and the Soulé elements $e_{p}\left(\overline{\mathcal{C}}_{\infty} \otimes T_{p} E(k)\right)$ defined by elliptic units.

\subsection{Comparison with the Soulé elements}

We first transfer the result from 4.2.9 into the setting of 1.2 .2 and then compare these elements with the Soulé map $e_{p}$. 


\subsubsection{The specialization of the elliptic polylog}

The theorem 4.2.9 gives us an explicit description of

$$
\left(\beta^{*}\left([\mathfrak{a}]^{*}-\mathrm{N} \mathfrak{a}\right) \mathcal{P}_{\mathrm{ol}_{\mathbb{Q}_{l}}}\right)^{2 k+1} H^{1}\left(\mathcal{O}_{S}, \operatorname{Sym}^{2 k+1} \mathcal{H}_{\mathbb{Q}_{p}}(1)\right),
$$

which we now translate in the setting of section 1.2.2. Note first that $p=l$ and $S=\mathcal{O}_{S}$ and $\mathcal{H}_{\mathbb{Q}_{p}}=T_{p} E \otimes \mathbb{Q}_{p}$. Let $\mathfrak{a} \in \mathcal{O}_{K}$ be prime to $6 p \mathfrak{f}$. Let $\theta_{\mathfrak{a}}$ be the function defined in 4.2.2. To have shorter formulas we introduce the following notation: Define for $\widetilde{t}_{r} \in E\left[p^{r}\right]$

$$
\gamma\left(\widetilde{t}_{r}\right)^{k}:=<\widetilde{t}_{r}, \sqrt{d_{K}} \widetilde{t}_{r}>^{\otimes k}
$$

where $\left.\left\langle{ }_{-},\right\rangle_{-}\right\rangle$is the Weil pairing and $\sqrt{d_{K}}$ is a root of the discriminant of $K / \mathbb{Q}$. Note that $\gamma\left(\psi(\mathfrak{p}) \widetilde{t}_{r}\right)^{k}=(\mathrm{N} \mathfrak{p})^{k} \beta\left(\widetilde{t}_{r}\right)^{k}$. Recall from the end of section 3.5 that we have an endomorphism $\left([\mathfrak{a}]^{*}-\mathrm{Na}\right)$ of $H^{1}\left(\mathcal{O}_{S}, \operatorname{Sym}^{2 k+1} \mathcal{H}_{\mathbb{Q}_{p}}(1)\right)$, which is multiplication with $\mathfrak{a}^{2 k+1} \mathrm{Na}-\mathrm{Na}$.

Theorem 5.1.1. Let $p \nmid 6 \mathrm{~N} \mathfrak{f}$ and denote for a $p^{r} \mathrm{~N} \mathfrak{f}$-torsion point $t_{r}$ by $\widetilde{t}_{r}$ its projection to $E\left[p^{r}\right]$. Then with $t=\Omega f^{-1}$

$\left([\mathfrak{a}]^{*}-\mathrm{N} \mathfrak{a}\right) r_{p}(\xi)= \pm \frac{\mathrm{N} \mathfrak{f}^{3 k+2} L_{p}(\bar{\psi},-k)^{-1}}{2^{k-1} \psi(f)}\left(\delta \mathrm{N}_{K(\mathfrak{f}) / K} \sum_{p^{r} t_{r}=t} \theta_{\mathfrak{a}}\left(-t_{r}\right) \otimes \widetilde{t}_{r} \otimes \gamma\left(\widetilde{t}_{r}\right)\right)_{r}$

where $\widetilde{t}_{r}$ is the projection of $t_{r}$ to $E\left[p^{r}\right]$.

Proof. We have by definition and and theorem 1.2 .5

$$
\begin{aligned}
r_{p}(\xi) & =\frac{(-1)^{k-1}(2 k+1) ! L_{p}(\bar{\psi},-k)^{-1}}{2^{k-1} \psi(f) \mathrm{N} \mathrm{f} f^{k}} \mathcal{K}_{\mathcal{M} r_{p}}\left(\mathcal{E}_{\mathcal{M}}^{2 k+1}(\beta)\right) \\
& =\frac{(-1)^{k-1}(2 k+1) ! \mathrm{N} \mathrm{f}{ }^{3 k+2} L_{p}(\bar{\psi},-k)^{-1}}{2^{k-1} \psi(f)} \mathcal{K}_{\mathcal{M}}\left(\beta^{*} \mathcal{P}_{\mathrm{ol}_{\mathbb{Q}_{l}}}\right)^{2 k+1} .
\end{aligned}
$$

By definition of $[\mathfrak{a}]^{*} \mathcal{P}_{\mathrm{Ol}_{\mathbb{Q}_{l}}}$ we see immediately, that

$$
\left([\mathfrak{a}]^{*}-\mathrm{N} \mathfrak{a}\right)\left(\beta^{*} \mathcal{P}_{\mathrm{ol}_{\mathbb{Q}_{l}}}\right)^{2 k+1}=\left(\beta^{*}\left([\mathfrak{a}]^{*}-\mathrm{N} \mathfrak{a}\right) \mathcal{P}_{\mathrm{ol}_{\mathbb{Q}_{l}}}\right)^{2 k+1} .
$$

With the above notation, we have

$$
\mathcal{K}_{\mathcal{M}}\left(\widetilde{t}_{r}^{\otimes 2 k+1}\right)=\widetilde{t}_{r} \otimes \gamma\left(\widetilde{t}_{r}\right)^{k}
$$

so that theorem 4.2 .9 gives:

$$
\left([\mathfrak{a}]^{*}-\mathrm{N} \mathfrak{a}\right) r_{p}(\xi)= \pm \frac{\mathrm{N} \mathfrak{f}^{3 k+2} L_{p}(\bar{\psi},-k)^{-1}}{2^{k-1} \psi(f)}\left(\delta \mathrm{N}_{K(\mathfrak{f}) / K} \sum_{p^{r} t_{r}=t} \theta_{\mathfrak{a}}\left(-t_{r}\right) \otimes \widetilde{t}_{r} \otimes \gamma\left(\widetilde{t}_{r}\right)\right)_{r}
$$

This is the desired result. 


\subsubsection{The comparison theorem}

We want to rewrite the formula in theorem 5.1.1 in terms of the norm map for $K_{n}(\mathfrak{f}) / K$.

Fix a prime $\mathfrak{p}$ of $K$ where $E$ has good reduction. Define a uniformizer by $\pi:=\psi(\mathfrak{p})$. Denote by

$$
H_{r, t}^{\mathfrak{p}}:=\left\{t_{r} \in E\left[\mathfrak{p}^{r} \mathfrak{f}\right] \mid \pi^{r} t_{r}=t\right\} .
$$

We write $t_{r}=\left(\widetilde{t_{r}}, \pi^{-r} t\right) \in E\left[\mathfrak{p}^{r} \mathfrak{f}\right]=E\left[\mathfrak{p}^{r}\right] \oplus E[\mathfrak{f}]$. Denote by $K\left(\mathfrak{p}^{r} \mathfrak{f}\right)$ the ray class field for $\mathfrak{p}^{r} \mathfrak{f}$. This is the field where the $E\left[\mathfrak{p}^{r} \mathfrak{f}\right]$-points are rational. Let $\sigma_{\mathfrak{p}}$ be the Frobenius at $\mathfrak{p}$ in the Galois group of $K(\mathfrak{f}) / K$, then $t_{r}=\left(\widetilde{t}_{r}, t^{\sigma_{\mathfrak{p}}^{-r}}\right)$. Recall that $\gamma\left(\widetilde{t}_{r}\right)^{k}:=<\widetilde{t}_{r}, \sqrt{d_{K} t_{r}}>^{\otimes k}$. Define a filtration of $H_{r, t}^{\mathfrak{p}}$ as follows:

$$
F_{r, t}^{i}:=\left\{t_{r}=\left(\widetilde{t_{r}}, \pi^{-r} t\right) \in H_{r, t}^{\mathfrak{p}} \mid \pi^{r-i} \widetilde{t_{r}}=0\right\} .
$$

Thus

$$
H_{r, t}^{\mathfrak{p}}=F_{r, t}^{0} \supset \ldots \supset F_{r, t}^{r}=0 .
$$

Define $T_{\mathfrak{p}} E:=\varliminf_{n} E\left[\mathfrak{p}^{n}\right]$.

Theorem 5.1.2. Let $\mathfrak{p}$ be as above and $t_{r}=\left(\widetilde{t_{r}}, \pi^{-r} t\right) \in F_{r, t}^{0} \backslash F_{r, t}^{1}$. Let $L_{\mathfrak{p}}(\bar{\psi},-k)$ be the Euler factor for $\bar{\psi}$ at $\mathfrak{p}$ evaluated at $-k$, then

$$
\begin{gathered}
L_{\mathfrak{p}}(\bar{\psi},-k)^{-1}\left(\mathrm{~N}_{K(\mathfrak{f}) / K} \sum_{s_{r} \in H_{r, t}^{\mathfrak{p}}} \theta_{\mathfrak{a}}\left(-s_{r}\right) \otimes \widetilde{s}_{r} \otimes \gamma\left(\widetilde{s}_{r}\right)^{k}\right)_{r}= \\
\left(\mathrm{N}_{K\left(\mathfrak{p}^{r} \mathfrak{f}\right) / K}\left(\theta_{\mathfrak{a}}\left(-t_{r}\right) \otimes \widetilde{t}_{r} \otimes \gamma\left(\widetilde{t}_{r}\right)^{k}\right)\right)_{r}
\end{gathered}
$$

in $H^{1}\left(\mathcal{O}_{S}, T_{\mathfrak{p}} E(k+1) \otimes \mathbb{Q}_{p}\right)$ for all $\mathfrak{a}$ relatively prime to $\mathfrak{p f}$.

Proof. Observe that we identified $\operatorname{Hom}_{\mathcal{O}_{p}}\left(T_{p} E, \mathcal{O}_{p}\right) \cong T_{p} E(-1)$ where $T_{p} E$ has now the conjugate linear $\mathcal{O}_{p}$-action. In particular, $\overline{\psi(\mathfrak{p})} t_{r}=t_{r-1}$ for $t_{r} \in E\left[\mathfrak{p}^{r}\right]$. We compute

$$
\begin{aligned}
& \left(\frac{\overline{\psi(\mathfrak{p})}}{\mathrm{Np}^{-k}}\right)^{i} \mathrm{~N}_{K\left(\mathfrak{p}^{r} \mathfrak{f}\right) / K\left(\mathfrak{p}^{r-i} \mathfrak{f}\right)}\left(\theta_{\mathfrak{a}}\left(-t_{r}\right) \otimes \widetilde{t}_{r} \otimes \gamma\left(\widetilde{t}_{r}\right)^{k}\right)= \\
& =\mathrm{N}_{K\left(\mathfrak{p}^{r} \mathfrak{f}\right) / K\left(\mathfrak{p}^{r-i} \mathfrak{f}\right)}\left(\theta_{\mathfrak{a}}\left(-\left(\widetilde{t}_{r}, \pi^{-r} t\right)\right) \otimes \overline{\psi(\mathfrak{p})} \widetilde{t}_{r} \otimes \gamma\left(\overline{\psi(\mathfrak{p})} \widetilde{t}_{r}\right)^{k}\right) \\
& =\left(\mathrm{N}_{K\left(\mathfrak{p}^{r} \mathfrak{f}\right) / K\left(\mathfrak{p}^{r-i} \mathfrak{f}\right)} \theta_{\mathfrak{a}}\left(-\left(\widetilde{t}_{r}, \pi^{-r} t\right)\right)\right) \otimes \widetilde{t}_{r-i} \otimes \gamma\left(\widetilde{t}_{r-i}\right)^{k} \\
& \left.=\theta_{\mathfrak{a}}\left(-\left(\widetilde{t}_{r-i}, \pi^{i-r} t\right)\right)\right) \otimes \widetilde{t}_{r-i} \otimes \gamma\left(\widetilde{t}_{r-i}\right)^{k} \text {, }
\end{aligned}
$$


where we used the distribution relation for $\theta_{\mathfrak{a}}$ (see deSh II 2.5)

$$
\left.\mathrm{N}_{K\left(\mathfrak{p}^{r} \mathfrak{f}\right) / K\left(\mathfrak{p}^{r-i} \mathfrak{f}\right)} \theta_{\mathfrak{a}}\left(-t_{r}\right)=\theta_{\mathfrak{a}}\left(\pi^{i} t_{r}\right)\right)
$$

for the last equality.

As the Galois group of $K\left(\mathfrak{p}^{r-i} \mathfrak{f}\right) / K(\mathfrak{f})$ acts simply transitively on $F_{r, t}^{i} \backslash$ $F_{r, t}^{i+1}$, we get

$$
\begin{aligned}
& \left(\frac{\overline{\psi(\mathfrak{p})}}{\mathrm{Np}^{-k}}\right)^{i} \mathrm{~N}_{K\left(\mathfrak{p}^{r} \mathfrak{f}\right) / K(\mathfrak{f})}\left(\theta_{\mathfrak{a}}\left(-t_{r}\right) \otimes \widetilde{t}_{r} \otimes \gamma\left(\widetilde{t}_{r}\right)^{k}\right)= \\
& \quad \sum_{t_{r-i} \in F_{r, t}^{i} \backslash F_{r, t}^{i+1}} \theta_{\mathfrak{a}}\left(-\left(\widetilde{t}_{r-i}, \pi^{i-r} t\right)\right) \otimes \widetilde{t}_{r-i} \otimes \gamma\left(\widetilde{t}_{r-i}\right)^{k} .
\end{aligned}
$$

We have $\left.\left.\theta_{\mathfrak{a}}\left(-\left(\widetilde{t}_{r-i}, \pi^{i-r} t\right)\right)\right)=\theta_{\mathfrak{a}}\left(-\left(\widetilde{t}_{r-i}, \pi^{-r} t\right)\right)\right)^{\sigma_{\mathfrak{p}}^{i}}$ which gives

$$
\begin{aligned}
\left(\frac{\overline{\psi(\mathfrak{p})}}{\mathrm{Np}^{-k}}\right)^{i} \mathrm{~N}_{K\left(\mathfrak{p}^{r} \mathfrak{f}\right) / K}\left(\theta_{\mathfrak{a}}\left(-t_{r}\right) \otimes \widetilde{t}_{r} \otimes \gamma\left(\widetilde{t}_{r}\right)^{k}\right)= \\
\mathrm{N}_{K(\mathfrak{f}) / K} \sum_{t_{r-i} \in F_{r, t}^{i} \backslash F_{r, t}^{i+1}}\left(\theta_{\mathfrak{a}}\left(-\left(\widetilde{t}_{r-i}, \pi^{-r} t\right)\right) \otimes \widetilde{t}_{r-i} \otimes \gamma\left(\widetilde{t}_{r-i}\right)^{k}\right)
\end{aligned}
$$

because the norm $\mathrm{N}_{K(\mathfrak{f}) / K}$ is the sum over all the Galois translates, which act trivially on $\widetilde{t}_{r-i}$. If we finally take the sum over $i$ and let $r$ get bigger and bigger we get

$$
\begin{aligned}
L_{\mathfrak{p}}(\overline{\psi(\mathfrak{p})},-k) & \left(\mathrm{N}_{K\left(\mathfrak{p}^{r} \mathfrak{f}\right) / K} \theta_{\mathfrak{a}}\left(-t_{r}\right) \otimes \widetilde{t}_{r} \otimes \gamma\left(\widetilde{t}_{r}\right)^{k}\right)_{r}= \\
& \left(\mathrm{N}_{K(\mathfrak{f}) / K} \sum_{t_{r} \in H_{r, t}^{\mathfrak{p}}} \theta_{\mathfrak{a}}\left(-t_{r}\right) \otimes \widetilde{t}_{r} \otimes \gamma\left(\widetilde{t}_{r}\right)^{k}\right)_{r}
\end{aligned}
$$

where we used

$$
\sum_{i \geq 0}\left(\frac{\overline{\psi(\mathfrak{p})}}{N \mathfrak{p}^{-k}}\right)^{i}=\frac{1}{1-\frac{\overline{\psi(\mathfrak{p})}}{N \mathfrak{p}^{-k}}}
$$

This is the desired result.

With theorem 5.1.1 we get: 
Corollary 5.1.3. With the notations of theorem 5.1.1

$$
\left([\mathfrak{a}]^{*}-\mathrm{N} \mathfrak{a}\right) r_{p}(\xi)= \pm \frac{\mathrm{N} \mathfrak{f}^{3 k+2}}{2^{k-1} \psi(f)} \delta\left(\mathrm{N}_{K\left(p^{r} \mathfrak{f}\right) / K} \theta_{\mathfrak{a}}\left(-t_{r}\right) \otimes \widetilde{t}_{r} \otimes \gamma\left(\widetilde{t_{r}}\right)^{k}\right)_{r}
$$

where $p^{r} t_{r}=t$ and $t_{r}$ is a primitive $p^{r} \mathfrak{f}$-torsion point.

Proof. If $p$ is inert of prime this is just a reformulation of theorem 5.1.2. If $p$ is split, $r_{p}$ decomposes into a direct sum for the $\mathfrak{p}$ and the $\mathfrak{p}^{*}$ part. Putting them together gives the result.

\subsection{End of proof of the main theorem}

Here we finich the proof of theorem 1.1.5 by computing the image of the Soulé map $e_{p}$. In the last section we prove that $r_{p}$ is injective on $\mathcal{R}_{\psi}$ if $H_{p}^{2}$ is finite.

\subsubsection{Relation to elliptic units}

Our aim is to show that the elements

$$
\left(\mathrm{N}_{K\left(p^{r} \mathfrak{f}\right) / K} \theta_{\mathfrak{a}}\left(t_{r}\right) \otimes \widetilde{t}_{r} \otimes \gamma\left(\widetilde{t}_{r}\right)^{k}\right)_{r}
$$

where $\mathfrak{a}$ is prime to $6 p \mathfrak{f}$ generate $\left(\overline{\mathcal{C}}_{\infty}^{\chi} \otimes T_{p} E(k)\right)_{\Gamma}$, where $\chi$ is the representation of $\Delta$ on $\operatorname{Hom}_{\mathcal{O}_{p}}\left(T_{p} E(k), \mathcal{O}_{p}\right)$.

Proposition 5.2.1. Let $p \nmid 6 \mathrm{Nf}$ and $\mathfrak{a}$ be an ideal in $\mathcal{O}_{p}$, which is prime to $6 p \mathfrak{f}$ and such that $\mathrm{Na} \not \equiv 1(\bmod p)$. Then the $\mathcal{O}_{p}[[\Gamma]]$-module

$$
\overline{\mathcal{C}}_{\infty}^{\chi} \otimes_{\mathcal{O}_{p}} T_{p} E(k)
$$

is generated by $\left(\theta_{\mathfrak{a}}\left(t_{r}\right) \otimes \widetilde{t}_{r} \otimes \gamma\left(\widetilde{t}_{r}\right)^{k}\right)_{r}$, where $t_{r}$ is a primitive $p^{r} \mathfrak{f}$-division point.

Proof. Let $\mathfrak{b}$ be another ideal prime to $6 p \mathfrak{f}$. Then by theorem 4.2 .2

$$
\begin{aligned}
\left(\sigma_{\mathfrak{a}}-\psi(\mathfrak{a})(\mathrm{N} \mathrm{a})^{k+1}\right)\left(\theta_{\mathfrak{b}}\left(t_{r}\right) \otimes \widetilde{t}_{r} \otimes \gamma\left(\widetilde{t}_{r}\right)^{k}\right) & =\psi(\mathfrak{a})(\mathrm{N} \mathrm{a})^{k}\left(\theta_{\mathfrak{b}}\left(t_{r}\right)^{\sigma_{\mathfrak{a}}-\mathrm{N} \mathfrak{a}} \otimes \widetilde{t}_{r} \otimes \gamma\left(\widetilde{t}_{r}\right)^{k}\right) \\
& =\psi(\mathfrak{a})(\mathrm{N} \mathrm{a})^{k}\left(\theta_{\mathfrak{a}}\left(t_{r}\right)^{\sigma_{\mathfrak{b}}-\mathrm{N} \mathfrak{b}} \otimes \widetilde{t}_{r} \otimes \gamma\left(\widetilde{t}_{r}\right)^{k}\right) .
\end{aligned}
$$

It is enough to show that $\sigma_{\mathfrak{a}}-\psi(\mathfrak{a})(\mathrm{Na})^{k+1}$ is invertible in $\Lambda=\mathcal{O}_{p}[[\Gamma]]$, because $\overline{\mathcal{C}}_{\infty}^{\chi}$ is a torsion free $\Lambda$-module. $\Lambda$ is a local ring if $p$ is inert or prime in $K$ and a product of local rings if $p$ is split. We have $\Lambda / \mathfrak{m}=E[p](k)$, where $\mathfrak{m}$ is either the maximal ideal or the product of the maximal ideals. The element 
$\sigma_{\mathfrak{a}}$ acts via $\psi(\mathfrak{a})(\mathrm{Na})^{k}$ on $E[p](k)$ and thus $\sigma_{\mathfrak{a}}-\psi(\mathfrak{a})(\mathrm{N} \mathrm{a})^{k+1}$ is invertible in $\Lambda$ if $\mathrm{N} \mathfrak{a} \not \equiv 1(\bmod p)$. It remains to see that $\gamma\left(\widetilde{t}_{r}\right)^{k}$ generates $\mathbb{Z}_{p}(k)$. We have

$$
\begin{aligned}
<\widetilde{t}_{r}, \sqrt{d_{K} \widetilde{t}_{r}>^{ \pm 1}} & =\exp \left(p^{-r}|\Omega|^{2}\left(\overline{\sqrt{d_{K}}}-\sqrt{d_{K}}\right)\right) \\
& =\exp \left(-2 i p^{-r}|\Omega|^{2} \sqrt{\left|d_{K}\right|}\right) \\
& =\exp \left(-4 \pi i p^{-r}\right),
\end{aligned}
$$

which is for $p \neq 2$ a primitive root of unity.

Corollary 5.2.2. The image $r_{p}\left(\mathcal{R}_{\psi}\right)$ in $H^{1}\left(\mathcal{O}_{S}, T_{p} E(k+1) \otimes \mathbb{Q}_{p}\right)$ coincides with the image of

$$
e_{p}\left(\left(\overline{\mathcal{C}}_{\infty}^{\chi} \otimes T_{p} E(k)\right)_{\Gamma}\right)
$$

Proof. As $\frac{\mathrm{N}^{3 k+2}}{2^{k-1} \psi(f)}$ is prime to $p$, this follows from corollary 5.1.3 and the definition of $e_{p}$ in 2.2.1.

To conclude the proof of theorem 1.1.5 it remains to see the following lemma:

Lemma 5.2.3. The canonical map

$$
\left(\overline{\mathcal{C}}_{\infty} \otimes T_{p} E(k)\right) \otimes_{\mathcal{O}_{p}[[\mathcal{G}]]}^{\mathbb{L}} \mathcal{O}_{p} \rightarrow\left(\overline{\mathcal{C}}_{\infty} \otimes T_{p} E(k)\right)_{\mathcal{G}} \cong\left(\overline{\mathcal{C}}_{\infty}^{\chi} \otimes T_{p} E(k)\right)_{\Gamma}
$$

is an isomorphism and $\left(\overline{\mathcal{C}}_{\infty}^{\chi} \otimes T_{p} E(k)\right)_{\Gamma} \cong \mathcal{O}_{p}$.

Proof. By [Ru2] theorem 7.7 we have an isomorphism

$$
\overline{\mathcal{C}}_{\infty}^{\chi} \cong \Lambda^{\chi}=\mathcal{O}_{p}[[\Gamma]] .
$$

This implies that the $\mathcal{O}_{p}[[\Gamma]]$-module is induced and hence as $\mathcal{O}_{p}[[\Gamma]]$-module isomorphic to $\mathcal{O}_{p}[[\Gamma]]$. This implies $\left(\overline{\mathcal{C}}_{\infty}^{\chi} \otimes T_{p} E(k)\right)_{\Gamma} \cong \mathcal{O}_{p}$ and the claim of the corollary, because the higher Tor-terms vanish.

We get as a corollary part b) of theorem 1.1.5:

Corollary 5.2.4. The map

$$
\mathcal{R}_{\psi} \otimes \mathbb{Z}_{p} \rightarrow R \Gamma\left(\mathcal{O}_{S}, T_{p} E(k+1) \otimes \mathbb{Q}_{p}\right)[1]
$$

induced by $r_{p}$, gives an isomorphism

$$
\operatorname{det}_{\mathcal{O}_{p}} \mathcal{R}_{\psi} \cong \operatorname{det}_{\mathcal{O}_{p}} R \Gamma\left(\mathcal{O}_{S}, T_{p} E(k+1)\right)^{-1} .
$$

Proof. The complex $\mathcal{R}_{\psi} \otimes \mathbb{Z}_{p} \rightarrow R \Gamma\left(\mathcal{O}_{S}, T_{p} E(k+1) \otimes \mathbb{Q}_{p}\right)[1]$ is isomorphic to

$$
\left(\overline{\mathcal{C}}_{\infty}^{\chi} \otimes T_{p} E(k)\right)_{\Gamma} \stackrel{e_{p}}{\longrightarrow} R \Gamma\left(\mathcal{O}_{S}, T_{p} E(k+1) \otimes \mathbb{Q}_{p}\right)[1]
$$

because by 5.2.2 $r_{p}$ and $e_{p}$ have the same image and as $\mathcal{O}_{p}$-modules $\left(\overline{\mathcal{C}}_{\infty}^{\chi} \otimes\right.$ $\left.T_{p} E(k)\right)_{\Gamma} \cong \mathcal{O}_{p}$ and $\mathcal{R}_{\psi} \otimes \mathbb{Z}_{p} \cong \mathcal{O}_{p}$. Theorem 2.2.11 implies then the claim. 


\subsubsection{Finiteness of $H_{p}^{2}$ and injectivity of $r_{p}$}

Here we prove the addition of theorem 1.1.5, that the finiteness of $H_{p}^{2}$ implies that $r_{p}$ is injective on $\mathcal{R}_{\psi}$.

Recall that by corollary 5.2.2 the image of $r_{p}$ coincides with the image of $e_{p}$. It suffices for the injectivity of $r_{p}$ to prove that $r_{p}\left(\mathcal{R}_{\psi}\right)$ is non zero, because $\mathcal{R}_{\psi} \cong \mathcal{O}_{K}$.

Proposition 5.2.5. Let $H_{p}^{2}$ be finite, then $e_{p}$ is injective.

Proof. We chow first that $H_{p}^{2}$ finite implies the finiteness of $\left(\mathcal{A}_{\infty} \otimes T_{p} E(k)\right)_{\mathcal{G}}$. Note that this is the cokernel of

$$
\left(\mathcal{U}_{\infty} \otimes T_{p} E(k)\right)_{\mathcal{G}} \rightarrow\left(\mathcal{X}_{\infty} \otimes T_{p} E(k)\right)_{\mathcal{G}}
$$

Computing up to finite groups we get from corollary 2.2.7 (using corollary 2.2.10) that this cokernel is isomorphic (up to finite groups) to the cokernel of

$$
H^{1}\left(K \otimes \mathbb{Q}_{p}, E\left[p^{\infty}\right](-k)\right)^{*} \rightarrow H^{1}\left(\mathcal{O}_{S_{p}}, E\left[p^{\infty}\right](-k)\right)^{*}
$$

which is contained in $H^{2}\left(\mathcal{O}_{S_{p}}, T_{p} E(k+1)\right)$. This group is of course finite if $H_{p}^{2}=H^{2}\left(\mathcal{O}_{S}, T_{p} E(k+1)\right)$ is finite. Thus $\left(\mathcal{A}_{\infty} \otimes T_{p} E(k)\right)_{\mathcal{G}}$ is finite. Using lemma 6.2. from [Ru2] we see that this implies that $\left(\mathcal{A}_{\infty} \otimes T_{p} E(k)\right)^{\mathcal{G}}$ is finite. We will now show that this last group controls the kernel of $e_{p}$. It suffices to show that the kernel of $e_{p}$ on $\left(\overline{\mathcal{E}}_{\infty} \otimes T_{p} E(k)\right)_{\mathcal{G}}$ is finite because by Ru2 7.8. both $\overline{\mathcal{E}}_{\infty}$ and $\overline{\mathcal{C}}_{\infty}$ are $\Lambda$-modules of rank 1 with $\overline{\mathcal{E}}_{\infty} / \overline{\mathcal{C}}_{\infty}$ a torsion module. So suppose that the image of $\left(\overline{\mathcal{E}}_{\infty} \otimes T_{p} E(k)\right)_{\mathcal{G}}$ under $e_{p}$ has not rank 1, i.e. is finite. Then, because $\left(\mathcal{U}_{\infty} \otimes T_{p} E(k)\right)_{\mathcal{G}} \cong H^{1}\left(K \otimes \mathbb{Q}_{p}, E\left[p^{\infty}\right](-k)\right)^{*}$ the image of $\left(\overline{\mathcal{E}}_{\infty} \otimes T_{p} E(k)\right)_{\mathcal{G}}$ in $\left(\mathcal{U}_{\infty} \otimes T_{p} E(k)\right)_{\mathcal{G}}$ must be finite as well. The kernel of the map

$$
\left(\overline{\mathcal{E}}_{\infty} \otimes T_{p} E(k)\right)_{\mathcal{G}} \rightarrow\left(\mathcal{U}_{\infty} \otimes T_{p} E(k)\right)_{\mathcal{G}}
$$

is $H_{1}\left(\mathcal{G}, \mathcal{U}_{\infty} / \overline{\mathcal{E}}_{\infty} \otimes T_{p} E(k)\right)$ (group homology). On the other hand, up to finite groups, corollary 2.2.7 implies that $H_{1}\left(\mathcal{G}, \mathcal{X}_{\infty} \otimes T_{p} E(k)\right) \cong H^{2}\left(\mathcal{O}_{S_{p}}, E\left[p^{\infty}\right](-k)\right)^{*}$. By lemma 2.2.8 we get a commutative diagram (up to finite groups)

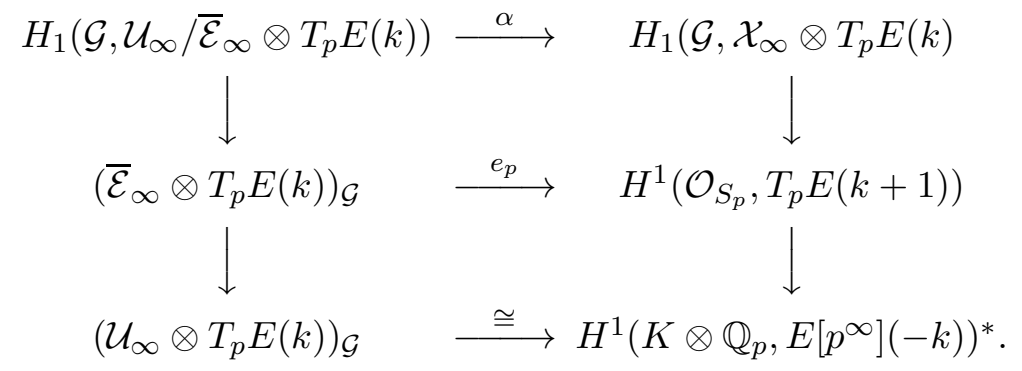


The kernel of the map $\alpha$ is a quotient of $\left(\mathcal{A}_{\infty} \otimes T_{p} E(k)\right)^{\mathcal{G}}$ which by the above is finite. Thus, we arrive at a contradiction and $e_{p}$ can not be zero on the free part of $\left(\overline{\mathcal{E}}_{\infty} \otimes T_{p} E(k)\right)_{\mathcal{G}}$. Hence, $e_{p}$ is non zero on $\left(\overline{\mathcal{C}}_{\infty} \otimes T_{p} E(k)\right)_{\mathcal{G}}$.

\section{References}

[Ar-Ma] M. Artin, B. Mazur: Etale Homotopy, Lecture Notes in Mathematics 100, Springer 1986

[Be1] A. Beilinson: Higher regulators and values of L-functions. J. Sov. Math. 30, 2036-2070 (1985)

[Be2] A. Beilinson: Higher regulators of modular curves, Contemporary Math. Vol. 55 I (1986)

[Be3] A. Beilinson: Polylogarithm and cyclotomic elements, manuscript, no date.

[Be-Le] A. Beilinson, A. Levin: The elliptic polylogarithm, in: U. Jannsen et al.(eds.): Motives, Proceedings Seattle 1991, Providence, RI: American Mathematical Society, Proc. Symp. Pure Math. 55, Pt. 2, 123-190 (1994)

[Bour] N. Bourbaki: Groupes et Algebres des Lie, Hermann, (1972)

[Bl-Ka] S. Bloch, K. Kato: L-functions and Tamagawa numbers of motives, in: P. Cartier et al. eds.: The Grothendieck Festschrift Vol. I, Birkhäuser (1990)

[Del1] P. Deligne: Théorie de Hodge III, Publ. Math. IHES, 5-77 (1974)

[Del2] P. Deligne: Le groupe fondamental de la droite projective moins trois points. in: Ihara et al. (eds.): Galois groups over $\mathbb{Q}$, MSRI Publication (1989)

[Den1] C. Deninger: Higher regulators and Hecke L-series of imaginary quadratic fields I. Invent. math. $96,1-69$ (1989)

[Den2] C. Deninger: Extensions of motives associated to symmetric powers of elliptic curves and to Hecke characters of imaginary quadratic fields, in: F.Catanese (ed.): Arithmetic Geometry, Cortona 1994. 
[deSh] E. deShalit: Iwasawa Theory of Elliptic Curves with Complex Multiplication, Perspectives in Mathematics vol. 3, Academic Press (1987)

[Hu-Ki1] A. Huber, G. Kings: Dirichlet motives via modular curves, Ann. Sci. ENS, 32, 313-345 (1999)

[Hu-Ki2] A. Huber, G. Kings: Degeneration of $l$-adic Eisenstein classes and of the elliptic poylog, Invent. math. 135, 545-594 (1999)

$[\mathrm{HuW} \quad$ A. Huber, J. Wildeshaus: Classical motivic polylogarithm according to Beilinson and Deligne, Doc. Math. J. DMV 3 , 27-133 (1998)

[Ja1] U. Jannsen: Continous étale cohomology, Math. Ann. 280, 207$245(1988)$

[Ja2] U. Jannsen: On the $l$-adic cohomology of varieties over number fields and its Galois cohomology, in: Ihara et al. (eds.): Galois groups over $\mathbb{Q}$, MSRI Publication (1989)

[Ka1] K. Kato: Lectures on the approach to Iwasawa theory for HasseWeil L-functions via $B_{d R}$, in: J.-L. Colliot-Thélène et al.: Arithmetic Algebraic Geometry, LNM 1553, Springer (1993)

[Ka2] K. Kato: Iwasawa theory and p-adic Hodge theory, Kodai math. J. 16 1-31 (1993)

[Ki] G. Kings: K-theory elements for the polylogarithm of abelian schemes, J. reine angew. Math. 517, 103-116 (1999)

[Kn-Mu $\quad$ F. Knudsen, D. Mumford: The projectivity of the moduli space of stable curves I: Preliminaries on "det" and "Div", Math. Scand. 39, 19-55 (1976)

[Ra] M. Raynaud: Spècialisation du Foncteur de Picard, Publ. Math. IHES, 38 27-76 (1970)

[Ru1] K. Rubin: Tate Shafarevich groups and L-functions of elliptic curves with complex multiplication, Invent. math. 89, 527-560 (1987)

[Ru2] K. Rubin: The "main conjectures" of Iwasawa theory for imaginary quadratic fields, Invent. math. 103, 25-68 (1991) 
[Ru3] K. Rubin: Elliptic curves with complex multiplication and the conjecture of Birch and Swinnerton-Dyer, in: Arithmetic Theory of Elliptic Curves, Lecture Notes in Mathematics 1716, Springer 1999

[Scho] A.J. Scholl: An introduction to Kato's Euler system, in: A.J. Scholl, R.L. Taylor (eds.): Galois representations in Arithmetic Algebraic Geometry, Cambridge University Press (1998)

[Sch1] P. Schneider: Über gewisse Galoiscohomologiegruppen, Math. Z. 168 (1979)

[Sch2] P. Schneider: Introduction to the Beilinson conjectures. in: M. Rapoport et al.: Beilinson's conjectures on special values of $L$ functions. Academic Press (1988)

[Se] J.-P. Serre: Groupes algébriques et corps des classes, Hermann (1959)

[Si] J.H. Silverman: Advanced Topics in the Arithmetic of Elliptic Curves, Graduate Texts in Math. vol. 151, Springer (1994)

[So1] C. Soulé: The rank of étale cohomology of varieties over $p$-adic or number fields, Comp. Math. 53, 113-131 (1984)

[So2] C. Soulé: p-adic K-theory of elliptic curves, Duke math. Jour. 54, 249-269 (1987)

[Wi] J. Wildeshaus: Realizations of Polylogarithms, Lecture Notes in Mathematics 1650, Springer 1997.

[Win] K. Wingberg: On the étale K-theory of an elliptic curve with complex multiplication for regular primes, Canad. Math. Bull. 33, 145150 (1990)

[EGA II] A. Grothendieck: Éléments de Géométrie Algébrique II, Publ. Math. IHES, 8 (1961)

[SGA4,III] Séminaire de Géométrie Algébrique 4, Théorie des topos et cohomologie étale des schémas, Springer LNM 305 (1972)

[SGA41/2] Séminaire de Géométrie Algébrique $4 \frac{1}{2}$, Cohomologie étale, Springer LNM 569 (1977) 


\section{Guido Kings}

Mathematisches Institut

Westfälische Wilhelms-Universität Münster

Einsteinstr. 62

48149 Münster

Germany

e-mail: kings@math.uni-muenster.de 